\title{
STEREO IMPACT Investigation Goals, Measurements, and Data Products Overview
}

\author{
J.G. Luhmann · D.W. Curtis · P. Schroeder · J. McCauley • R.P. Lin · D.E. Larson • \\ S.D. Bale · J.-A. Sauvaud - C. Aoustin - R.A. Mewaldt - A.C. Cummings · E.C. Stone • \\ A.J. Davis · W.R. Cook • B. Kecman • M.E. Wiedenbeck • T. von Rosenvinge • \\ M.H. Acuna - L.S. Reichenthal · S. Shuman - K.A. Wortman • D.V. Reames • \\ R. Mueller-Mellin • H. Kunow • G.M. Mason • P. Walpole • A. Korth • T.R. Sanderson • \\ C.T. Russell • J.T. Gosling
}

Received: 17 October 2006 / Accepted: 8 March 2007 /

Published online: 1 June 2007

(C) Springer Science+Business Media, Inc. 2007

\begin{abstract}
The IMPACT (In situ Measurements of Particles And CME Transients) investigation on the STEREO mission was designed and developed to provide multipoint solar wind and suprathermal electron, interplanetary magnetic field, and solar energetic particle information required to unravel the nature of coronal mass ejections and their heliospheric consequences. IMPACT consists of seven individual sensors which are packaged into a boom suite, and a SEP suite. This review summarizes the science objectives of IMPACT, the instruments that comprise the IMPACT investigation, the accommodation of IMPACT on the STEREO twin spacecraft, and the overall data products that will flow from the IMPACT measurements. Accompanying papers in this volume of Space Science Reviews highlight the individual sensor technical details and capabilities, STEREO project plans for the use of IMPACT data, and modeling activities for IMPACT (and other STEREO) data interpretation.
\end{abstract}

Keywords STEREO · Coronal mass ejection · Solar energetic particles · Suprathermal electrons · Solar wind electrons · Interplanetary magnetic field · Multipoint heliospheric measurements

J.G. Luhmann $(\varangle)$ - D.W. Curtis · P. Schroeder · J. McCauley · R.P. Lin · D.E. Larson · S.D. Bale Space Sciences Laboratory, University of California, Berkeley, CA 94720, USA e-mail: jgluhman@ssl.berkeley.edu

J.-A. Sauvaud · C. Aoustin

CESR, BP 4346, 31028 Toulouse Cédex 4, France

R.A. Mewaldt · A.C. Cummings · E.C. Stone · A.J. Davis · W.R. Cook · B. Kecman

California Institute of Technology, Pasadena, CA 91125, USA

M.E. Wiedenbeck

Jet Propulsion Laboratory, Pasadena, CA 91109, USA

T. von Rosenvinge · M.H. Acuna - L.S. Reichenthal - S. Shuman · K.A. Wortman · D.V. Reames NASA/Goddard Space Flight Center, Greenbelt, MD 20771, USA 


\section{List of acronyms}

$\begin{array}{ll}\text { ACR } & \text { Anomalous Cosmic Rays } \\ \text { CIR } & \text { Corotating Interaction Region } \\ \text { CME } & \text { Coronal Mass Ejection } \\ \text { GCR } & \text { Galactic Cosmic Rays } \\ \text { ICME } & \text { Interplanetary Coronal Mass Ejection } \\ \text { IDPU } & \text { Instrument Data Processing Unit } \\ \text { IMF } & \text { Interplanetary Magnetic Field } \\ \text { HET } & \text { High Energy Telescope } \\ \text { LET } & \text { Low Energy Telescope } \\ \text { MAG } & \text { Magnetometer } \\ \text { SEP } & \text { Solar Energetic Particles } \\ \text { SEPT } & \text { Solar Electron and Proton Telescope } \\ \text { SIT } & \text { Suprathermal Ion Telescope } \\ \text { STE } & \text { Suprathermal Electron Telescope } \\ \text { STEREO } & \text { Solar Terrestrial Observatory } \\ \text { SWEA } & \text { Solar Wind Electron Analyzer }\end{array}$

\section{Introduction to the IMPACT Investigation}

The primary mission of the STEREO (Solar TERrestrial RElations Observatory) twin spacecraft is to understand the 3-dimensional nature of the Sun's corona, including the eruptions known as coronal mass ejections (CMEs), from their birth in the corona to their impact at the Earth. The IMPACT (In situ Measurements of Particles and CME Transients) investigation focuses on the invisible particles and fields that affect Earth's space weather by contributing solar wind and suprathermal electron, interplanetary magnetic field, and solar energetic particle (SEP) measurements toward this goal. IMPACT measurements are also essential for other STEREO objectives, especially for relating the 3D corona and solar wind structure, for advancing knowledge of the sources and physics controlling solar energetic particle acceleration and propagation, and for unraveling the relationship between CMEs

R. Mueller-Mellin · H. Kunow
University of Kiel, 24118 Kiel, Germany

G.M. Mason

Applied Physics Laboratory, Johns Hopkins University, Laurel, MD 20723, USA

P. Walpole

University of Maryland, College Park, MD 20742, USA

A. Korth

Max-Planck-Institut für Sonnensystemforschung, Lindau, Germany

T.R. Sanderson

Research and Scientific Support Division, ESA, Noorwijk, The Netherlands

C.T. Russell

Institute of Geophysics and Planetary Physics, UCLA, Los Angeles, CA 90095, USA

J.T. Gosling

Laboratory for Atmospheric and Space Physics, University of Colorado, Boulder, CO 80309, USA

Springer 
and the solar magnetic cycle (see the overall STEREO mission goals described by Kaiser et al., this volume, 2007).

IMPACT includes seven scientifically related in situ sensors on each spacecraft, a telescoping boom, and an instrument data processing unit that provides the primary interface between the sensors and the spacecraft. Detailed descriptions of the different IMPACT sensors can be found in companion papers by Mewaldt et al. (this volume, 2007), MuellerMellin et al. (this volume, 2007), von Rosenvinge et al. (this volume, 2007), Mason et al. (this volume, 2007), Acuna et al. (this volume, 2007), Sauvaud et al. (this volume, 2007), and Lin et al. (this volume, 2007), and the novel boom design is described by Ullrich et al. (this volume, 2007). A previous brief summary of the complete IMPACT investigation can be found in Luhmann et al. (2005). In this paper we provide more details about the overall makeup and science goals of IMPACT including its data plans and products. The IMPACT team is committed to the regular integration of IMPACT data with the other STEREO observations to take full advantage of the information obtained by the observatories. Complementary PLASTIC investigation ion composition measurements, and SWAVES plasma wave measurements are described in accompanying papers by Galvin et al. and Bougeret et al., respectively. Joint observing and data analysis plans involve these other in situ measurements, as well as the SECCHI investigation imaging (Howard et al., this volume, 2007) and SWAVES radio remote sensing results (Bougeret et al., this volume, 2007).

The IMPACT investigation was designed to fulfill many of the in situ measurement requirements for STEREO, illustrated by Fig. 1. The strength and orientation of the in-

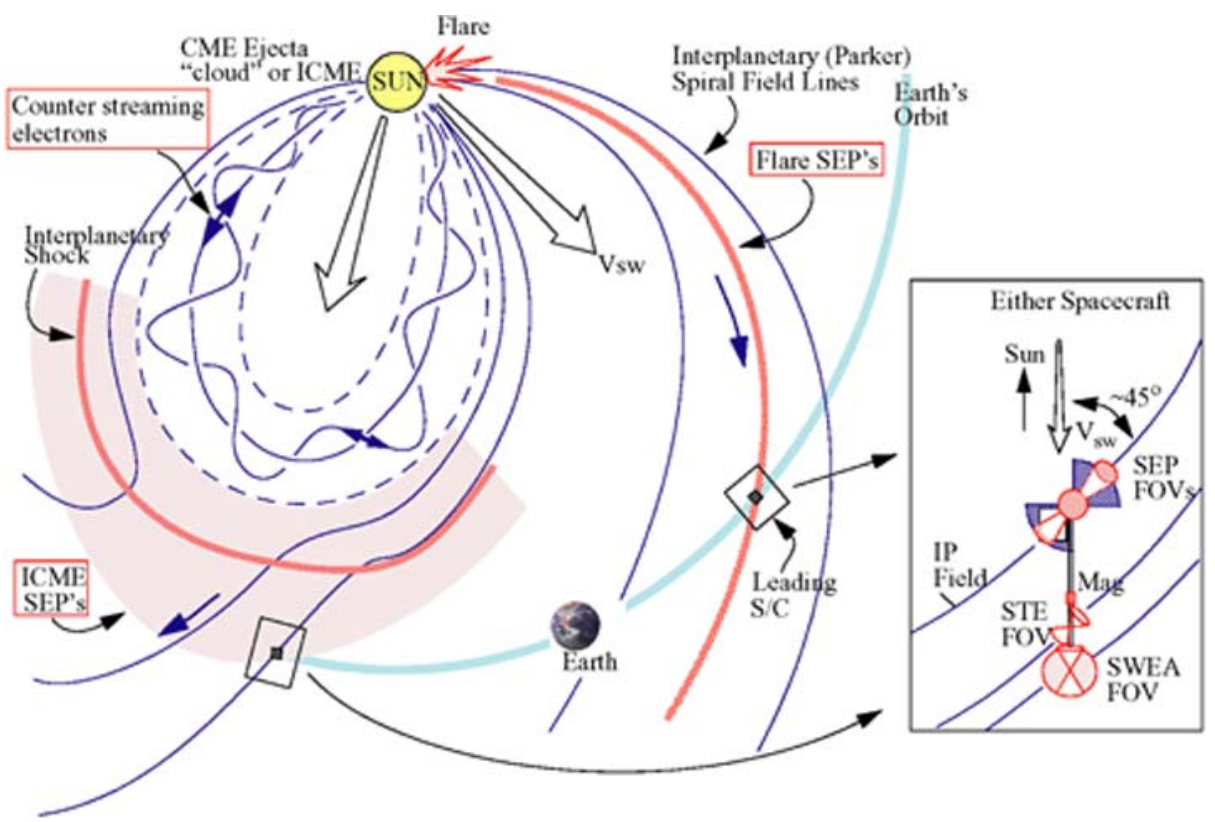

Fig. 1 Illustration of the interaction of a flux rope-like interplanetary CME (ICME) structure inferred from pre-STEREO measurements with the ambient solar wind. Counterstreaming suprathermal electron beams within the ejecta have been used to deduce its structure and size. An interplanetary shock is formed when the ejected mass of plasma and field moves sufficiently fast with respect to the ambient solar wind ahead. This shock often is a source of solar energetic particles that race ahead of it along the spiral interplanetary field lines to arrive at $1 \mathrm{AU}$ well before the ICME and shock. The inset shows how the instruments of the IMPACT investigation on STEREO, described later in the text, are configured to make in situ measurements of the structures and particle populations in this picture at each spacecraft location 
terplanetary magnetic field at the two different STEREO spacecraft, combined with the suprathermal (several $100 \mathrm{~s}$ of $\mathrm{eV}$ ) electron measurements help determine whether the local magnetic fields of the interplanetary CME, the ICME, are rooted at the Sun at one or both ends or disconnected. Such magnetic topology information tells us about the coronal origins of the CME/ICME and also about the role of processes such as reconnection at the Sun and in the interplanetary medium. Higher energy electron, and also ion, detectors provide information on whether the local field connects to a flaring active region. Detection of SEP events at the two locations, with high time resolution and directional information over the energy range from $10 \mathrm{~s}$ of $\mathrm{keV}$ to $\sim 100 \mathrm{MeV}$, allow remote sensing of the CME-initiated shock location and strength as it travels into the heliosphere. SEP ion composition measurements make it possible to distinguish between electron and heavy ion-rich flare-accelerated particles and solar wind particles accelerated at the interplanetary shock. The lowest energy SEP ions also provide information on the ambient suprathermal ion "seed" populations that may feed the higher energy SEP production at the CME related shocks. With the radio and plasma waves investigation, opportunities exist for in situ plasma microphysics diagnostics on local shock structures and waves, as well as comparisons of radio remote sensing and SEP remote sensing of coronal shocks. The in situ data integration with images and ground based images and magnetograph observations through Sun-to-Earth modeling (e.g. Aschwanden et al., this volume, 2006) enables a STEREO era of regular cradle-to-grave analyses of the CME phenomenon (e.g. Fox et al. 1998; Webb et al. 2000). In particular, details of the conditions on the Sun that lead to specific 1 AU consequences can be investigated, and used to refine observational strategies for future space weather missions such as Solar Dynamics Observatory, SDO, and the Heliospheric Sentinels, and for formulating updated science questions for Solar Orbiter and Solar Probe.

Most solar wind structures can be categorized as either corotating or transient, with examples being long-lived high-speed streams and ICMEs, respectively. In both cases, identical measurements of the same structures at the two STEREO locations flanking the Earth test ideas about their size and uniformity and help characterize the features causing magnetic storms and SEP events at $1 \mathrm{AU}$. The spacecraft separation increases at $\sim 45 \mathrm{deg} / \mathrm{year}$, providing the opportunity to watch the two sets of in situ observations increasingly differ. Invaluable additions to STEREO in such multipoint studies are the corresponding in situ observations upstream of Earth on the ACE and WIND spacecraft, creating regular third-point measurements of conditions at 1 AU with separation rates (from STEREO spacecraft) of $\sim 22 \mathrm{deg} /$ year. The nominal angular width of ICMEs has been inferred to be $\sim 90^{\circ}$ although shocks preceding their ejecta "drivers" may produce much wider $\left(\sim 180^{\circ}\right)$ disturbances at 1 AU. At the end of the second year of STEREO heliocentric orbit observations, the Ahead and Behind spacecraft will be $\sim 90^{\circ}$ apart. Single STEREO spacecraft ICME detections will become the norm, with ACE and WIND extending the period of possible multipoint ICME measurements. Quadrature observations, in which one spacecraft measures the 1 AU disturbances from CMEs observed on the limb of the Sun by the SECCHI coronagraphs on the other spacecraft, will then become the primary mode of measurement. In particular, the SECCHI Heliospheric Imager (HI) is expected to track ICME density perturbations to the Earth, where they can be detected by the $\mathrm{L} 1$ in situ instruments. The quadrature observations are the most valuable for investigating how particular events and structures imaged near the Sun evolve into the plasma and field structures observed at 1 AU. They will also test the concept of a space weather forecasting system located at a heliographic longitude removed from the Sun-Earth line.

As part of the STEREO Space Weather Beacon, IMPACT supplies real-time plasma electron, magnetic field, and SEP key parameters analogous to those currently available 
from the ACE RTSW system (Garrard et al. 1998; Zwickl et al. 1998), with corresponding plasma ion quantities from PLASTIC (see Galvin et al., this volume, 2007). ACE real time solar wind plasma, magnetic field, and SEP data are currently being used to drive space weather models that forecast the Earth's radiation environment, ionospheric dynamics, and general geomagnetic activity levels $\sim 30-45$ min ahead of their occurrence (see http://www.srl.caltech.edu/ACE/ASC/related_sites.html). Because STEREO Beacon measurements come from two spacecraft flanking the Earth at increasing distances, their potential applications differ from those using an L1 monitor. A challenge for forecasters is how to take advantage of these data sets. In this respect, STEREO complements efforts within NASA's Living With a Star program whose goal is a time-dependent, predictive global model of the solar wind.

The ultimate success of IMPACT in its role on STEREO depends on obtaining comprehensive observations of a sufficient number of CME-related events to allow multipoint structural diagnosis, and when the spacecraft are near quadrature ( $\sim 90$ degrees apart), to allow detailed comparisons between coronal and heliospheric imager images and in situ measurements. The timing of the two-year STEREO prime mission near solar activity minimum is advantageous for minimizing the ambiguity in identifying solar cause and interplanetary effect, but also reduces the number of events that will be detected in situ. Only one or two clearly flux rope-like ICME ejecta per month were observed on the WIND spacecraft during a similar period between cycles 22 and 23. Continuous operation is necessary to collect several events that are well-sampled and simple enough in structure to constrain complete Sun-to-Earth CME event interpretations and reach the STEREO primary mission goal. Continuation of mission operations into the next solar maximum in $\sim 2010$ can greatly increase the number and variety of observed events available for analysis.

The success of both IMPACT and STEREO also depends on dedicated modeling activities (see Achwanden et al., this volume) that can describe the physical and geometrical connections between what is seen in both the SECCHI images and the in situ measurements. Correlative data analysis by itself generally cannot provide the complex and coupled 3D descriptions available from state-of-the-art realistic simulations of the global corona, solar wind, CMEs, ICMEs and SEPs. The SAIC 3D MHD corona model coupled with the University of Colorado/SEC solar wind model (Riley et al. 2003; Odstrcil et al. 2002), and the University of Michigan 3D MHD adaptive grid solar wind and ICME transport model (Manchester et al. 2005; Roussev et al. 2003), will be brought to bear on the interpretation of IMPACT observations. Overlap between the IMPACT and other STEREO modeling efforts enables the treatment of the CME problem in its entirety.

This overview of IMPACT begins with brief descriptions of specific science issues targeted by the investigation. We next describe the instruments that provide the measurements needed to address them. We conclude with a summary of the data and data analysis plans. The latter provides the potential user of IMPACT data with a guide to products, tools, and access information.

\section{IMPACT Science Objectives}

\subsection{Reconstructing 3D Pictures of CMEs and Their Interplanetary Consequences}

\subsubsection{Coronal Connections}

Years of CME observations with coronagraphs on Skylab, Solwind, SMM and SOHOLASCO, as well as with ground-based coronagraphs, have yielded no generally accepted 
paradigm for CME generation. Nonetheless, this substantial observational background provides the starting point for STEREO. Key solar imaging results include the observed association of CMEs with the coronal helmet streamer belt (Hundhausen 1993), their solar cycle dependence (Webb and Howard 1994), their frequent 3-part (core, cavity, loop) structure on the limb and association with filament or prominence activations or disruptions (Webb et al. 2000), their apparent temporal and spatial coincidence with soft X-ray sigmoidal structures and post-eruption arcades (Canfield et al. 1999), and waves propagating across the solar surface observed in the EUV (Thompson et al. 2000). Another important observation from coronagraphs is the distribution of apparent CME speeds, which peaks at velocities well below the average solar wind velocity, but extends up to over 1,000 km/s (Gosling et al. 1976; Hundhausen et al. 1994). A few events observed by SOHO LASCO appeared to be traveling at speeds $\sim 2500 \mathrm{~km} / \mathrm{s}$ (Yashiro et al. 2005). What causes such a range of CME speeds is unknown, but it is clear that the speed of a CME has important heliospheric and geomagnetic consequences.

The ICME signatures measured by IMPACT and PLASTIC provide a test of the canonical pre-STEREO picture in Fig. 1. Figure 2 shows an example of in situ plasma, field, and suprathermal electron measurements from the WIND spacecraft during the passage of an isolated ICME (from Larson et al. 1997). Shocks form ahead of the faster ICMEs so that the disturbance associated with a fast ICME generally consists of a leading shock, a compressed ambient solar wind sheath, and the ICME itself that is energized in the eruption. Flux rope models have been a standard way of interpreting the magnetic fields of ICMEs, though they only fit $\sim 30 \%$ of the observations at $1 \mathrm{AU}$ (Gosling 1990). The essential question of what feature or structure in the corona actually becomes the ICME (e.g., Kumar and Rust 1996; Leamon et al. 2004) is one of the major issues STEREO hopes to resolve. Some ejecta can be fit with flux ropes that have leading edge field orientations consistent with the prevailing large scale coronal streamer belt field orientation, and inferred axes roughly parallel to the heliospheric current sheet (Mulligan et al. 1998; Li et al. 2001) as suggested by Fig. 3a. The solar magnetic cycle dependence of the inferred flux rope polarity, defined by the leading northward or southward magnetic fields in the ejecta, is illustrated in Fig. 3b (from Li and Luhmann 2004). This striking evidence of the ejecta relationship to the large scale coronal magnetic fields and the solar dynamo is not yet understood. It appears that the handedness of the flux rope field rotations can often be related to the local photospheric field geometry around CME-associated erupting filaments (Bothmer and Schwenn 1998; Kumar and Rust 1996). It thus appears that both small scale fields and the overlying large scale coronal fields may contribute to ICME topology.

Bidirectional or counterstreaming suprathermal electron anisotropies within many ICME ejecta are commonly interpreted (e.g., Gosling et al. 1987) as meaning at least partial connection of ICME fields to the Sun at both ends (see Fig. 1) - a picture consistent with the expanding loop structures in coronagraph images (e.g., Crooker et al. 1993). But ICMEs also often contain unidirectionally streaming electrons (e.g., Shodhan et al. 2000), suggesting that 3-dimensional reconnection within the magnetic legs of CMEs alters their magnetic topology as they propagate out from the Sun (Gosling et al. 1995a). The fact that ICME ejecta often do not fit the flux rope model leaves open the question of whether the flux rope is sometimes not intercepted, or simply does not describe most ejecta field structures. Energetic particle flux anisotropies and high charge state Fe ions occasionally indicate the involvement of flaring active regions near the solar-connected foot-points of the ICME field lines or in the surrounding corona (Kahler and Reames 1991; Larson et al. 1997; Popecki et al. 2000), a feature that may help to identify ICME source regions in STEREO data. In fact major, high-speed ICMEs tend to be preceded by a combination of a flare or 


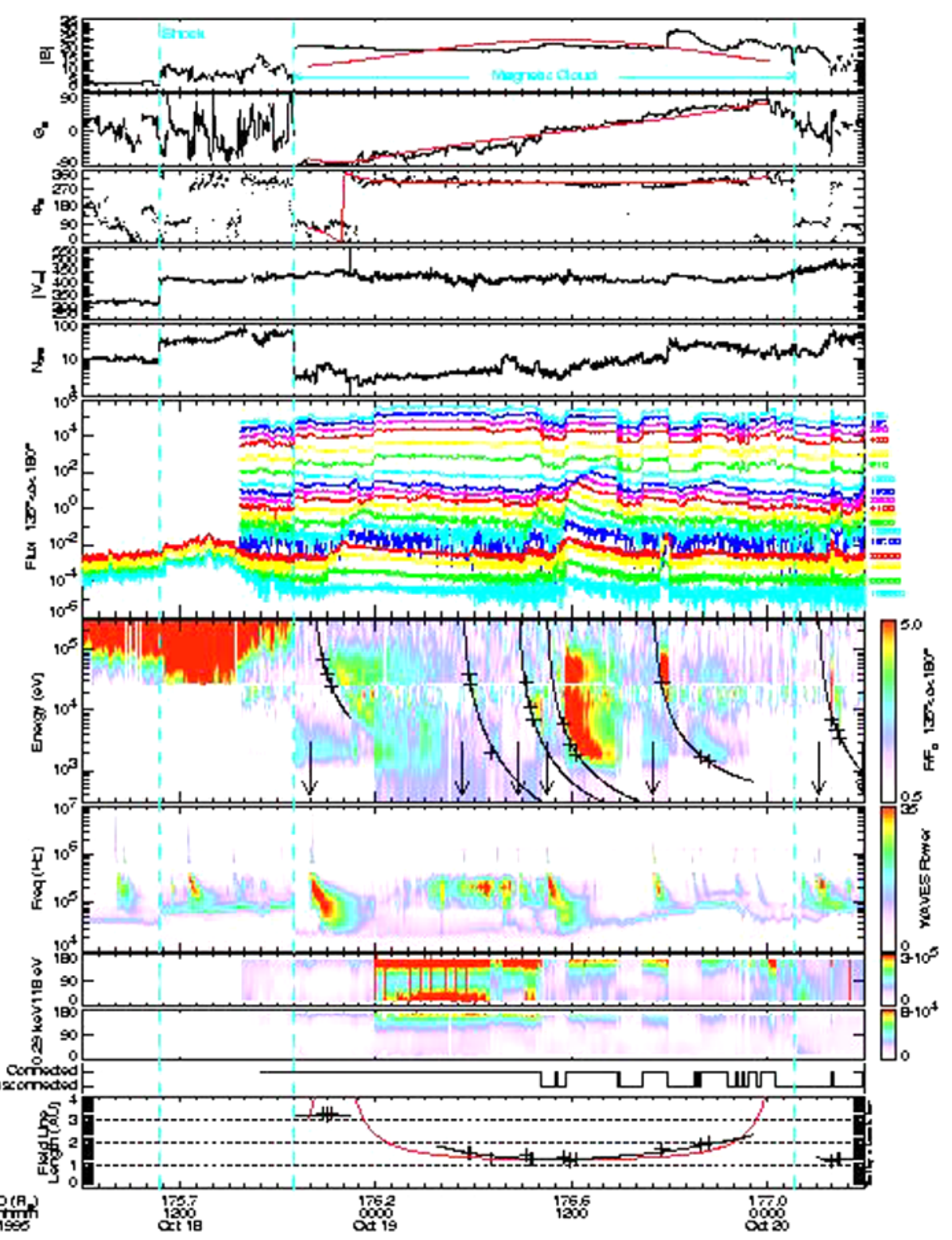

Fig. 2 Example of in situ plasma and field measurements for an isolated ICME observed on WIND by Larson et al. (1997). The top three panels show magnetic field magnitude and direction, with the red line showing a fit to the data of a standard flux rope model. The next panels show solar wind speed and density, and the fluxes of suprathermal electrons of various energies. An electron energy spectrogram, radio spectrogram, and two electron angular spectrograms for suprathermal electrons follow. At the bottom is the field line length inferred from the time of arrival of electrons of various energies, and the curve expected from the flux rope fit at the top

flares and a CME at the Sun (Feynman and Hundhausen 1994), perhaps an indication of the complex coronal conditions that can give rise to those events. 


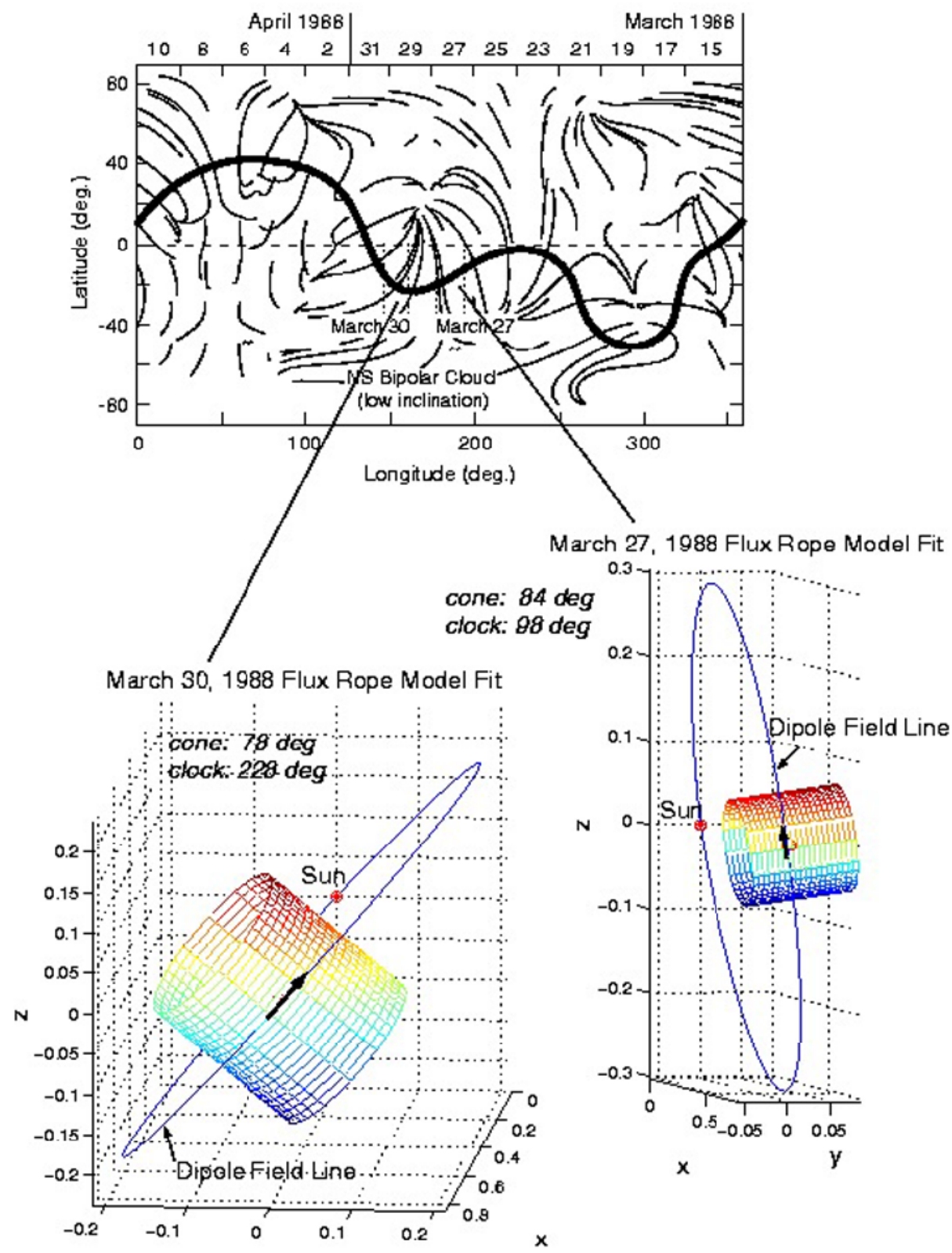

(a)

Fig. 3 a Illustration of the relationship between the coronal streamer belt magnetic field and inferred ICME flux rope ejecta fields (Mulligan 1999, personal communication). b Figure from Li and Luhmann (2004) showing the updated analysis of ICME flux rope polarities compared to the solar cycle and global solar magnetic field. "MC" stands for "magnetic cloud", and "NS" and "SN" for north-south and south-north bipolar signatures in the out-of-ecliptic field component. The gray part of the histogram is derived from PVO measurements, obtained at $0.73 \mathrm{AU}$ 


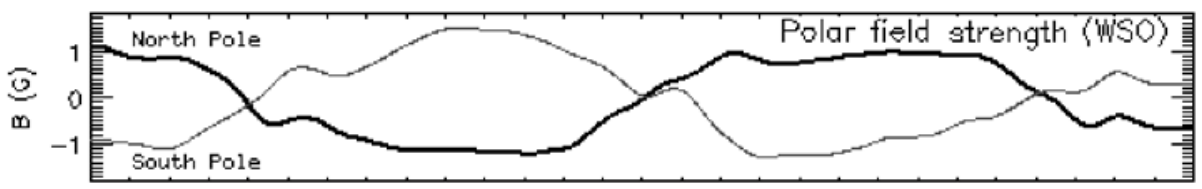

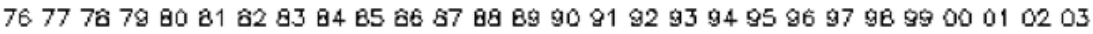
Year

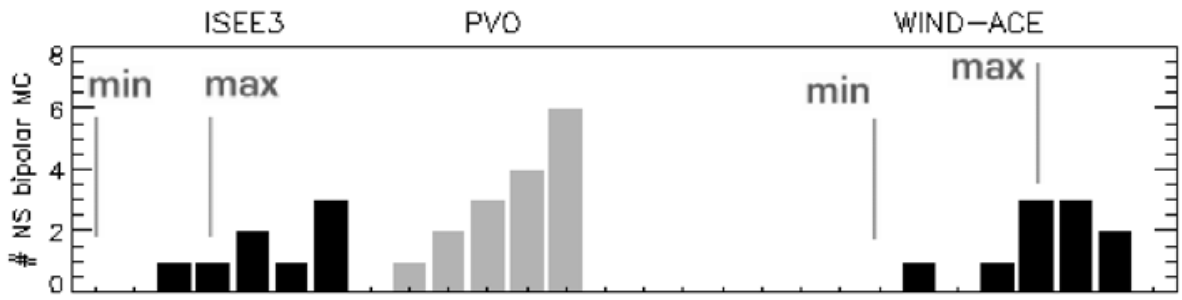

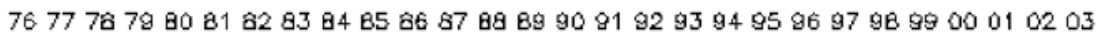

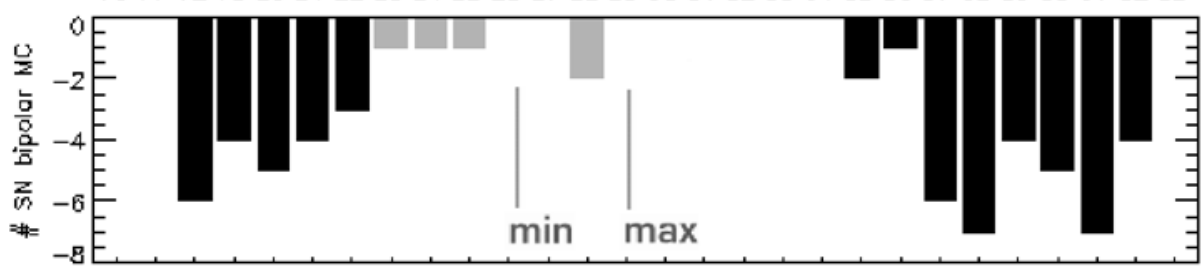

Sunspot Number

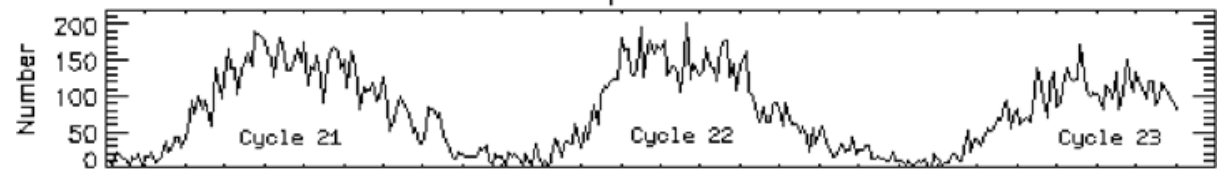

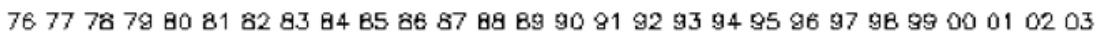

Year

(b)

Fig. 3 (Continued)

Other provocative findings have come from groundbased observations and models. In particular, certain flux emergence patterns in the photosphere have been linked to filament disappearances, and by inference, to CMEs (Feynman and Martin 1995). The solar cycle variability of $\mathrm{CME}$ occurrence resembles the rate of increase of open solar flux at the source surface of potential coronal field models based on photospheric magnetograms (Luhmann et al. 1998). These results complement what is known from the observations described above, especially in their suggestion that CMEs are linked to the evolution of the large scale coronal magnetic field (Harrison et al. 1990). They also pave the way for STEREO investigations of the influence of active region emergence on CME genesis during the rise of cycle 24.

IMPACT observations can constrain our interpretations of CME initiation mechanisms through a number of ICME magnetic field topology diagnostic measurements. In particular, the energy range and wide angular coverage of IMPACT electron measurements allow the suprathermal electron anisotropy measurements that indicate local field connections to the Sun, including connections to active regions. The magnetic field measurements allow single or multipoint flux rope or other topological modeling of the ICME fields for comparisons with the electron diagnostics and structures observed in the SECCHI images. The IMPACT 
SEP measurements help identify the CME source region when energetic particles from either the ICME shock or an associated flare are detected on interplanetary field lines connected to that region. Moreover, SEP ion composition time profiles and fluxes can be used to analyze the CME-flare relationship (e.g., by assuming Fe ion-rich intervals within the larger event are a signature of flare contributions). In all of these cases the availability of multiple site measurements, including any corresponding parameters observed on ACE and WIND, allow us to better infer the location in the corona where the CME initiation occurs.

IMPACT investigation team modelers have shown that it is possible to simulate the formation and eruption of large flux-ropes in the corona in several ways. One approach (Manchester et al. 2005) embeds pre-defined out-of-equilibrium structures in coronal helmet streamers, which then undergo energetic expansion into the heliosphere. Others produce flux rope-like eruptions by energizing existing coronal structures via footpoint shearing followed by canceling or reducing the magnetic flux at the solar boundary (Linker and Mikic 1995). These structures and others from different initiation schemes can be realistically transported to 1 AU using solar wind simulation codes (e.g. Odstrcil et al. 2002; Riley et al. 1997, 2003; Roussev et al. 2003), and then compared with the in situ measurements from IMPACT and PLASTIC. Only comprehensive, sophisticated analyses of this kind can definitively test how ICMEs from various CME generation mechanisms differ, and which, if any, have been confirmed by STEREO observations.

\subsubsection{Interplanetary Consequences}

Not since the days of the Helios spacecraft and the SOLWIND coronagraph on P78-1 have observational comparisons of coronagraph images of CMEs observed on the limb with in situ measurements of ICMEs been regularly possible. These observations (Sheeley et al. 1985) as well as those obtained using Pioneer Venus Orbiter in situ measurements (Lindsay et al. 1999a) showed that ejecta moving slower than the ambient solar wind are accelerated up to the solar wind speed; while ejecta moving faster than the ambient solar wind are decelerated. This result, illustrated in Fig. 4, is supported by the statistics of coronagraph-derived CME speeds and in situ measured ICME speeds (Gosling 1997a). Ulysses measurements at high heliographic latitudes showed that ICMEs in the high-speed polar coronal hole flows

Fig. 4 Lindsay et al. (1999a) result from a quadrature study of CME speeds versus ICME speeds. Other investigations have also derived similar results showing that ICME speeds increase when they are slower than the solar wind, and decelerate when they are faster. Here the open circles represent the highest confidence cases

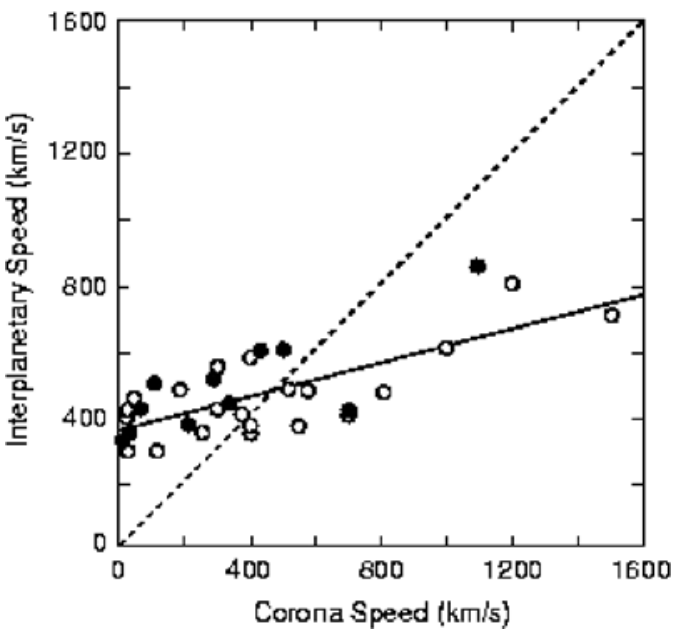


typically have speeds comparable to that of the ambient solar wind speed, but also highlighted the role of expansion in ICME evolution (Gosling et al. 1994a, 1994b; Gosling and Riley 1996). Comparisons between observations of the same ICME at high and low heliographic latitudes illustrate that expansion tends to dominate the ICME interaction with the solar wind at high latitudes, while at lower latitudes relative bulk speed tends to dominate the interaction (Gosling et al. 1995b). Upstream of Earth, ICMEs typically show aspects of both expansion and compression, depending on their location within the ambient solar wind stream structure. They are often found at either the leading or trailing edge of a highspeed stream, consistent with the CME connection with the helmet streamer belt and with the frequent ICME association with the heliospheric current sheet (Crooker et al. 1998).

The interpretation of the in situ observations of ICMEs including their preceding shocks, solar wind compressions or sheaths, and ejecta drivers, is not necessarily straight-forward even if a fairly simple magnetic flux rope structure ejecta is involved. Figure 2 showed an example of a well-studied event from the WIND spacecraft including electron and magnetic field measurements similar to those that IMPACT will obtain. The problem is that what reaches $1 \mathrm{AU}$ is a combination of what is ejected from the Sun and how the related disturbance evolves in transit in the structured solar wind. In the simplest cases the sheath region ahead of a fast ICME is characterized by increased plasma speed, density, temperature, and magnetic field strength that may or may not be preceded by a shock. The ambient magnetic field within the region of compression is reoriented and drapes about the ejecta as illustrated in Fig. 1 (e.g., Hundhausen 1972; Gosling and McComas 1987). The ICME slows as a result of the interaction, while the ambient wind accelerates. If the ICME originally moves faster than the trailing solar wind as well, a rarefaction forms behind the disturbance. In such cases pressure gradients associated with the rarefaction cause both an expansion and a deceleration of the rear portion of the ICME (e.g., Gosling 1996). Compressions from behind by a trailing high speed solar wind stream are also evident in some events, as are compositional signs that a filament of originally colder, denser coronal material forms a part of the observed ICME structures (e.g., Schwenn et al. 1980; Gosling et al. 1980; Skoug et al. 1999). On the other hand, many ICMEs are characterized by ions of high charges states, indicating probable flare origins to some ICME material (e.g., Bame et al. 1979; Lepri et al. 2001).

Because the ambient solar wind is far from homogeneous, particularly at low heliographic latitudes, ICMEs and the shocks they drive must become highly distorted as they propagate outward through the heliosphere (e.g., Riley et al. 1997; Odstrcil and Pizzo 1999a, 1999b, 1999c). It is thus somewhat surprising that about a third of observed ICMEs have magnetic field structures that can locally be fit to simple flux rope models (e.g. Lepping et al. 1990). It is likely that our simplistic cartoons of ICMEs between the Sun and Earth (e.g., Fig. 1) are inadequate. Sophisticated numerical modeling of individual CME initiation and transport cases is required to make substantial progress, as noted above. The combination of 3D reconstructions of the ejections from SECCHI images, and the multipoint perspective on both the ICMEs and surrounding solar wind structure provided by STEREO IMPACT electron and magnetic field measurements and PLASTIC ion measurements, will allow realistic modeling of their complex interplanetary evolution.

A challenge related to the above questions is how best to identify the coronal ejecta portion of ICME disturbances using in situ observations. A variety of signatures (anomalously low plasma ion temperatures, counterstreaming suprathermal electrons, magnetic field rotations, low proton beta, unusually low-variance magnetic fields, anomalous compositions, energetic particle counterstreaming or flux decreases, some of which can be seen in Fig. 2), have proven useful for identifying the ejected material in the solar 
wind, but that identification remains more of an art than a science (e.g., Gosling 1990; Neugebauer and Goldstein 1997). These various signatures often poorly overlap within ejecta, varying from one event to the next or even within a given ICME. For example, the counterstreaming suprathermal electrons in Fig. 2 occur sporadically within the time interval when the smoothly varying magnetic field of the inferred flux rope is observed, suggesting a mixture of open and closed field lines in that event. Recent 2-point magnetometer measurements using NEAR and WIND suggest that the flux rope signature can vary significantly for longitudinal separations as small as 15 degrees (Mulligan and Russell, 2001). The two-point measurements provided by STEREO using identical IMPACT measurements with increasing separation can reveal the spatial extent of various signatures, including the flux rope-like magnetic topology and electron anisotropies, within ejecta passing over both spacecraft. Combined with PLASTIC plasma ion measurements, IMPACT measurements will provide improved ways to characterize ejecta and their boundaries.

STEREO CME/ICME shock tracking and diagnosis (of radial speed profile, strength, extent, structure) from the Sun to 1 AU depends on IMPACT multipoint suprathermal and energetic electron measurements together with SWAVES radio remote sensing data. The directionality and timing of the shock-accelerated electrons serve as probes of the shock surface as it travels Earthward. Analysis of WIND observations using this technique suggests the surface of an ICME-driven shock can be highly corrugated, giving rise to multiple radio emission sites (Bale et al. 1999), Anticipated stereoscopic sounding of shocks using IMPACT electron measurements with SWAVES radio data adds the possibility of obtaining multidimensional information about the shock structures and particle populations corresponding to Type II sources. The IMPACT SEP data and SWAVES observations each provide diagnostics of the radial profile of the ICME shock strength, and in particular a measure of the shock strength in the corona, where it is inferred to be its strongest during major events. The comparison of inferred shock properties at widely separated sites is of particular value because only part of the shock is expected to be a strong SEP source. SWAVES observations can identify that location, filling the gaps between places on the shock for which SEP remote sensing is available.

Many questions related to $\mathrm{CME} / \mathrm{ICME}$ evolution in the solar wind are closely connected to the questions of CME origins. While most CMEs appear to expand as they propagate out through the heliosphere, there is not yet a consensus on what drives these expansions (e.g., Osherovich and Burlaga 1997; Gosling 1997a). Like ion temperatures, the electron temperatures in ICMEs can be extremely cold (Montgomery et al. 1974; Larson et al. 2000), implying that some ICMEs expand adiabatically. Other issues relate to the role of reconnection in creating the magnetic topologies observed within ICMEs near 1 AU. Does the flux rope topology in some ICMEs result from the solar source directly (e.g., Gibson and Low 1998), or is it created in the corona by reconnection during and after CME lift off (e.g., Gosling 1990; Antiochos et al. 1999)? How does reconnection produce the various mixtures of closed, open, and (sometimes) disconnected magnetic topologies inferred to be present within ICMEs based on suprathermal electron anisotropy measurements (e.g., Gosling et al. 1995a; Shodhan et al. 2000)?

As mentioned earlier, some ICMEs move faster than the highest speed solar wind. How are such extreme speeds (up to $\sim 2500 \mathrm{~km} / \mathrm{s}$ ) produced? In general, how does the dynamic interaction of an ICME with the surrounding solar wind affect its observed speed at $1 \mathrm{AU}$ (e.g., Cargill et al. 1996; Gosling and Riley 1996)? Is the flare and/or filament association of CMEs simply incidental or is there a causal relationship and, if so, exactly what is that relationship? How much solar magnetic flux and helicity does the typical ICME contain? What is the origin of observed magnetic polarity changes of ICMEs, in which 
the sequence of the field rotation changes from north-south to south-north or the reverse with the advance of the solar cycle (e.g., Bothmer and Rust 1997; Mulligan et al. 1998; Li and Luhmann 2004)? These and other questions about ICME nature and evolution opened by earlier observations of CMEs and ICMEs, can be addressed anew with STEREO and IMPACT multipoint observations of electron anisotropies and magnetic fields together with interpretive modeling. For example, the increasingly possible numerical simulation of realistic coronal eruptions, propagated through a realistically structured solar wind, can be compared to both the CME images and the IMPACT in situ plasma and field measurements. If they compare favorably, one can probe the geometries and force balances in the simulation results in detail to more fully understand what determines the speed and geometry of the structure that STEREO observed.

\subsection{The Relationship of the 3D Corona to Solar Wind Structure}

The dichotomous high speed and low speed nature of the solar wind was made especially clear by Ulysses in its polar orbit about the Sun (Phillips et al. 1995; McComas et al. 2003). While the high speed wind connection to large polar coronal holes is relatively wellestablished (e.g., Krieger et al. 1973; Neugebauer et al. 1998), the nature of the slow wind remains under debate. Much of the slow solar wind appears to originate from either the cusps of closed field regions making up the coronal streamers, or the edges of coronal holes. This origin accounts for its association with the heliospheric current sheet. The slow solar wind has a highly variable density and ion composition near Earth (e.g., Gosling 1997b) that may result from spatial inhomogeneities or temporal variability in its source regions. The slow solar wind also has different characteristic suprathermal electron anisotropies than the fast solar wind, including less intense heat flux electron beams (Pilipp et al. 1987; Ogilvie et al. 1999), and sometimes sunward directed anisotropies that indicate magnetic fields folded back toward the Sun (Kahler et al. 1984) as well as heat flux dropouts (McComas et al. 1989). One suggestion is that the slow wind at least in part consists of transient events from magnetic reconnection at the coronal hole boundaries or the cusps of streamers (e.g., Crooker and Intriligator 1996; Wang et al. 1998). The bright blobs observed by SOHOLASCO emanating from coronal streamers (Sheeley et al. 1997) may be visible evidence of this process, but they have proven difficult to identify with in situ measurements. The twin STEREO spacecraft offer the possibility of relating features seen by the coronagraph on one spacecraft with structure in the slow solar wind detected on the other by IMPACT. IMPACT electron measurements, far from the contaminating influence of Earth's bow shock, also provide especially good opportunities for definitive analyses of the slow solar wind magnetic field topology.

During the STEREO mission, knowledge of the prevailing ambient 3-dimensional coronal and solar wind structure is needed for providing the context of both CME origins in the corona and their propagation in the solar wind. IMPACT's continuous 2-point measurements of the solar wind plasma and magnetic field, together with the imaging data, will allow the use of coupled corona/solar wind 3D models (e.g. Aschwanden et al., this volume, 2006) to infer global from local or multipoint behavior. It is expected that the L1 monitors ACE and WIND will continue to operate throughout the STEREO mission, in which case 3 or 4-point measurements are routinely available for analysis. Moreover, the Mercury-bound MESSENGER spacecraft is in its cruise phase, Venus Express is in orbit at Venus, and MGS and Mars Express are orbiting Mars, giving the possibility of providing in situ particle and field information over a wide heliolongitude and radial range. Ulysses provides special opportunities for out-of-ecliptic sampling, for more distant quadrature observations, and for coalignments 
allowing observations of the radial evolution of structures. Taking advantage of these other missions makes an effective inner heliosphere constellation for further constraining the 3D solar wind structure deduced from STEREO in situ observations.

\subsection{SEP Acceleration and Propagation}

Major solar energetic particle events (SEPs) usually are closely associated with CMEs and are a key STEREO measurement objective. The duration and intensity of these large gradual events are in contrast to the impulsive, generally weaker, flare-generated events, an example of which is shown next to a gradual event example in Fig. 5. Peak fluxes of the particles in these events are strongly correlated with maximum CME speeds close to the Sun (Kahler et al. 1984). By comparing height-time profiles of CMEs with particle intensities at 1 AU, Kahler (1994) inferred that acceleration peaks when the shock is at 5-10 solar radii for protons with energies up to $\sim 21 \mathrm{GeV}$. However, acceleration of protons and other ions to $\sim 1 \mathrm{GeV}$ sometimes continues out to $1 \mathrm{AU}$ and beyond (Reames 1999). Many gradual SEP events have a local maximum in intensity near shock passage. Such intensifications are known as ESP (Energetic Storm Particle) events. Sometimes the ESP increase at shock passage contains the largest flux measured in a SEP event. These intensifications are thought to result from shock-accelerated particles trapped by the shock and related upstream and downstream field fluctuations. The earlier, more anisotropic parts of gradual events are produced by leakage from this region, the leaked particles racing well ahead of the shock source. The relationship between the ESP events and the prompt parts of the gradual events are still a subject of research, with particle anisotropies (e.g. Reames 1999) and shock-associated waves (e.g. Sanderson et al. 1985) an important element of the data interpretation. Major SEP events are observed in most years of the 11-year solar cycle, except near solar minimum (e.g., Shea and Smart 2002).

Investigations of the heliolongitude extent of SEP events with respect to their source locations indicate they can span over 180 degrees (Cane et al. 1988). However, the intensity-time
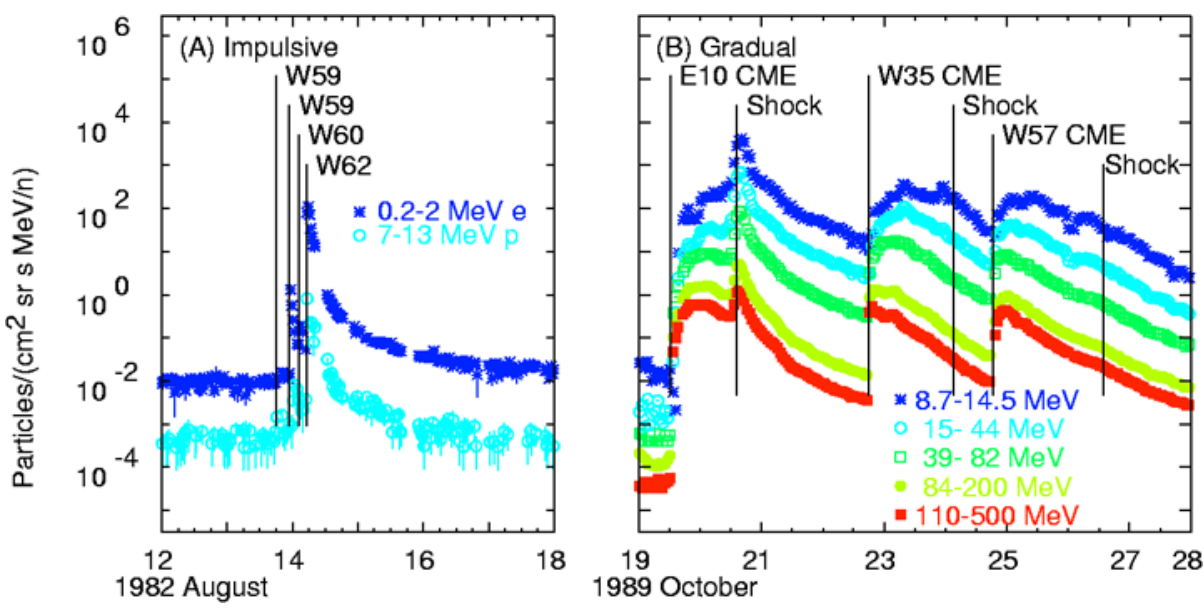

Fig. 5 Illustrations of impulsive (flare-generated) and gradual (interplanetary shock-generated) SEP events observed on the WIND spacecraft. The flare and CME times and locations on the solar disk with respect to the central meridian are indicated in these time series. The impulsive event on the left lasts several hours while the gradual events, here a sequence of three, last several days each. Note the shock arrival ESP event in the first gradual event in near coincidence with shock passage 


\section{Locate ICME and/or Flare Acceleration Site(s) of SEP}

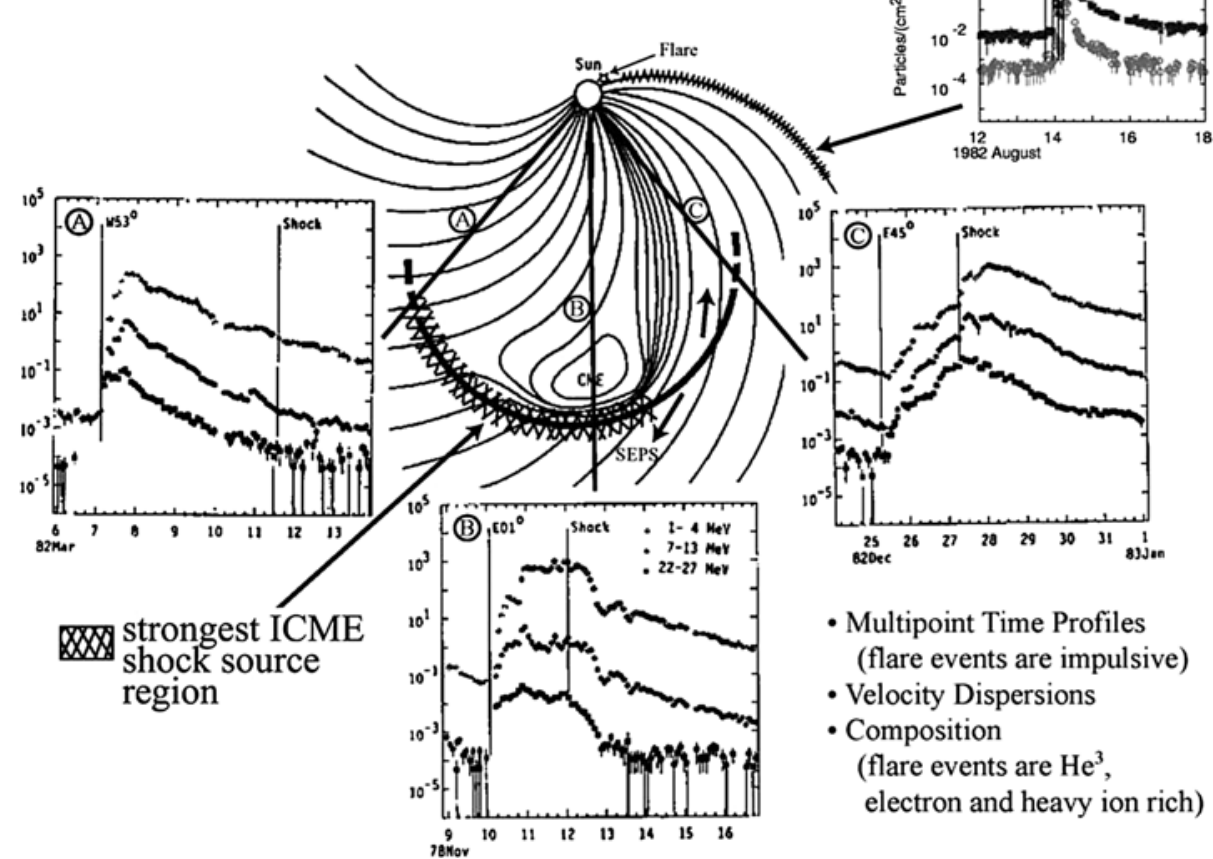

Fig. 6 A classic picture adapted from Cane et al. (1988) showing the spatial organization of time profiles of SEP events with respect to a CME location. STEREO observations will regularly test this picture, and refine it, with multipoint and ion composition observations

profiles of the particles depend on the longitude of the associated solar event as illustrated in Fig. 6. This paradigm-defining result was investigated with earlier multi-spacecraft observations, primarily using data from IMP-8 and the two Helios spacecraft. The interpretation is that particle intensities peak near the nose of the shock and decline on the flanks (Reames et al. 1996). Because of the generally spiral nature of the interplanetary magnetic field, an observer's connection point to a shock swings eastward with time across the face of the outward-moving shock through an angle of $\sim 50-60$ degrees. The observer remotely senses the particle acceleration when it magnetically connects to the shock. With IMPACT on the two STEREO spacecraft, two different points along the shock can routinely be sampled simultaneously, allowing separation of these spatial and temporal effects as the shock travels outward.

The primary tool for understanding the SEP acceleration physics in recent years has been measurements of the abundances of elements and charge states in SEP ions and their variation in space and time. Systematic variations in abundance patterns, for events with different source longitudes were first reported by Breneman and Stone (1985). An illustration of the evolution of the ion composition in one gradual event is shown in Fig. 7. Events at western longitudes peak early and are initially Fe-rich, the ion composition presumably having been affected by flare-like processes in the hot corona (e.g. Cane et al. 2003). In contrast, 
Fig. 7 Illustration of the variation of ion composition through a gradual SEP event detected by both GOES and WIND
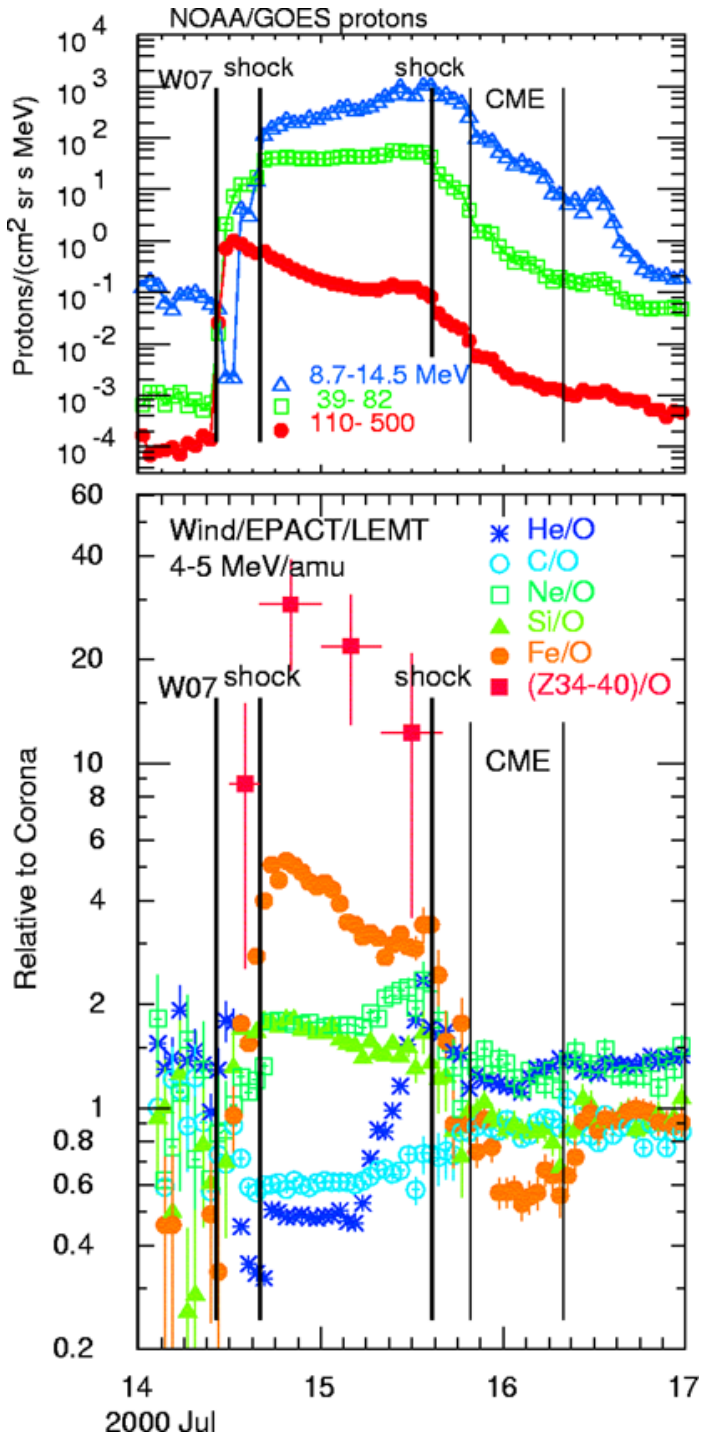

central disk and eastern events are relatively Fe-poor, peak later, and contain larger contributions of particles with solar wind-like composition. Adequate abundance measurements were not available on Helios to allow multipoint ion composition studies. Nevertheless, theoretical analyses suggest that as protons stream away from the shock they generate Alfven waves that trap the particles that follow, causing efficient acceleration to higher energies (Lee 1983, 2005). The spectrum of these self-generated waves is related to the proton energy spectrum and varies with space and time, throttling the flow of SEP ions away from the shock. Ions of the same velocity, but with different charge-to-mass ratios, $Q / M$, resonate with waves of different wave number, $k$. Thus, the relative abundances of different ions that leak away from the shock probe the wave spectrum in the vicinity of the shock. Recent measurements have made it possible to analyze the time dependence of the ion abundance 
variations (Tylka et al. 1999; Reames and Ng 1998), and to develop time-dependent theoretical models of the acceleration and transport of SEPs ( $\mathrm{Ng}$ et al. 1999) that explain these variations. The initial abundance variations depend strongly on the (unseen) spectrum and intensity of high-energy protons back at the shock when it is still near the Sun. With IMPACT instruments, the quantitative relationship between shock parameters, proton spectra, and abundance variations can be sorted out. It may also be possible to use SEP abundance observations to remotely sense the properties of an oncoming shock and forecast its subsequent behavior. STEREO IMPACT, with its SEP composition measurements, provides the opportunity to study abundances on spatially separated spacecraft, allowing us to infer the spatial distribution of the wave spectrum along the surface of a remote shock and its evolution with time. Large spacecraft separations (20-180 degrees) are best for such analyses given the extensive spatial structure of the gradual SEP events.

The leakage of particles from the shock acceleration region produces a spectral "knee" as acceleration becomes less efficient. In some events the energy of this knee can be at $15 \mathrm{MeV}$, in others it is over $100 \mathrm{MeV}$. Understanding the relationship between the knee energy and the properties of the CME-driven shock is essential for determining how rare, extreme SEP events are produced. IMPACT data are expected to reveal new aspects of the physics of particle acceleration that can help us better understand the origin of the SEP spectral knees, at least up to the $\sim 100 \mathrm{MeV}$ energy limit of IMPACT HET measurements. In general, the ability to measure the energy spectra arising from different locations on the same shock on two spacecraft is ideal for testing previously held ideas about the origins of different spectral features.

Although most major SEP ion events are a result of CME-driven shock acceleration, the electron-rich impulsive SEPs arise from flares and yield unique information about the flare process. Flare-heated electrons above $20 \mathrm{keV}$ produce hard X-ray bursts when they interact with the solar atmosphere. Other accelerated electrons stream outward on open coronal field lines to produce type III radio bursts. Current theory suggests that electron beams produce waves between the gyrofrequencies of $\mathrm{H}$ and ${ }^{4} \mathrm{He}$ that are resonantly absorbed by the rare isotope ${ }^{3} \mathrm{He}$ so as to enhance the ${ }^{3} \mathrm{He} /{ }^{4} \mathrm{He}$ ratio by factors of $\sim 1,000$ in the impulsive events (e.g., Roth and Temerin 1997). Ten-fold enhancements are also produced in the abundance of heavy elements such as Fe. The IMPACT investigation will enable coordinated SEP composition and SWAVES radio burst measurements, allowing in-depth analysis of flare acceleration processes that can be contrasted with the processes which accelerate particles at ICME-driven shocks. Recent work using energetic electron events and Type III radio bursts from WIND and ACE show that there is often a large temporal offset between a Type III burst, and the associated electron event (Krucker et al. 1999; Haggerty and Roelof 2002). The origin of this offset is still being debated. With IMPACT and SWAVES investigations on the two spatially-separated STEREO spacecraft, SEP electrons and Type III radio bursts can be compared at two points. Mapping the onset times back to the Sun, we can remotely probe the source(s) at the injection site(s).

Energetic particles streaming out from the Sun can also be used to map the topology of magnetic field lines in the solar wind (Kahler and Reames 1991; Bothmer and Schwenn 1996; Larson et al. 1997) The high and low energy suprathermal electron observations often have different anisotropy signatures (Lin and Kahler 1992; Kahler and Reames 1991) and thus provide complementary information on magnetic field topology. Large decreases in energetic ion intensity are often observed during passage of ICMEs (e.g., Mazur et al. 1998; Cane 2000), probably indicating the inability of the shock-accelerated particles to penetrate significantly into the closed field regions of the ICMEs. The separation of the two STEREO 
spacecraft allows us to determine if the topological and source properties inferred from the particle anisotropies and ion composition are consistent with current paradigms (e.g. as summarized in Reames 1999; Kahler 2001).

It has been argued, based on available observations, (e.g. Kahler 2001; Desai et al. 2006) that the presence of a significant prior background of suprathermal ions from previous CMEs or flares generally increases the intensity of gradual SEP events. While this idea is still under debate, it is intuitively clear that preacceleration should enhance any subsequent acceleration by a statistical process at the shock. Several of IMPACT's detectors, especially SIT, are designed to address the seed population problem observationally. In particular the multipoint sampling on STEREO will reveal how uniform or nonuniform such populations can be, and whether the shock portions with seed populations are more SEP-productive. Observations often show particle spectra of the same intensity and shape over large spatial regions (Reames et al. 1997), from which it has been inferred that particles may be effectively trapped behind shocks. There is some evidence that these trapped particles can form a seed population for SEP generation by a second fast CME near the sun. Later SEP events in a series of SEP events often have higher intensities than the first, suggesting that extreme events may be more easily generated when multiple CMEs occur (e.g. Gopalswamy et al. 2003). An understanding of seed populations in general is a high priority for IMPACT measurement analyses.

Like CME simulations, SEP event models are coming of age as a natural extension of the progress in developing sophisticated coronal and heliospheric numerical models. Several efforts described in the literature (e.g. Lario et al. 1998; Li et al. 2003) make use of MHD model simulations of interplanetary shocks as the basis for SEP spatial distribution and time profile descriptions that can be compared to multipoint or single spacecraft observations. The existence of the IMPACT modeling efforts and others are expected to lead to realistic and detailed SEP event descriptions that are consistent with both the SEP observations on STEREO and the related CME and ICME measurements and models.

\subsection{The Solar Origins of Geoeffectiveness}

Magnetic indices based on ground magnetic perturbations, levels of ionospheric disturbance, relativistic electron and other radiation belt enhancements, and auroral activity are just a few of the commonly used measures of the geoeffectiveness of solar wind events. The parameters from solar wind monitors most widely associated with disturbed conditions include one or more of high bulk speed, high ion density (or dynamic pressure), and strong magnetic field, particularly when the field contains a southward component in GSM coordinates (e.g. see Luhmann 1997; Webb et al. 2000, and references therein). While solar wind stream interaction regions contain enhanced values of the geoeffective parameters, the largest and longest-lived enhancements are from ICMEs (Lindsay et al. 1995) or combinations of stream interaction regions and ICMEs (Tsurutani et al. 1992). In particular, fast ICMEs preceded by interplanetary shocks produce the major geomagnetic storms (Gosling et al. 1990, 1991; Gosling 1993) because of the high dynamic pressures and strong magnetic fields, often with strong southward components, commonly associated with such disturbances, all of which are made more geoeffective by accompanying high bulk speeds. Thus the primary questions raised above about the physics behind the CME speed, and its solar wind stream structure context, are highly relevant to the geoeffectiveness issue.

The basic physics of geoeffectiveness is well known from the viewpoint of solar windmagnetosphere interaction physics (e.g., Kivelson and Russell 1995), but it is not clear how 
geoeffective structures at $1 \mathrm{AU}$ relate to the transients seen at the Sun. In particular, the coronal conditions leading to eruptions with high speed ejecta carrying intense southward magnetic fields, the recipe for geoeffective ICMEs, is not understood. For example, sometimes an apparent fast halo event in LASCO images, thought to portend an important Earth-bound ICME, is not followed by a significant solar wind event at 1 AU. An ICME may change speed and character as it propagates to Earth, rendering it less locally impressive than inferred from coronagraph images, or it may miss the Earth altogether. Similarly, an event may lead to an impressive L1 disturbance in which the bulk of the large out-of-ecliptic fields are northward. Geoeffectiveness thus depends on the heliolongitude dependence as well as the intrinsic nature of ICME properties and those of the ambient wind into which it propagates. IMPACT measurements combined with L1 measurements will provide 2- and 3-point information on the geoeffective extent of specific events observed by the imagers on STEREO. For example, proxy calculations of geomagnetic indices such as Dst (Lindsay et al. 1999b) and Kp (Detman and Joselyn 1999) based on solar wind parameters for each spacecraft can give a measure of the potential geoeffectiveness of a particular event along a significant segment of Earth's orbit. IMPACT team modeling of specific events can help to establish the observational and physical basis for relating geoeffectiveness to solar observations.

Monitors at L1 and other upstream locations do not always give a good indication of the solar wind control of the magnetosphere because they are generally not on the stagnation streamline that reaches the magnetopause. Multispacecraft studies upstream of the bow shock suggest that the coherence length of solar wind structures is sometimes smaller than the separation of the monitors (Paularena et al. 1999), and studies of structure orientations show that they often do not move in planar fronts perpendicular to the Earth-Sun line. Structures strongly influenced by the corotating solar wind streams tend to align themselves along the Parker Spiral direction (Richardson and Paularena 1998), while high speed transients with large internal pressures often give the impression that the incident structure is perpendicular to the Sun-Earth line (e.g. Lindsay et al. 1994). Numerical simulations of the magnetosphere-solar wind interaction show that the angle of incidence of interplanetary structures affects the magnetosphere's response, while simulations of ICME propagation in a structured solar wind show how complicated they can become in the course of their interplanetary evolution (as discussed above). IMPACT observations in the STEREO configuration with an ever-widening baseline between spacecraft provide a controlled experiment for measurements of coherence scale lengths of solar wind structures, including ICMEs and solar wind stream interaction regions. They also form the basis for determining the normals and curvatures of shocks and the changes in the appearance of ICMEs over the varying baseline, allowing reassessment of needs for positioning solar wind monitors and future interplanetary constellation spacecraft. IMPACT 3D heliospheric models consistent with the observed solar wind and ICMEs, used to drive 3D MHD magnetosphere models, are expected to provide insights into the Earth's response not possible with L1 observations alone.

\subsection{The Solar Magnetic Cycle}

The photospheric magnetic field is dominated by the cycle of emergence and decay of active regions that somehow participate in the periodic reversals of the global solar field. During the course of this dynamo-driven cycle, the amount of flux threading the photosphere changes by a factor of $\sim 5$ in the present epoch (e.g., Schrijver and Harvey 1994). Eclipse and coronagraph observations and coronal models based on photospheric field observations show that the corona responds to changes in the photospheric field, but it is not always 
obvious how such changes affect the heliospheric magnetic field (McComas et al. 1992; McComas 1995). Some studies suggest a factor of two change in the total interplanetary magnetic flux over the cycle (e.g. Luhmann et al. 1998). Both the loop-like appearance of CMEs and the measurements of counterstreaming electrons in ICMEs indicate that new solar magnetic flux opens into interplanetary space during transients, but on the average, newly opened solar flux must be counterbalanced by newly closed flux as the solar magnetic field does not become increasingly open over time. Yet direct interplanetary signatures of magnetic flux closing down at the Sun, which would produce heliospheric field lines disconnected from the Sun, have been elusive. If magnetic flux tubes are completely disconnected from the Sun, electron heat flux dropouts would be expected and are observed during disconnection events that occur well away from the Sun at the heliospheric current sheet (Gosling et al. 2005b), but these do not appear to be present in equal numbers with counterstreaming electron intervals (indicators of closed field lines), or are masked by some other process. Those dropouts that have been detected are concentrated in the vicinity of the heliospheric current sheet in the slow solar wind (McComas et al. 1989).

STEREO IMPACT will provide sensitive measurements of suprathermal electron angular distributions over broad angle and energy ranges, together with interplanetary magnetic field measurements, at two low-heliolatitude sites. These measurements will not, in general, be contaminated by suprathermal electrons originating from Earth's bow shock, as is often the case at L1. Thus IMPACT provides the best opportunity to date to utilize suprathermal electron observations in determining the overall evolving topology of the heliospheric magnetic field and to study the opening and closing of magnetic flux in the solar environment. Coordination with the SWAVES experiment allows assessment of the effects of scattering on suprathermal electron distribution at locations where the electron heat flux drops outs. The dual-site measurements and imaging that STEREO affords make it possible to compare suprathermal electron distributions and anisotropies at different spacecraft separations and to compare those distributions with prevailing conditions at the Sun and in the corona.

The solar dynamo transforms poloidal field to toroidal field and also results in polar field reversals. Eruption of the active region fields must eventually lead to a loss or relaxation of the toroidal fields, with CMEs playing a part in this process (e.g., Rust 1994). Above it was pointed out that the magnetic structures of those ICMEs that can be described as flux ropes are related to the global solar magnetic polarity, with their leading edge fields sometimes corresponding to the orientation of the prevailing helmet streamer belt field. However, the handedness or twists of these structures is not clearly related to the magnetic cycle. In contrast, the magnetic fields observed around active region and polar crown filaments, whose eruptions often accompany CMEs, appear to have a definite organization of handedness. Right-handed fields seem to prevail in the southern hemisphere, while left-handed fields prevail in the north (Bothmer and Schwenn 1994). The relationship between the magnetic field of a filament and its surroundings, and the magnetic field that is observed in an ICME, remains an open question (e.g. Leamon et al. 2004). Some regard the filament and its local overlying fields as the source of the ICME flux ropes, while others consider the former is a small part of a much larger erupting coronal structure that supplies most of the interplanetary flux rope via coronal reconnection. IMPACT field and electron measurements, coupled with PLASTIC measurements of the plasma ion composition, can be used to distinguish the magnetic fields in the filamentary material from those of other parts of the ICME structure, and examine their relationship, an important step toward resolving the source region question (e.g. Gloeckler et al. 1999).

Additional insights into the solar magnetic cycle come from comparing the characteristics of both ICMEs and solar wind structure for different cycles given the behavior of the 
photospheric field (e.g. Wang and Sheeley 1990a; Luhmann et al. 2002; Li and Luhmann 2006). Fairly continuous interplanetary data sets from L1 exist from ISEE-3 (1978-1987), WIND (1996-present) and ACE (1998-present) missions. Although the STEREO mission will have a lifetime limited by resources and orbital evolution, it will observe the rise to the cycle 24 maximum for comparison with the rise to cycle 21 maximum observed by ISEE- 3 and the rise to cycle 23 maximum observed by WIND and ACE. The observed properties of the active regions can be compared with the number and nature of the ICMEs detected by IMPACT. While this study could also be carried out with an L1 monitor, the availability of the STEREO multipoint view provides the basis for a clearer interpretation in terms of quantities such as the size and flux content of ICMEs that can then be used in retrospective studies of the earlier data. IMPACT will extend the records of information on features such as ICME flux rope occurrence, polarity, orientation and handedness, providing an improved basis for comparisons with solar dynamo models and their coronal counterparts.

\subsection{Complementary Science Objectives}

\subsubsection{Space Plasma Microphysics}

After decades of effort, uncertainty remains regarding the physics of thermalization of solar wind electrons at collisionless shocks. As the electrons encounter the shock, they are thought to move adiabatically through the layer under the influence of the cross-shock potential, leaving an inaccessible region of phase space downstream (e.g., Feldman et al. 1982; Goodrich and Scudder 1984). It has been suggested that this form of the downstream distribution function is unstable to the two-stream instability (Gedalin 1999). Furthermore, intense nonlinear electrostatic waves and phase space holes have been observed near the overshoot of collisionless shocks (Bale et al. 1998a), a possible signature of nonlinear evolution in a two-stream instability. As a shock passes over each STEREO spacecraft, IMPACT and SWAVES operating together in the burst mode can capture detailed distribution functions and plasma wave waveforms, allowing us to systematically study the evolution of both the waves and electrons in the shock layer.

The radio emission observed during an IP Type II or III radio burst is generated at $1 \mathrm{f}_{\mathrm{pe}}$ and/or $2 \mathrm{f}_{\mathrm{pe}}$ by a process known as plasma emission. The microphysics of the plasma emission process underwent a paradigm shift due to WIND observations of elliptically polarized plasma waves at $1 \mathrm{f}_{\mathrm{pe}}$ in the source of these bursts, where longitudinally polarized Langmuir waves were expected (Bale et al. 1998b; Kellogg et al. 1999). This observation implies strong scattering or possibly wave trapping. The SWAVES experiment on STEREO measures all 3 electric field components of these waves, while IMPACT provides essential detailed information on the electron beams driving this process. Other related observations indicate that the Type II source can be probed in situ (Bale et al. 1999). The radio emission comes from near quasi-perpendicular connections to the ICME-driven shock, while the shock-accelerated electrons show the shock surface is highly structured, as mentioned earlier, giving rise to multiple emission sites. Together, SWAVES and IMPACT can further resolve the Type II source nature, allowing improved inference of the shock structure from remote energetic particle and radio measurements.

\subsubsection{CIRs and SIRS}

From WIND experience, we know that CIRs (Corotating Interaction Regions) (and sometimes non-repeating SIRs, Stream Interaction Regions) are the source of much of the lower 
energy interplanetary energetic particle population during periods of low solar activity

(Reames 1999; Desai et al. 2006). While they are not ICMEs, CIRs and SIRs give us valuable information about shock acceleration processes. CIRs and SIRs are formed when highspeed solar-wind streams overtake low-speed solar wind emitted earlier in a solar rotation. A pair of shock waves forms around these regions, the forward shock propagating outward into the slow wind and a reverse shock propagating backward into the high-speed stream (e.g., Gosling and Pizzo 1999). The shocks generally form beyond 1 AU, but a good fraction of L1 stream interaction regions exhibit them as well. The strongest particle acceleration in CIRs and SIRs occurs at the reverse shock. Observations on the WIND spacecraft show distinctive features in these energetic particles, with $\mathrm{C} / \mathrm{O}$ abundances that depend upon stream speed (e.g., Mason et al. 1997); evidence of cross-field particle transport in intense events (Dwyer et al. 1997), and spatial distributions of 1-10 MeV/amu particles extending over $226^{\circ}$ in solar longitude (Reames et al. 1997). The CIR and SIR particles may also be an important source of suprathermal seed populations for subsequent ICME shock acceleration. STEREO IMPACT provides a unique opportunity for multi-point composition measurements of the CIR and SIR-associated ions as these enormous 3D structures rotate past the spacecraft.

\subsubsection{ACRs}

ACRs (Anomalous Cosmic Rays) are believed to be produced when interstellar pickup ions are carried out by the solar wind and accelerated at the heliospheric termination shock (Fisk et al. 1974). Subsequently they make their way back into the inner heliosphere against the flow of the solar wind. Because pickup ions are selectively accelerated by the termination shock primarily due to their high injection speeds, ACRs provide information on the role of the injection process in determining the maximum particle intensities of SEPs from CME/ICME-driven shocks. IMPACT provides sensitive coverage of the low-energy (1-30 MeV/nucleon) ACR ions at a time in the solar cycle when their spectrum is least modified by transient interplanetary structures, giving a truer picture of their source spectrum at two heliospheric locations.

\subsubsection{Reconnection Physics}

It has recently been demonstrated (Gosling et al. 2005a, 2005b, 2006; Phan et al. 2006) that local, quasi-stationary reconnection occurs relatively frequently in the solar wind and produces pairs of Petschek-type exhausts i.e., exhausts bounded by Alfven or slow mode waves emanating from reconnection sites. These exhausts occur as brief (typically minutes) intervals of roughly Alfvenic accelerated or decelerated plasma flow confined to magnetic field reversal regions that usually take the form of bifurcated current sheets. The overall transitions from outside to inside the exhausts typically, but not always, are slow-mode-like on both sides. Multi-spacecraft observations by ACE, Wind, and Cluster at 1 AU demonstrate that the exhausts result from local quasi-stationary reconnection at extended $\mathrm{X}$-lines, in one case persisting for at least 2.5 hours along an X-line that extended for at least $2.5 \times 10^{6} \mathrm{~km}$ (Phan et al. 2006). These are lower limit estimates based on the limited spatial separations available for the spacecraft. In order to extend these estimates to larger scale sizes and longer times, larger spacecraft separations are required. Observations by IMPACT and PLASTIC on the dual STEREO spacecraft will allow us to determine the overall spatial and temporal scale of reconnection in the solar wind. Such determinations will have important implications for models of the reconnection process in other environments such as the solar atmosphere and Earth's magnetosphere. 


\subsubsection{Space Weather Applications}

In support of STEREO's space weather beacon activity (see Thompson et al., and Biesecker et al., this volume, 2007), IMPACT supplies 1 min averaged basic solar wind plasma and suprathermal electron, magnetic field, and SEP ion and electron information processed onboard (in the IDPU) to conform to the designated telemetry allocation. The IMPACT beacon data contents are summarized in Table 2. Synchronized PLASTIC plasma ion beacon data are also produced in the IMPACT IDPU. IMPACT team members experienced in supplying data to the ACE RTSW system (see Garrard et al. 1998), an effective prototype for the STEREO beacon system, has led to an active IMPACT role in the organization of the in situ beacon data at the STEREO Science Center. IMPACT also includes team members from the NOAA Space Environment Center (SEC) who act as interfaces between our investigation and their operations, enabling forecaster use of STEREO IMPACT beacon data (also see Biesecker et al., this volume, 2007).

The plasma and magnetic field information from the trailing spacecraft can be used by forecasters to anticipate the arrival at Earth of corotating structures. In particular, if a high speed solar wind stream is detected, a forecast can be made of an imminent magnetospheric relative electron increase and a modest magnetic storm from the associated stream interaction region. The SEP beacon data also allow forecasts of the arrival of an interplanetary shock and its ICME driver. If both leading and trailing spacecraft detect a gradual SEP event, the probability of the shock and ICME impacting Earth's magnetosphere squarely is high. Moreover, if the SEP event observed at the leading spacecraft has a rapid rise-time, it is likely that the shock nose is between the two STEREO spacecraft-a situation favoring a local gradual SEP event including an ESP component on shock arrival at Earth, plus geomagnetic storm consequences of the ICME. Any SEP information from upstream of Earth could be combined with this information to further diagnose the shock strength and nose position with respect to the Sun-Earth line.

A forward-looking issue for NASA is concern over radiation safety during interplanetary human spaceflight, in particular to the Moon and Mars. Reports dealing with the potential hazards to astronauts (e.g., Zeitlin et al. 2004) point out the need to monitor solar activity from many viewpoints to ensure adequate warnings of potential large SEP fluxes. LRO (Lunar Reconnaissance Orbiter), the first vision for Space Exploration mission launching in 2008, carries an energetic particle telescope that will measure the SEP events STEREO IMPACT will detect from other perspectives. Together, these measurements will provide a demonstration of the sensitivity of the local SEP environment to heliospheric location. IMPACT also provides an occasional upstream solar wind monitor for Mars missions. Mars Express includes some in situ particle instrumentation, making coordinated studies of Mars space weather with STEREO possible.

\subsubsection{Relationships to Other Missions and Ground-Based Observations}

STEREO represents part of NASA's evolutionary sequence of solar/interplanetary missions in the SEC Roadmap that targets the 3D Sun and inner heliosphere, and space weather's underlying causes. STEREO IMPACT investigation measurements build on the information gained primarily from IMP-8, Helios 1/2, ISEE-3, PVO, Ulysses, WIND, and ACE, the latter three of which are still operating. IMP-8 observations led to the first work on the magnetic flux rope models of ICMEs (Lepping et al. 1990 and references therein), ISEE-3 provided long-term detailed in situ measurements at L1 from which solar cycle variations of ICME occurrence and internal information on ICMEs including electron anisotropies were 
derived (Gosling et al. 1987). Helios $1 / 2$ enabled the first in situ look at solar wind character and transients into 0.3 AU (Bothmer and Schwenn 1998). PVO provided an effective $0.7 \mathrm{AU}$ outpost to measure solar wind behavior at Venus, allowing both evaluation solar wind monitor for space weather purposes closer to the Sun than L1 (Lindsay et al. 1999a), and study of CME/ICME relationships in a quadrature configuration with SMM and Solwind coronagraphs at Earth (Lindsay et al. 1999b). Ulysses explored the high latitude heliosphere, first during low solar activity conditions (e.g., Phillips et al. 1995) and then during high solar activity conditions (e.g., McComas et al. 2003), revolutionizing our in situ perspective of the 3D heliosphere. Ulysses in situ data and SOHO images were also used in efforts to match solar signatures of CMEs with ICMEs (Funsten et al. 1999). However, combined ACE, WIND and SOHO studies (e.g. Webb et al. 2000) provide what are perhaps the best examples of what can be accomplished with the planned STEREO imaging, SWAVES, PLASTIC, and IMPACT measurements in combination. The GOES spacecraft is of course another reliable source of near-Earth information on SEP protons and solar X-rays with a long history, though its SEP measurements are limited by its magnetospheric location.

On the complementary imaging side, SOHO, TRACE and RHESSI continue to provide solar EUV and X-ray imaging of the corona for use in interpretive studies (e.g. Klein et al. 2005). Earthward-directed CMEs are observed as halo events on SOHO. SMEI on Coriolis (Jackson et al. 2004) provides wide-field coronagraph images from an Earth-orbiting Air Force satellite platform, and is ideally suited for STEREO HI comparisons and in situ comparisons. SXI on NOAA's GOES-M approximates Yohkoh soft X-ray images at a 1-minute cadence in a softer, more sensitive, X-ray band geared toward coronal studies (Pizzo et al. 2005). Both these imagers complement STEREO imaging, and enhance IMPACT science by providing a more complete description of the coronal events preceding detected ICMEs. Solar-B, to be launched around the same time as STEREO, adds a unique coordination capability in that it allows detailed looks at the active regions giving rise to the CME events STEREO will observe. For example, the vector magnetic field measurements obtained with Solar B can be used to assess the nonpotentiality, polarity, and twist of the magnetic fields in the active regions giving rise to detected ICME structures. Planned campaigns are required (e.g. see Thompson et al., this volume, 2007) given Solar-B's smaller high resolution imaging field of view. However, Solar-B also has a full-disk Yohkoh-like soft X-ray imaging capability that can enhance STEREO data interpretation more generally. Solar Dynamics Observatory (SDO) may launch before the STEREO mission is over. SDO would provide full disk vector magnetograms that could add significantly to the solar connections analyses and IMPACT interpretive modeling. The SDO images are also expected to be returned at a higher rate than those from SOHO, showing more details of time-dependent solar and coronal features.

Opportunities also exist for organizing opportunistic inner heliosphere constellations by combining interplanetary plasma, magnetic field, and SEP observations from several planetary missions. MESSENGER, destined to reach Mercury in 2015 obtains limited in situ particle and field measurements during its cruise phase. However, in Mercury orbit MESSENGER provides an inner heliosphere outpost for space weather measurements. Venus Express and Mars Express respectively provide in situ measurement outposts at Venus and Mars, as mentioned earlier. There is already a plan in place at CESR to combine the data sets from the SWEA-like instruments on Mars Express and Venus Express with STEREO IMPACT SWEA measurements on a regular basis. The possibilities for exploiting the heliospherewide models of observed STEREO events to interpret these widely distributed measurements are potentially ground-breaking in terms of future Heliospheric Sentinels mission 
planning. The planetary missions also benefit from STEREO's physically separated measurements given their own needs to interpret solar wind interaction effects.

The importance of ground-based instruments to STEREO, especially those providing information on solar magnetic fields, cannot be overstated. Two particular ground-based resources that deserve highlighting because of their importance to STEREO-related modeling are the SOLIS full-disk vector magnetograph installations, and the GONG near-24 hr solar field monitoring network. Together with MDI on SOHO, these observatories provide the synoptic maps and local magnetograms that make possible unifying coronal, heliospheric and SEP event modeling (see Aschwanden et al., this volume, 2006). Photospheric field maps are already used at NOAA SEC to make predictions of the solar wind speed and interplanetary field polarity based on the Wang/Sheeley/Arge semi-empirical approach (Arge and Pizzo 2000; at http://sec.noaa.gov/wsa/), at the LMSAL Solar Soft site for coronal holes and solar wind source mapping (http://www.lmsal.com/solarsoft/latest_events/, also see Thompson et al., this volume, 2007), and at CCMC as boundary conditions for 3D MHD models of the corona and solar wind (http://ccmc.gsfc.nasa.gov). The SOLIS full-disk vector magnetograms and maps are expected to revolutionize the capabilities of models to describe CME initiation and propagation more accurately. GONG magnetograph data currently provide the best possibilities for continuous and high cadence magnetogram coverage from the ground. KPNO, MWO and WSO magnetograms, MLSO coronagraph images, H-alpha and other measurements from NSO, ISOON and other active solar optical and radio observatories linked to some of the above websites contribute further information toward interpreting what is deduced from the combined STEREO imaging and in situ observations.

\section{IMPACT Instruments Overview}

\subsection{Overview and Spacecraft Accommodation}

The IMPACT investigation involves engineers, technicians and scientists in 19 institutions. Table 1a provides a list of the team members and their affiliations. Responsibilities for the instrumentation are summarized in Table 1b. IMPACT includes hardware contributions from both university and NASA centers, as well as from international partners. Its overall development was guided by technical Project Manager D.W. Curtis at UCB-SSL. The nature of the instruments and team organization made it advantageous to carry out the development as the two instrument suites shown in Fig. 8a. IMPACT's Boom suite includes the SWEA, STE and MAG sensors, while the SEP suite includes SIT, SEPT, LET and HET sensors. DPU (data processing unit) functions are handled in both a SEP-suite-specific "SEP central" box, and an all-IMPACT IDPU box that provides the single IMPACT interface to the spacecraft commanding and data system. Caltech team member A.C. Cummings adopted day-to-day responsibility for the SEP suite technical oversight.

STEREO spacecraft are non-spinning, so that the particle sensor providers had to take that into account in their designs and spacecraft accommodation plans. The Boom Suite instruments are mounted on a $\sim 5.8 \mathrm{~m}$ telescoping carbon fiber epoxy boom, shown in a stowed configuration in Fig. 8b. The boom is mounted along one corner of each spacecraft in a position that minimizes interference with the high gain and SWAVES antennas, and is deployed antisunward. The instruments at its end are usually in the spacecraft shadow. Boom suite development work was centered at UCB-SSL with other IMPACT institutions providing the SWEA and MAG sensors. The detectors in the SEP suite are mounted in several boxes on the body of the spacecraft as shown in Fig. 8c, with different locations on the Ahead and Behind spacecraft to maintain the desired fields of view with respect to the 
Table 1a IMPACT team

\begin{tabular}{|c|c|}
\hline Institution & Members \\
\hline UC Berkeley & $\begin{array}{l}\text { J.G. Luhmann, D.W. Curtis, P. Schroeder, R. Lin, D. Larson, S. Bale, } \\
\text { J. McCauley, R. Ullrich, P. Turin, P. Berg, S. McBride, N. Craig, } \\
\text { L. Peticolas, M. Hashii }\end{array}$ \\
\hline CESR & J.-A. Sauvaud, C. Aoustin, J. Dandouras, P. Louarn \\
\hline Caltech & $\begin{array}{l}\text { E.C. Stone, R.A. Mewaldt, A.C. Cummings, W.R. Cook, B. Kecman, } \\
\text { R. Leske, A.J. Davis, C.M.S. Cohen, V. Nguyen, H. Miyasaka }\end{array}$ \\
\hline GSFC & $\begin{array}{l}\text { T.T. von Rosenvinge, M.H. Acuna, L.S. Reichenthal, K. Ogilvie, } \\
\text { A. Szabo, D.V. Reames, C.K. Ng, S. Shuman, J. Hawk, K. Wortman }\end{array}$ \\
\hline JPL & M.E. Wiedenbeck, R.G. Radocinski \\
\hline Univ. of Kiel & R. Mueller-Mellin, H. Kunow, S. Boettcher, B. Heber \\
\hline $\begin{array}{l}\text { Research and Scientific } \\
\text { Support Division, ESA }\end{array}$ & T.R. Sanderson, R.G. Marsden \\
\hline JHUAPL & G.M. Mason \\
\hline Univ. of Maryland & P. Walpole \\
\hline MPS & A. Korth, V. Vasyliunas \\
\hline UCLA & C.T. Russell, A. Lam \\
\hline LASP, Univ. of Colorado & J.T. Gosling \\
\hline SWRI & D.J. McComas \\
\hline LANL & H. Funsten \\
\hline DESPA/Meudon & J.-L. Bougeret \\
\hline Univ. of Michigan & T. Gombosi, D. DeZeeuw \\
\hline SAIC & J.A. Linker, P. Riley, Z. Mikic \\
\hline NOAA-SEC & V. Pizzo, D. Odstrcil \\
\hline Univ. of Goettingen & V. Bothmer \\
\hline KFKI & K. Kecskemety \\
\hline
\end{tabular}

nominal Parker Spiral magnetic field orientation in the ecliptic. Figure 9 summarizes the IMPACT particle detectors' fields of view described in the instrument summaries below.

\subsection{Boom Suite}

\subsubsection{SWEA (Solar Wind Electron Analyzer)}

Solar wind electron measurements are essential for investigating the topology and solar connections of solar wind and ICME magnetic fields. SWEA, the IMPACT Solar Wind Electron Analyzer, was provided by the CESR and is described in detail by Sauvaud et al. (this volume, 2007). Its main purpose is to obtain close to $4 \pi$ steradian measurements of solar wind thermal and suprathermal (halo) electrons with sufficient angular and time resolution to allow use of the highly anisotropic heat flux electrons (energies $\sim 300-800 \mathrm{eV}$ ) to characterize the local magnetic field topology and its solar connections.

To accomplish its goals, SWEA measures the distribution function of the solar wind thermal and suprathermal electrons from $\sim 0$ to $\sim 3000 \mathrm{eV}$ with the instrument shown in Fig. 10. SWEA's top-hat $\left(\sim 90^{\circ}\right.$ symmetric quadraspherical) electrostatic analyzer (ESA), with $360^{\circ}$ acceptance angle in a plane, uses electrostatic deflectors to provide \pm 65 degree coverage in elevation out of the plane. Electrons entering the SWEA ESA at different azimuthal angles are focused onto stacked microchannel plate (MCP) detectors at 16 angular locations. 
Table 1b Summary of IMPACT instruments and their basic measurements

\begin{tabular}{|c|c|c|c|c|c|}
\hline Experiment & Instrument & Measurement & $\begin{array}{l}\text { Energy or mag. } \\
\text { field range }\end{array}$ & Time res. & $\begin{array}{l}\text { Instrument } \\
\text { provider }\end{array}$ \\
\hline \multirow[t]{2}{*}{ SW } & STE & $\begin{array}{l}\text { Electron flux and } \\
\text { anisotropy }\end{array}$ & $2-100 \mathrm{keV}$ & $10 \mathrm{~s}$ & UCB \\
\hline & SWEA & $\begin{array}{l}\text { 3D electron distrib., } \\
\text { core } \& \text { halo density, } \\
\text { temp. \& anisotropy }\end{array}$ & $\sim 0-3 \mathrm{keV}$ & $\begin{array}{l}3 \mathrm{D}=30 \mathrm{~s} \\
2 \mathrm{D}=8 \mathrm{~s} \\
\text { Moment. } 2 \mathrm{~s}\end{array}$ & $\begin{array}{l}\text { CESR } \\
+\mathrm{UCB}\end{array}$ \\
\hline MAG & MAG & Vector field & $\begin{array}{l} \pm 500 \mathrm{nT}, \\
\pm 65536 \mathrm{nT}\end{array}$ & $1 / 8 \mathrm{~s}$ & GSFC \\
\hline \multirow[t]{6}{*}{ SEP } & SIT & $\begin{array}{l}\mathrm{He} \text { to } \mathrm{Fe} \text { ions } \\
{ }^{3} \mathrm{He}\end{array}$ & $\begin{array}{l}0.03-5 \mathrm{MeV} / \text { nuc } \\
0.3-0.8 \mathrm{MeV} / \text { nuc }\end{array}$ & $\begin{array}{l}30 \mathrm{~s} \\
30 \mathrm{~s}\end{array}$ & $\begin{array}{l}\text { U. of Md. } \\
+ \text { MPS } \\
+ \text { GSFC }\end{array}$ \\
\hline & SEPT & $\begin{array}{l}\text { Diff. electron flux } \\
\text { Diff. proton flux } \\
\text { Anistropies of } e, p\end{array}$ & $\begin{array}{l}30-400 \mathrm{keV} \\
60-7000 \mathrm{keV} \\
\text { As above }\end{array}$ & $\begin{array}{l}1 \mathrm{~min} \\
1 \mathrm{~min} \\
1 \mathrm{~min}\end{array}$ & $\begin{array}{l}\text { U. of Kiel } \\
+ \text { ESA }\end{array}$ \\
\hline & LET & $\begin{array}{l}\text { Ion mass numbers } \\
2-28 \& \text { anisotropy } \\
{ }^{3} \mathrm{He} \text { ions flux \& } \\
\text { anistropy }\end{array}$ & $\begin{array}{l}2-40 \mathrm{MeV} / \mathrm{nuc} \\
2-15 \mathrm{MeV} / \mathrm{nuc}\end{array}$ & $1 \mathrm{~min}$ & $\begin{array}{l}\text { Caltech } \\
+ \text { GSFC } \\
+ \text { JPL }\end{array}$ \\
\hline & & $\begin{array}{l}\text { H ions flux \& } \\
\text { anistropy }\end{array}$ & $2-13 \mathrm{MeV}$ & $1 \mathrm{~min}$ & \\
\hline & HET & $\begin{array}{l}\text { Electrons flux } \\
\mathrm{H} \\
\mathrm{He} \\
{ }^{3} \mathrm{He}\end{array}$ & $\begin{array}{l}1-6 \mathrm{MeV} \\
13-100 \mathrm{MeV} \\
13-100 \mathrm{MeV} \\
15-60 \mathrm{MeV} / \text { nuc }\end{array}$ & $\begin{array}{l}1 \mathrm{~min} \\
1 \mathrm{~min} \\
1 \mathrm{~min} \\
1 \mathrm{~min}\end{array}$ & $\begin{array}{l}\text { GSFC } \\
+ \text { Caltech } \\
+ \text { JPL }\end{array}$ \\
\hline & $\begin{array}{l}\text { SEP } \\
\text { Central }\end{array}$ & - & - & - & $\begin{array}{l}\text { Caltech } \\
+ \text { GSFC }\end{array}$ \\
\hline $\begin{array}{l}\text { IMPACT } \\
\text { Common }\end{array}$ & $\begin{array}{l}\text { IDPU } \\
\text { (+Mag Analog) }\end{array}$ & - & - & - & UCB \\
\hline
\end{tabular}

The output provides $\sim 22.5$ degree resolution in azimuth and elevation. This type of detector system has been successfully flown on both WIND and FAST.

SWEA is mounted at the end of the $\sim 5.8$ m IMPACT boom, with the magnetometer sensor mounted $\sim 1 \mathrm{~m}$ inboard. This provides a SWEA open field of view over $>\sim 91 \%$ of the sky; with the spacecraft plus solar panels blocking $\sim 3.5 \%$ of the remaining $9 \%$. The wide SWEA field of view is illustrated in Fig. 9. With this capability, the directionality of the suprathermal electrons can be determined even when the interplanetary magnetic field rotates far out of the ecliptic, as is often observed in ICMEs. SWEA also measures the thermal solar wind plasma, or core, electrons, allowing determination of the core/halo relationships and the calculation of bulk solar wind properties from the electron 3D distribution moments. The nominal time resolution for full 3D distributions, and hence moments, from SWEA is $30 \mathrm{~s}$. 
Fig. 8 a STEREO Behind spacecraft, showing the two IMPACT instrument suites. b The IMPACT Boom Suite, including SWEA, STE and MAG instruments. $\mathbf{c}$ The IMPACT SEP suite on the body of the Ahead and Behind spacecraft, including SEPT, SIT, LET and HET

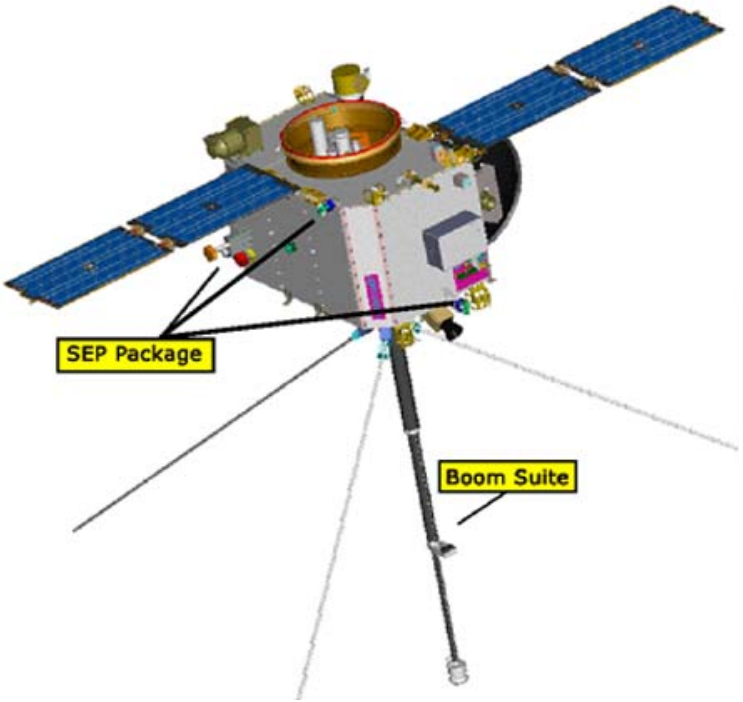

(a)

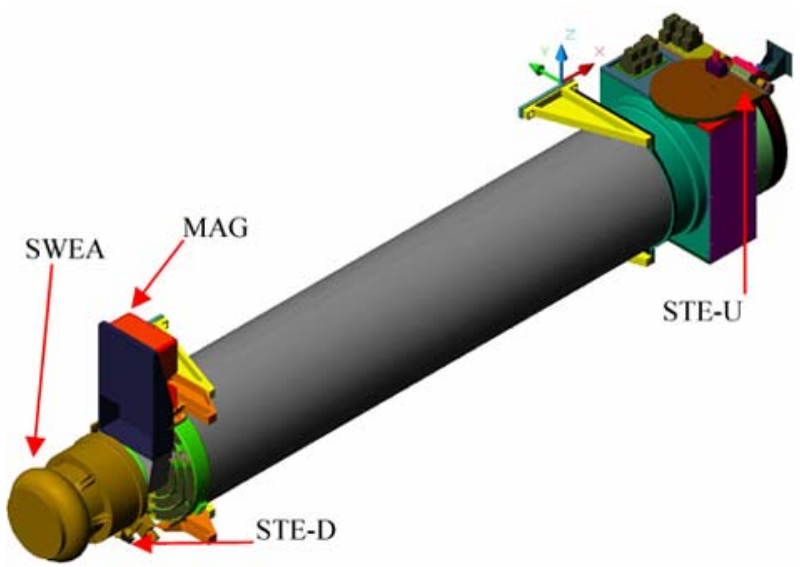

(b)

Measurements of the electron distribution at low energies are affected by the spacecraft potential. An illuminated spacecraft emits photoelectrons and charges up to a few volts positive potential for typical solar wind conditions. The potential of a spacecraft adjusts itself so that the flux of escaping photoelectrons approximately equals the flux of ambient electrons that hit the spacecraft surface. The STEREO spacecraft exterior surfaces are conductive to avoid the related development of large potentials that can strongly distort the incoming electron trajectories. Nevertheless, the low energy portion of the ambient distribution function may not be accurately resolved. IMPACT's design allows the potential of the SWEA instrument to be set to the spacecraft potential so that measurements can be made with full spectral resolution of the analyzer over the entire electron distribution. Such biasing has been successfully implemented on the UCB electron sensor on the Mars Global Surveyor spacecraft. To ensure a minimal impact of spacecraft charging on the SWEA measurements, a NASCAP analysis was performed by Mandell et al. at SAIC (personal communication, 

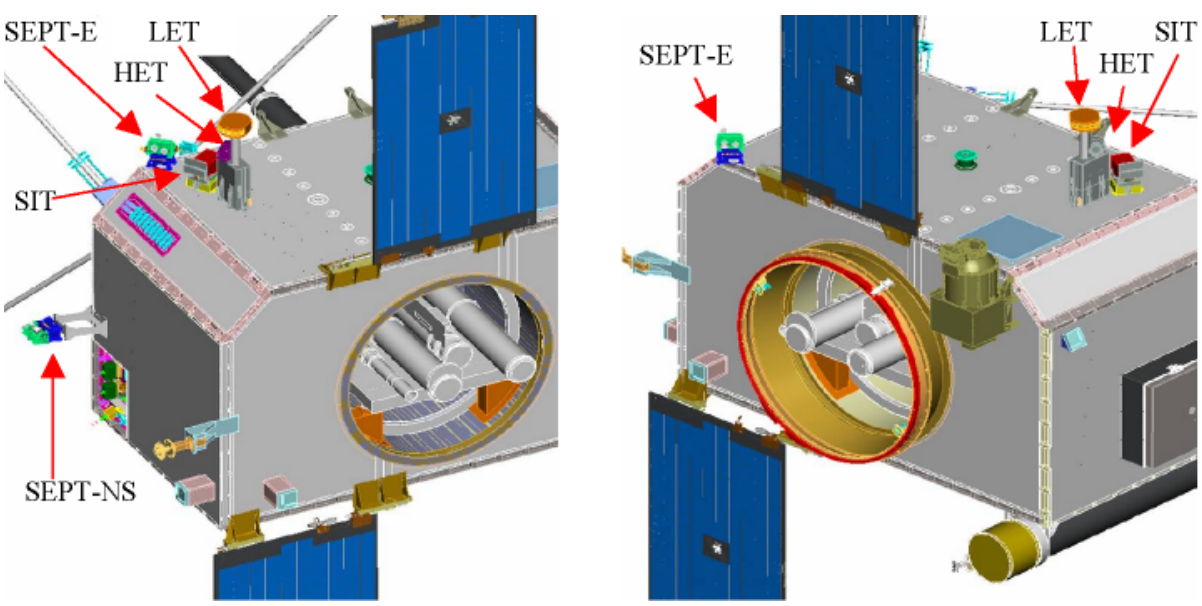

(c)

Fig. 8 (Continued)
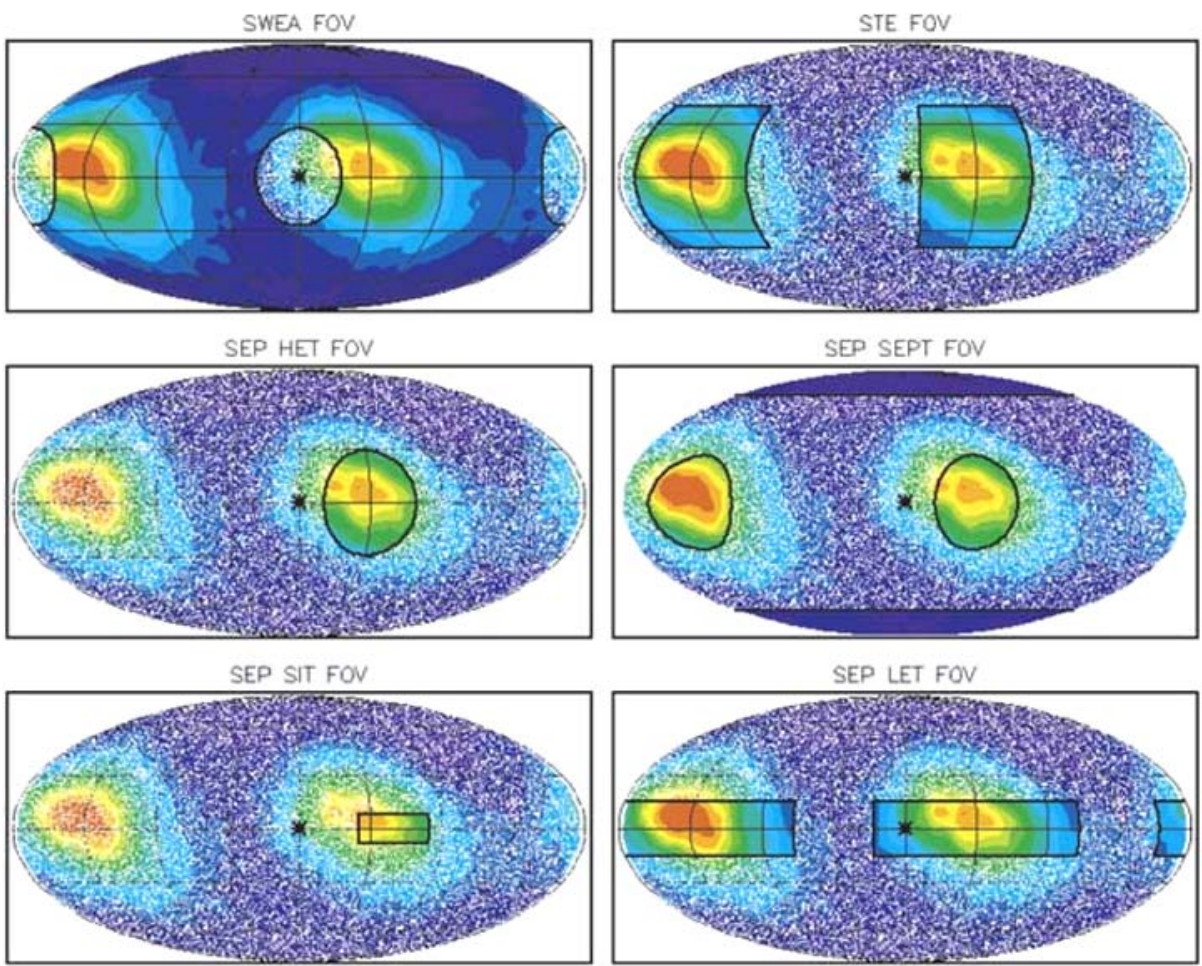

Fig. 9 IMPACT particle sensors fields of view (Reproduced from Luhmann et al. 2005). The color contours show the statistics of the interplanetary field on a mercator projection with the center of the plot toward the Sun. The speckled regions are outside of the field of view. Many of the SEP instruments focus on the nominal Parker Spiral field direction, while the SWEA instrument nearly covers the entire directional sphere 
Fig. 10 Photograph of a SWEA/STE-D flight unit

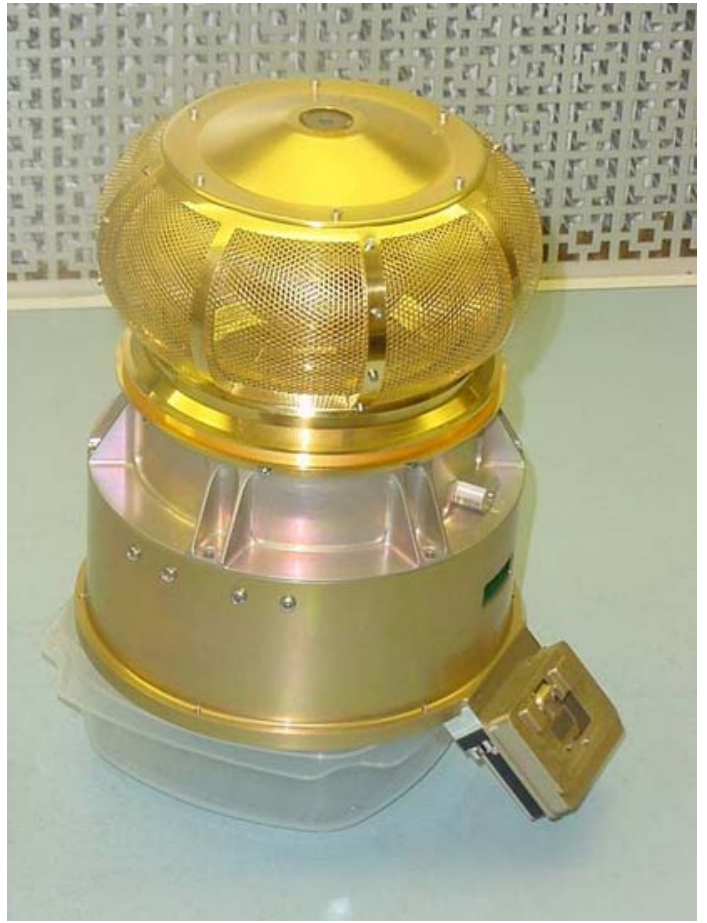

2002). That analysis provided recommendations for materials to use on the spacecraft surfaces. These included black kapton blankets or ITO silver teflon. The notable exception is the solar array front surface (which is non-conductive but always sunlit and so will not charge much). These electrostatic cleanliness measures were regarded as sufficient to preserve the low energy electron measurements given the length of the IMPACT boom.

While the PLASTIC ion analyzer measures the solar wind plasma ion moments that are typically used to characterize solar wind density, bulk velocity, and dynamic pressure, SWEA thermal plasma electron measurements provide a reliable confirmation of the ion moments together with the (anisotropic) electron temperature in different solar wind and CME-related interplanetary structures. Figures 11a,b use analogous WIND 3DP electron data (Larson et al. 1997) to illustrate that accurate electron-based solar wind moments can usually be obtained with a SWEA-like instrument.

Once it is commissioned, SWEA requires only minimum commanding to reset the potential bias mentioned above, if desired. SWEA participates with STE and MAG in an IMPACT burst mode of operation, described below, which allows brief periods of $2 \mathrm{~s}$ full 3D distributions to be obtained for periods of $10 \mathrm{~min}$ around a specified triggering event.

\subsubsection{STE (Suprathermal Electron Telescope)}

STE, the University of California, Berkeley, suprathermal electron telescope, is a new instrument that measures $\sim 2-100 \mathrm{keV}$ electrons moving in the sunward and antisunward directions along the local Parker Spiral interplanetary fields with some angular resolution in the ecliptic plane. Lin et al. (this volume, 2007) describe STE in detail. STE is included in IMPACT to cover solar electrons in the energy range above SWEA that generate type III radio bursts, shock accelerated electrons that produce type II radio emission, and the super- 


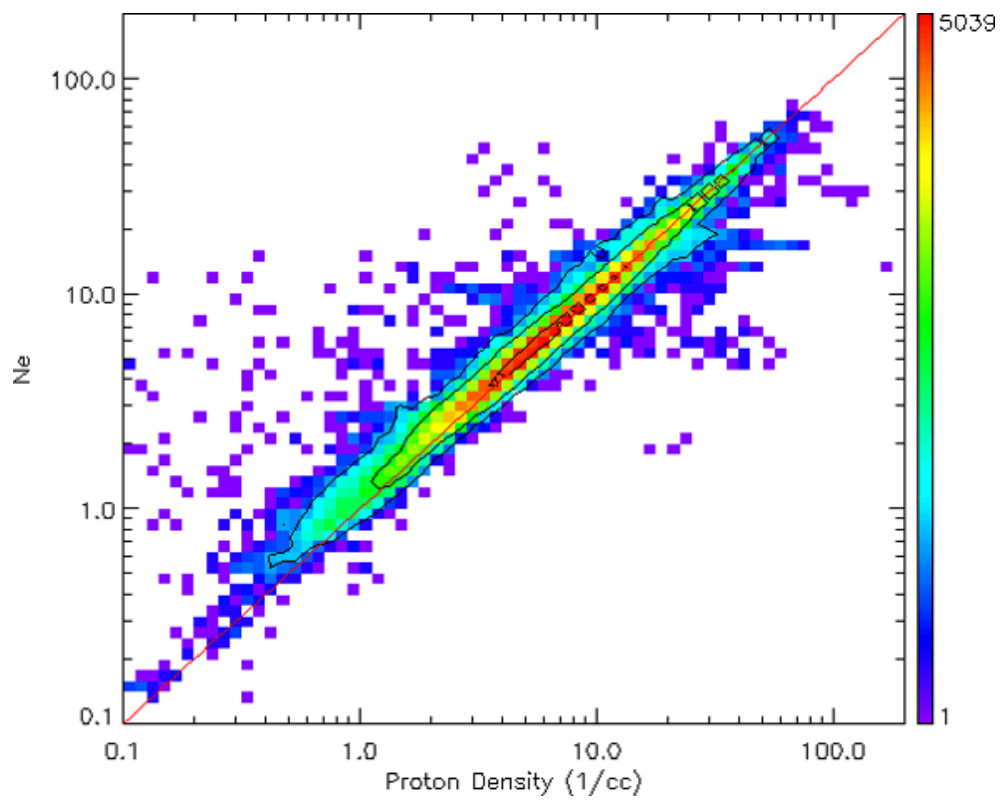

(a)

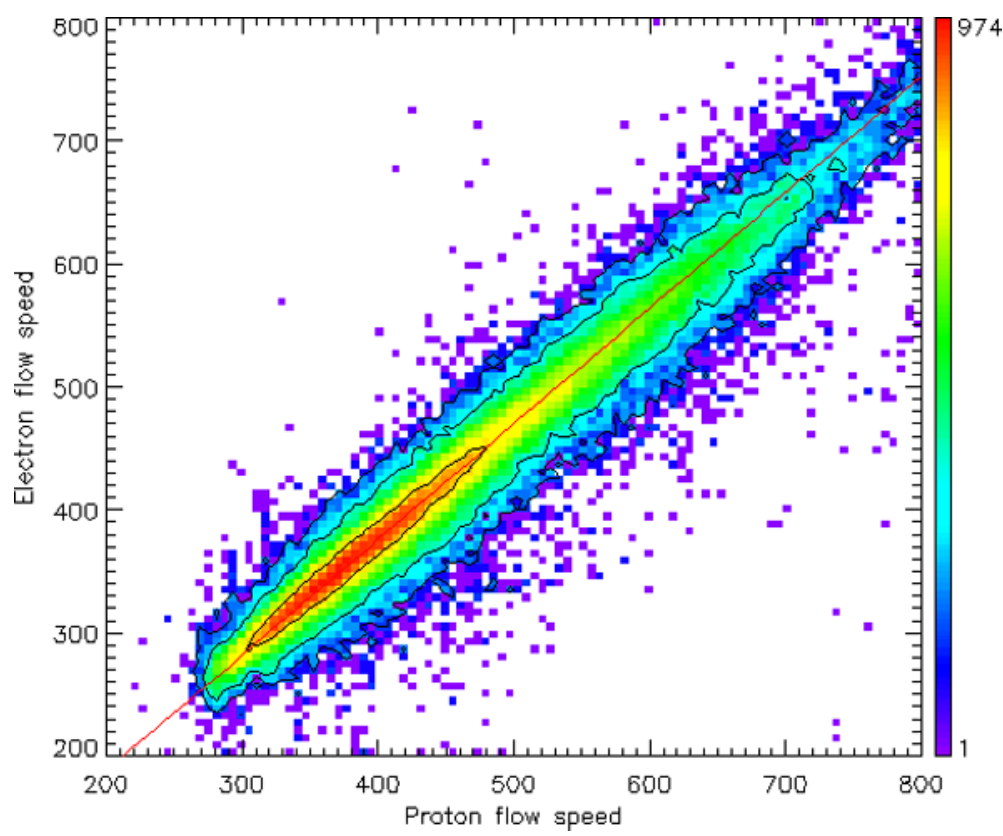

(b)

Fig. 11 a Plasma proton density measured on WIND with 3DP compared with the plasma electron density measurements. 3DP electron measurement techniques are similar to those used for SWEA. b Comparison of plasma bulk speed derived from WIND 3DP proton and electron data. The electrons provide accurate alternate measures of solar wind density and speed under most conditions 
Fig. 12 Photograph of a STE-U flight unit

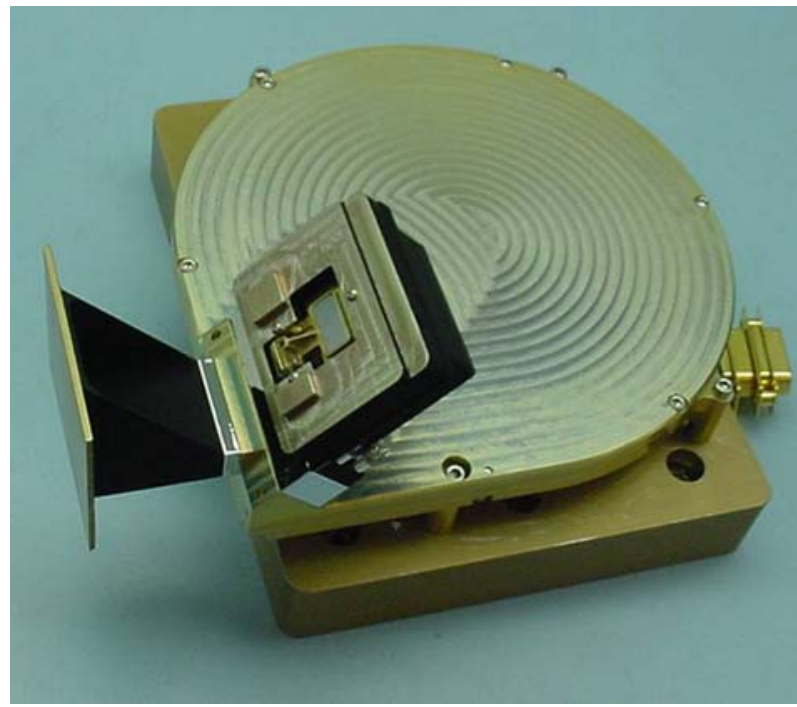

halo electrons (whose origin is unknown) during quiet times. The superhalo was discovered by the high sensitivity electron measurements on WIND using a very large geometric factor electrostatic analyzer, EESA-H. Even so, the count rate above $\sim 2 \mathrm{keV}$ at quiet times was dominated by intrinsic background counts of the very large microchannel plate detector. Thus, weak impulsive electron events could not be detected on wind, and the superhalo observations could not be carried out without long integrations.

The STE sensors provide high sensitivity with low background by using small, low capacitance, low leakage current, passively cooled silicon semiconductor detectors configured in arrays on a wafer, together with low power, low noise electronic chains. Laboratory tests show that electrons down to $\sim 1.5 \mathrm{keV}$ energy can be detected. The overall energy range for stopping electrons is $\sim 1.5$ to $\sim 100 \mathrm{keV}$. STE's intrinsic energy resolution is about $1 \mathrm{keV}$; its telemetered data resolution varies from $\sim 7 \%$ to $\sim 20 \%$ (delta E over E). The STE design uses two arrays consisting of four SSDs in a row. Each array looks through a rectangular opening that provides a $\sim 20 \times \sim 80$ deg. field of view for each SSD $(\sim 1$ steradian solid angle); adjacent fields of view are offset from each other by $\sim 20$ degrees. The customdesigned low power preamp-shaping electronics are mounted next to the SSDs to minimize noise pickup.

A picture of one of the upstream units of STE (STE-U) is shown in Fig. 12. The downstream units (see Fig. 6b) of STE (STE-D) are located at the end of the boom, where they share an electronics box and harness with SWEA. The upstream units of STE (STE-U) are at the boom's sunward end, pointing along the Parker Spiral interplanetary field, while STE-D points in the opposite direction. This requires different arrangements on the two observatories. Figure 9 shows the two-part STE's combined field of view. The STE units include doors to protect the detectors from exposure to sunlight during spacecraft commissioning and reorientations, and from possible contamination during thruster firings. The doors also contain thin film radiation sources that are available for calibrating the STE sensors throughout the STEREO mission.

Although STE uses a new detector design, the previous measurements in this electron energy range made with the less sensitive traditional solid state detector telescopes on the WIND spacecraft demonstrated the concept and the value of the measurement. Figure 2 
showed in situ measurements of an ICME from Larson et al. (1997) in which electrons in the STE energy range are detected within the ejecta identified with the other plasma and magnetic field measurements. These were interpreted as resulting from local magnetic field connections to a flaring active region at the time of the ejecta arrival at the WIND spacecraft. The velocity dispersion of these electrons was used to estimate the length of the field lines in the ejecta. STE measurements are intended to allow such magnetic topology and connectivity analyses routinely.

STE requires on-orbit commanding only to open and close its doors as desired. These doors are generally expected to remain open after commissioning, except perhaps during times of STEREO off-axis pointing maneuvers and major thruster firings. STE's nominal time resolution for electron fluxes and spectra is $10 \mathrm{~s}$. Like SWEA and MAG, STE participates in the IMPACT burst mode operations described below, providing $2 \mathrm{~s}$ resolution data for 10 min periods around triggering events.

\subsubsection{MAG (Magnetometer)}

Figure 13 shows the IMPACT magnetometer, MAG. MAG is a triaxial fluxgate Magnetometer built at Goddard Space Flight Center to measure the vector magnetic field in two ranges up to $65,536 \mathrm{nT}$ and up to $500 \mathrm{nT}$ with $0.1 \mathrm{nT}$ accuracy. It is a high-heritage instrument, with updated features based on the MESSENGER magnetometer design. The location of the magnetometer near the end of the $\sim 5.8 \mathrm{~m}$ IMPACT boom (see Fig. 8a) minimizes the contribution of any spacecraft fields to the measurements. In addition, a magnetics cleanliness program was enforced from the beginning of the project in order to provide a minimum cost, intrinsically clean spacecraft from a magnetics perspective. This was achieved by sensitizing instrument and spacecraft providers to materials uses and design and construction practices, by screening particularly troublesome parts such as reaction wheels, and by magnetically "sniffing" the instruments from all investigations prior to their delivery as well as the spacecraft and the integrated system. In addition, magnetic

Fig. 13 Photograph of an IMPACT MAG flight unit on its boom tray

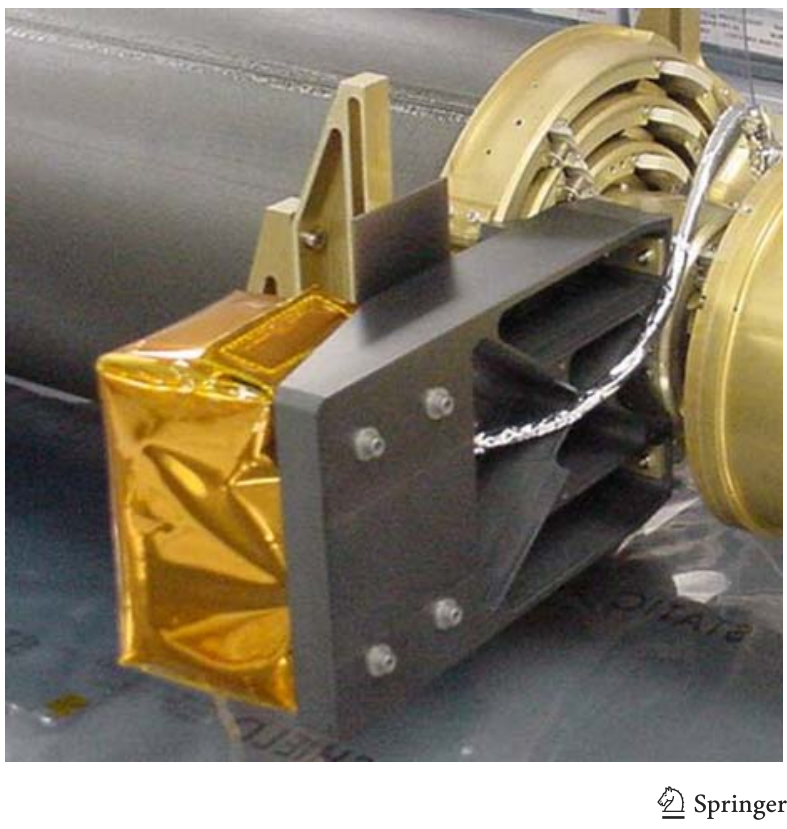


field data were obtained during integration and test phases to keep track of and characterize unavoidable signatures in the MAG data from permanent instrument and spacecraft features, and during spacecraft and instrument commissioning operations. The MAG design, calibration and operation is described in detail in a companion paper by Acuna et al. (this volume, 2007).

The digital resolution of the $500 \mathrm{nT}$ range of the IMPACT MAG is $16 \mathrm{pT}$. Signals from the fluxgate sensor are processed by the analog electronics and then fed to the IDPU for interfacing to the spacecraft. These magnetometer raw data are low pass filtered, digitized, averaged, and then formatted into a MAG telemetry packet. The magnetometer has sufficient sensitivity and dynamic range to study the magnetic fields associated with all the phases and regions of interest in the mission. For example, the lower sensitivity range permits MAG operation in the Earth's field without special shields or field cancellation equipment. The limitation of the measurements is the amount of magnetic noise introduced by the spacecraft and its instruments. The nominal accuracy of $\pm 0.1 \mathrm{nT}$ is adequate to achieve the STEREO mission science objectives for the magnetometer. The nominal time resolution of the MAG data is 8 vectors/s, though $32 \mathrm{~Hz}(\sim 0.03 \mathrm{~s})$ vector data are available during the IMPACT burst mode periods described below.

The IMPACT magnetometer is one of a long and successful line of instruments provided by GSFC for planetary, earth observing and space physics missions. The sensors and analog electronics are high performance, low noise, wide dynamic range off-the-shelf designs that have been or are being flown on numerous NASA, ESA and ISAS missions (e.g. Voyager, AMPTE, MAGSAT, GIOTTO, DMSP, WIND, CLUSTER, MGS, GEOTAIL, Lunar Prospector, Messenger). Virtually every in situ instrument on STEREO uses the MAG data (some on-board) to support their own data interpretation. Planned spacecraft rolls throughout the mission allow checks on the MAG sensor offsets and calibrations. No regular commanding is needed for MAG.

\subsection{SEP Suite}

IMPACT's SEP (Solar Energetic Particle) suite (Fig. 8c) is made up of SIT, the Suprathermal Ion Telescope, SEPT, the Solar Electron and Proton Telescope, LET, the Low Energy Telescope, and HET, the High Energy Telescope. Together, the SEP suite covers measurements of heliospheric electrons from $0.03-6 \mathrm{MeV}$, protons from $0.06-100 \mathrm{MeV}$, Helium ions from $0.12-100 \mathrm{MeV} /$ nucleon, and heavier ions from $0.03-40 \mathrm{MeV} /$ nucleon. It represents the next step in state-of-the-art measurements of SEPs carried out on ACE and WIND, which began to reveal the physics of SEP sources, acceleration, and transport through comprehensive energy range and composition measurements. A SEP-central Data Processing Unit is included as part of the LET/HET electronics box. Many of the SEP suite data handling functions are performed within SEP central, although there are also dedicated processors in SIT, and LET/HET, and the spacecraft telemetry interface is via the IMPACT IDPU-as it is for the Boom suite.

\subsubsection{SEPT (Solar Electron and Proton Telescope)}

The University of Kiel and ESA provided the SEPT (Solar Electron and Proton) telescopes to detect the dominant proton and helium ions, and the energetic electrons, of the SEP population using a two-part double-ended solid state detector design. Details on the SEPT instrument can be found in the paper by Mueller-Mellin et al. (this volume, 2007). Two doubleended telescopes in each unit, illustrated in Fig. 14, provide oppositely directed measure- 
Fig. 14 Photograph of the SEPT telescope pairs (SEPT-E and SEPT-NS) for both spacecraft, showing their aperture doors open on the left, and closed on the right

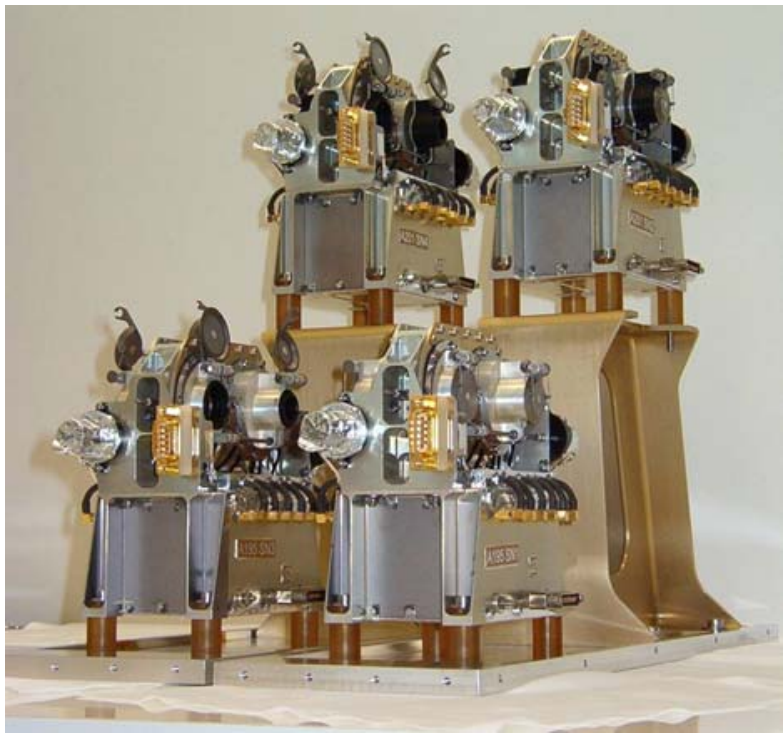

ments of both protons and alphas (fully ionized Helium ions) in the energy range 0.067.0 MeV and electrons in the energy range 30-400 keV. Separate units of double ended telescopes are mounted on the spacecraft in locations where one obtains information on particles traveling both ways along the local Parker Spiral magnetic field (SEPT-E), while the other provides the same measurements looking up and down from the ecliptic, or North and South (SEPT-NS). Their combined fields of view on each spacecraft can be seen in Fig. 9.

SEPT employs ion-implanted planar silicon detectors (PIPS) to obtain high sensitivity measurements of the particle energy while broom magnets and foils are used to separate electrons and ions. The double-ended magnet/foil design allows detection of electrons incident from the foil side with an essentially unaltered spectrum. Ions are registered when incident from the magnet side. A rare earth permanent magnet $(\mathrm{NdFeB})$ sweeps away electrons below $400 \mathrm{keV}$, but leaves ions unaffected. Using a magnet system with one magnet serving two telescopes by way of two air gaps minimizes the weight, and together with the use of low noise, high performance analog electronics allows anisotropy measurements otherwise impossible within the weight constraints. Particle anisotropy information on the nonspinning spacecraft is obtained from the observations in four look directions. The geometry factor for each of the four magnet telescopes is $0.17 \mathrm{~cm}^{2} \mathrm{sr}$ and for each of the four foil telescopes $0.13 \mathrm{~cm}^{2} \mathrm{sr}$. Unobstructed view for four viewing directions along and perpendicular to the interplanetary magnetic field necessitated different locations on the Ahead and Behind STEREO spacecraft (see Fig. 8c).

The SEPT design is based on techniques for which long-standing experience exists at the University of Kiel, ESA's Research and Scientific Support Division, and the University of California, Berkeley. On SOHO, rare-earth magnets were also successfully implemented. ESA and University of California, Berkeley, designed and built the Solid State Telescope (SST) onboard the WIND spacecraft that is still in operation. SST included many elements of the SEPT design. In the area of new technologies, SEPT uses miniaturized electronics based on a Mixed Analogue/Digital Application Specific Integrated Circuit (ASIC) which has been developed for the Space Science Dept. of the European Space Agency (ESA). This circuit contains almost all of the circuitry required to process the signal coming from 
a solid state detector. A simple logarithmic compression algorithm run in the SEP Central DPU is used to reduce the telemetry bitrate requirement to $72 \mathrm{bps}$. There are no SEPT requirements on the IMPACT IDPU other than receipt of commands (less than 20) and collecting telemetry data.

SEPT provides what may be considered the most basic STEREO SEP measurements in both the STEREO beacon and research data sets. Its observations of both electrons and major ions in the primary energy ranges of SEPs provide time profiles of both impulsive and gradual events, and anisotropies indicating the approach and recession of the ICME shock source of SEPs, as well as isotropization at the ICME shock passage. The interpretations of the SEP ion composition data from SIT and LET will depend on comparative analysis with SEPT observations.

\subsubsection{SIT (Suprathermal Ion Telescope)}

SIT is a time-of-flight (TOF) ion mass spectrometer included in IMPACT to measure ion composition in the SEP low energy (suprathermal) range. Details about SIT's design, construction and calibration at the University of Maryland, Max-Planck Institute for Solar System studies in Lindau, and Goddard Space Flight Center are given in the accompanying paper by Mason et al. (this volume, 2007). SIT measures the elemental composition of HeFe ions from $\sim 30 \mathrm{keV} /$ nucleon to $2 \mathrm{MeV} /$ nucleon, thus covering the energy range between the PLASTIC Plasma Ion Analyzer (see Galvin et al., this volume, 2007) and the IMPACT Low Energy Telescope (LET). The isotopes ${ }^{3} \mathrm{He}$ and ${ }^{4} \mathrm{He}$ are well resolved by SIT, allowing clear identification of ${ }^{3} \mathrm{He}$-rich impulsive solar particle events, critical for distinguishing between flare and interplanetary shock sources. One of the SIT instruments is shown in Fig. 15, where the telescope box (aperture cover closed) is visible on top of the amber high voltage power supply box and the electronics box at the bottom.

Ions enter the SIT telescope through two thin entrance foils (1000 $\AA \mathrm{Ni})$ and stop in the solid state detector. Secondary electrons from the entrance foil are accelerated, and deflected so as to strike chevron microchannel plates (MCPs), providing a START signal for the timeof-flight measurement. The STOP signal is obtained from secondary electrons emitted when the ion strikes the solid state detector. The solid state detector signal also provides a measurement of the kinetic energy of the ion. The time-of-flight $T$, the energy $E$, and the known path length in the telescope $L$ are then combined to derive the mass of the ion: $M=2 E(T / L)^{2}$.

Fig. 15 Photograph of the SIT flight unit for the Ahead spacecraft

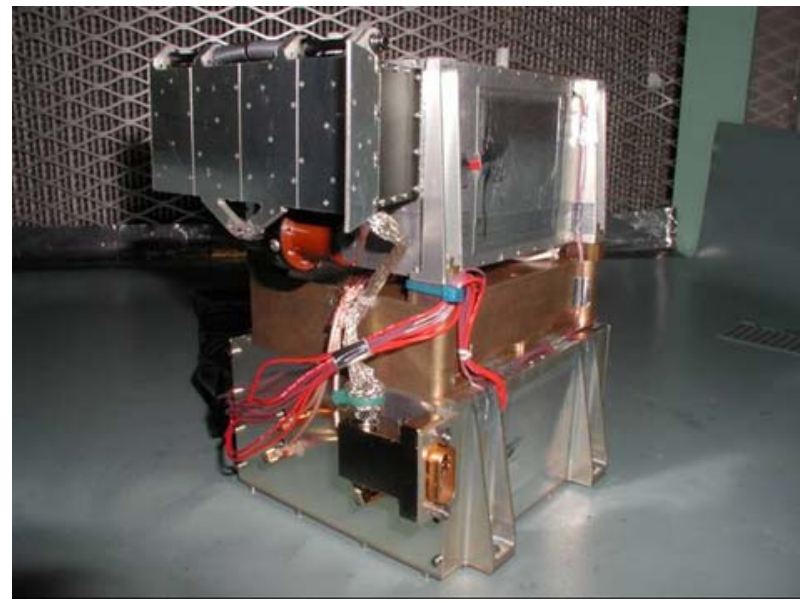


The incident energy is obtained by correcting for the energy losses in the entrance foil and detector window. The MCP and detector areas are each $6.0 \mathrm{~cm}^{2}$; the solid state detector is a silicon ion implant detector with 500 micron active thickness.

The SIT geometry factor is $0.29 \mathrm{~cm}^{2} \mathrm{sr}$, and thus large enough to allow study of even small impulsive solar particle events. For each ion triggering the telescope, time-of-flight and energy information are obtained. Full information on selected events is telemetered, and all events are classified into mass and energy bins which are summed on-board to allow rapid accumulation of intensities and spectra. The on-board analysis is done within SIT using a MISC processor. SIT uses the common SEP low-voltage power supply, obtains detector bias from the SEP Central DPU element, and generates its own high voltage $(\sim 3.5 \mathrm{kV})$ for the microchannel plate/secondary electron assembly. The SIT telescope and electronics are located on the spacecraft body near the LET, HET and SEP Central boxes (see Fig. 8c). The $\sim 44^{\circ}$ field of view of the telescope, illustrated in Fig. 9, is pointed at a small angle to the nominal interplanetary field direction, with its center at about $60^{\circ}$ to the solar direction. A sunshade prevents solar UV from striking the telescope entrance foil. A mechanical door protected the foil from acoustic damage during launch.

The SIT plays a key role in the IMPACT SEP Suite. Many impulsive solar particle events produce enhancements in the sub-MeV/nucleon range that identify connection to active regions at the Sun. During ICME passages, ${ }^{3} \mathrm{He}$-rich flare events have been observed along with impulsive suprathermal electrons, implying that at least one foot of the local magnetic field remains at least intermittently attached to the corona on those occasions (Mazur et al. 1998). SIT also provides important information in the interpretation of observations by the IMPACT Solar Electron Proton Telescope (SEPT, described above). Because SEPT counts all ions, protons usually dominate the signal, but in instances of steep energy spectra, He and heavier ions can dominate. SIT will allow the proper interpretation. Moreover, by measuring low energy ion spectra and composition at two points along a shock with STEREO, SIT provides insights into the role of suprathermal seed particles in the shock acceleration of SEPs.

Forerunners of SIT include the LICA instrument on SAMPEX, the EPACT/STEP instrument on WIND, and the ULEIS instrument on ACE. Each of these sensors was developed and constructed at the University of Maryland. The SIT telescope design is identical to EPACT/STEP. The TOF digitizing circuit was newly developed by MPS for IMPACT. GSFC collaborated on many aspects of SIT development and testing including mechanical and thermal design, assembly and integration.

SIT was calibrated with laboratory alpha-sources, and the engineering model telescope and flight electronics were tested with heavy ion beams at the Brookhaven Tandem van de Graaff accelerator facility. Post launch, instrument calibration is easily monitored from the appearance of the time-of-flight vs. energy data. If drifts do occur, the instrument's internal mass binning tables can be modified by ground command. After initial turn-on, the SIT aperture cover was opened, but SIT requires no subsequent routine commanding other than occasional (every few months) adjustment of microchannel plate bias voltages.

\subsubsection{LET (Low Energy Telescope)}

Caltech and the Jet Propulsion Laboratory provided IMPACT's LET (Low Energy Telescope) to obtain high time-resolution measurements of the composition and energy spectra of heavy ions in solar energetic particle events and in interplanetary space. LET is a novel dual directional fan-like solid state detector telescope, with ion composition capability in 
the energy range above that of SIT. It uses a $\mathrm{d} E / \mathrm{d} x$ vs. $E$ telescope configuration to provide measurements of elements from He to $\mathrm{Ni}(2 \leq Z \leq 28)$ over the energy from $\sim 2.0$ to $\sim 40 \mathrm{MeV} / \mathrm{n}$ (depending on species) and $\mathrm{H}$ over a limited range from $\sim 2$ to $\sim 13 \mathrm{MeV}$. LET also measures trans-iron nuclei with $30<Z<83$. A more detailed description of LET can be found in the accompanying paper by Mewaldt et al. (this volume, 2007).

Previous SEP studies have demonstrated that both the elemental and isotopic composition of SEPs vary significantly from event to event and composition signatures such as ${ }^{3} \mathrm{He} /{ }^{4} \mathrm{He}$, $\mathrm{He} / \mathrm{H}, \mathrm{Fe} / \mathrm{O}, \mathrm{Ne} / \mathrm{O}$ and $\mathrm{e}^{-} / \mathrm{p}$ have become the most common way in which the flare-related impulsive events and CME-driven shock acceleration events are identified. LET will make accurate composition measurements in the critical 2 to $40 \mathrm{MeV} /$ nuc energy range. This energy interval is also low enough to include a large number of solar events, but not so low that velocity dispersion washes out the time structure of acceleration effects near the Sun. The combination of SIT (described above) and LET, and also HET (described below) give complete coverage from $\sim 0.05$ to $\sim 100 \mathrm{MeV} / \mathrm{n}$ of many species, providing important tests of acceleration models. Real-time space-weather data from LET are of special interest for identifying heavy-ion rich solar events that extend to high energy ( $>50 \mathrm{MeV} / \mathrm{nuc}$ ) because Fe and other highly ionizing nuclei are capable of creating single-event upsets (SEUs) in critical spacecraft hardware. During solar active periods fluxes and energy spectra of more than a dozen species are available from LET on a continuous basis.

The LET, shown in Fig. 16, uses a fan-like array of 10 thin devices in combination with a four-detector central stack. All detectors are ion-implanted devices for high reliability and

Fig. 16 Photograph of a LET and HET flight unit, together with a SEP Central box. LET has the wide-field telescope head at the top of the picture. HET is the smaller cylindrical telescope in the middle left of the LET platform column

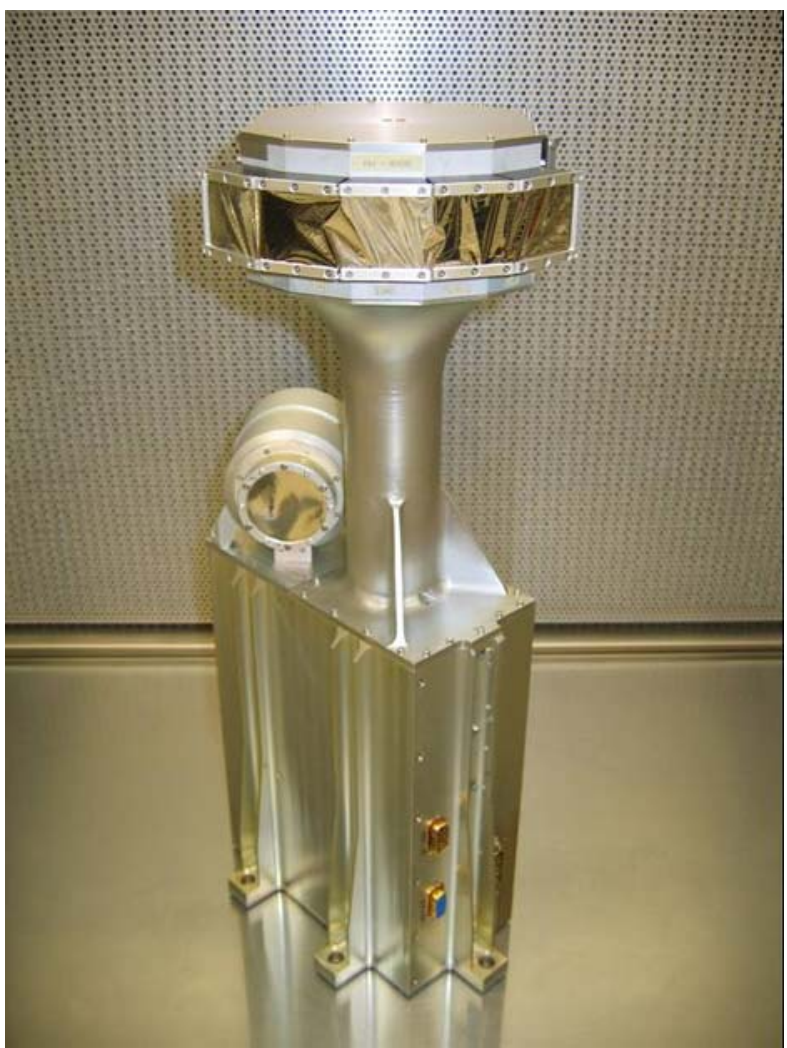


low leakage current. The symmetry axis of the telescope is aligned along the average Parker spiral ( $\sim 45^{\circ}$ from the solar direction). Five detectors arrayed at each end combine to cover $\sim 150$ deg in each direction in the ecliptic plane looking sunward and antisunward. LET is mounted on the spacecraft so that the array of detectors has an unobstructed field of view, illustrated in Fig. 9. The multi-element double-ended front end achieves a geometric factor of $\sim 4.0 \mathrm{~cm}^{2}$-sr, while providing particle anisotropy measurements over $\sim 260 \mathrm{deg}$ of the ecliptic plane.

During large solar particle events the detection rate can approach 1000 nuclei per second, far more than can be handled by the bit-rate allocation to LET if all measured pulse heights were telemetered. However, on-board processing algorithms enable the atomic number and energy of each of these events to be identified and accumulated in a $Z$ (mass) $\times E$ (energy) matrix that is telemetered every minute. This approach, which has been successfully employed in the LEMT instrument on WIND, makes it possible to achieve excellent statistical accuracy for studying time variations of SEP ion spectra and composition.

The particle detection and instrumentation approaches are based on a long series of successful energetic particle experiments flown on OGO, IMP, Pioneer, Helios, Voyager, ISEE3, SAMPEX, WIND, and ACE by the Goddard and Caltech groups and their collaborators. LET uses detector designs derived from experience on ISEE-3 and WIND, and low-power front-end electronics based on designs developed for three instruments on ACE (Stone et al. 1998a, 1998b). LET also exploits designs and on-board particle identification techniques developed for WIND/LEMT (von Rosenvinge et al. 1995).

LET requires only infrequent commanding to update on-board algorithms and possibly adjust thresholds. An average data rate of $577 \mathrm{bps}$ includes 1-minute average count rates of 16 species in $\sim 12$ energy intervals, pitch angle distributions for key species, count rates of individual detectors, and housekeeping data.

\subsubsection{HET (High Energy Telescope)}

The High-Energy Telescope (HET) provided by GSFC and Caltech is designed to make key measurements of the intensity and energy spectra of the higher energy $\mathrm{H}$ and $\mathrm{He}$ ions, and electrons accelerated in solar energetic particle events. Together with SEPT, SIT, and LET, HET will provide IMPACT solar particle energy spectra over three decades of energy. In addition, HET provides measurements of the elemental composition of $Z>2$ nuclei with energies $>30 \mathrm{MeV} / \mathrm{n}$, thereby extending the energy range of LET for key species. HET uses an all-solid-state design, and low-power electronics to achieve its objectives with minimal weight and power resources. On-board analysis algorithms provide real-time fluxes of key species important to the STEREO beacon applications, including protons and $\mathrm{He}$ with energies of $\sim 13$ to $\sim 100 \mathrm{MeV} / \mathrm{n}$, and electrons with energies from 1 to $6 \mathrm{MeV}$. Further details about HET can be found in the companion paper by von Rosenvinge et al. (this volume, 2007).

HET, shown together with LET in Fig. 16, includes two thick detectors on one end of a traditional telescope design to define the geometry of incident particles. The subsequent elements of the detector stack are made by combining thinner devices. Particles incident on the aperture which stop are identified using standard $\mathrm{d} E / \mathrm{d} x$ total energy analysis. This includes protons and $\mathrm{He}$ with $\sim 13$ to $\sim 50 \mathrm{MeV} / \mathrm{n}$. Higher energy $\mathrm{H}$ and He that penetrate can be identified using multiple $\mathrm{d} E / \mathrm{d} x$ measurements up to $\sim 100 \mathrm{MeV} / \mathrm{n}$. Measurements of ${ }^{3} \mathrm{He}$ extend from $\sim 15$ to $\sim 60 \mathrm{MeV} / \mathrm{n}$. Although HET's response to electrons is more complex than its response to ions because of the effects of electron scattering, experience with similar telescopes (flown on IMP-7,8, Pioneer 10,11, Voyager 1,2 and SAMPEX) has 
shown that HET will provide accurate measurements of the intensity and energy spectra of $\sim 1$ to $\sim 6 \mathrm{MeV}$ electrons during both solar particle events and at quiet times dominated by interplanetary Jovian electrons.

During SEP events the telemetry allocation to HET allows transmission of pulse-height data for only a small fraction of the particles triggering HET. As for LET, on-board processing algorithms are used to identify the charge and energy of the detected particles which are accumulated in a species vs. energy matrix that is telemetered once per minute. The design and operating principles of HET draw extensively on previous instruments of similar design flown by the Caltech and Goddard groups on missions that include IMP, Pioneer, Voyager, ISEE, SAMPEX and WIND. HET's detector designs are derived from these experiences and its low-power front-end electronics are based on designs developed for three instruments on ACE (Stone et al. 1998a, 1998b). Like LET, HET utilizes the on-board particle identification approaches developed for WIND (von Rosenvinge et al. 1995).

HET is mounted with its axis in the plane of the ecliptic, pointed upstream along the average Parker spiral interplanetary field (see Fig. 8c). Its field of view is compared with those of the other IMPACT SEP instruments in Fig. 9. The gain, offset, and energy conversion factors (MeV/channel) of all HET detectors were calibrated on the bench prior to launch. The response tracks of $\mathrm{H}, \mathrm{He}$, and other nuclei were also calculated using the measured detector thicknesses. As for LET and SIT, the final calibrations are performed in-flight by observing the response tracks of quiet-time and SEP particles. All parameters in the on-board identification scheme can be adjusted by command.

Like LET, HET is only infrequently commanded to update on-board algorithms and possibly adjust thresholds. The HET instrument requires an average data rate of $209 \mathrm{bps}$ This includes 1-minute average count rates of protons, He, and electrons in several energy intervals, samples of the pulse height data from individual events, once per minute dumps of the $Z \times E$ matrix, coincidence count rates, singles rates of individual detectors, and housekeeping data. HET also contributes 1-minute average fluxes of the most energetic SEP H, $\mathrm{He}, \mathrm{CNO}, \mathrm{Fe}$ ions and electrons, calculated on-board, to the real-time STEREO beacon data stream described below.

\subsection{IDPU}

IMPACT's Instrument Data Processing Unit or IDPU, shown in Fig. 17a, provides a single point digital interface to the spacecraft for all of the IMPACT instruments. It also serves the PLASTIC investigation with data processing and packaging services, and shares data with the SWAVES investigation to allow synchronized high data rate burst-mode operations (described below) following on-board identification of predefined triggering events in the IMPACT data. The IDPU is designed to manage instrument control and monitoring, the instrument timing clock, and instrument data collection, compression, and packetization for telemetry. MAG and STE-U analog interfaces also reside in the IDPU box while the other IMPACT instruments have their front-end electronics co-located with the instrument. Figure 17b shows a flow diagram of the IMPACT IDPU interfaces. Note that the SEP Suite instruments (SEPT, SIT, HET, and LET) interface with a SEP Common electronics box (SEP Central) that provides the IDPU with a single interface to the SEP suite. The IDPU is mounted inside the spacecraft body.

The IDPU occupies a small box that contains a low voltage power supply (LVPS), a MAG heater power supply, the MAG analog electronics board, a STE-U Interface board, and the Data Controller Board (DCB). The DCB contains an 80CRH196KD microprocessor, 3 Mbyte RAM, 256 Kbyte EEPROM and 8 Kbyte PROM memory, 1553 interface, and five 


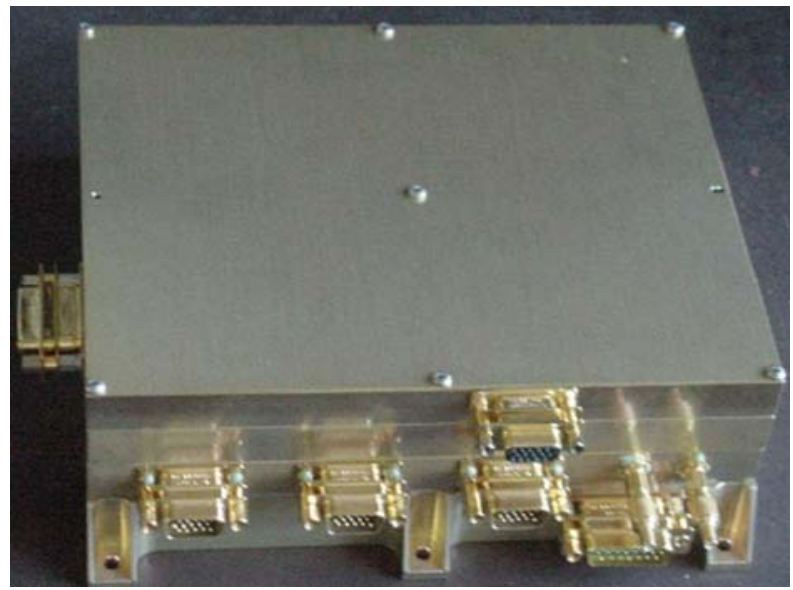

(a)

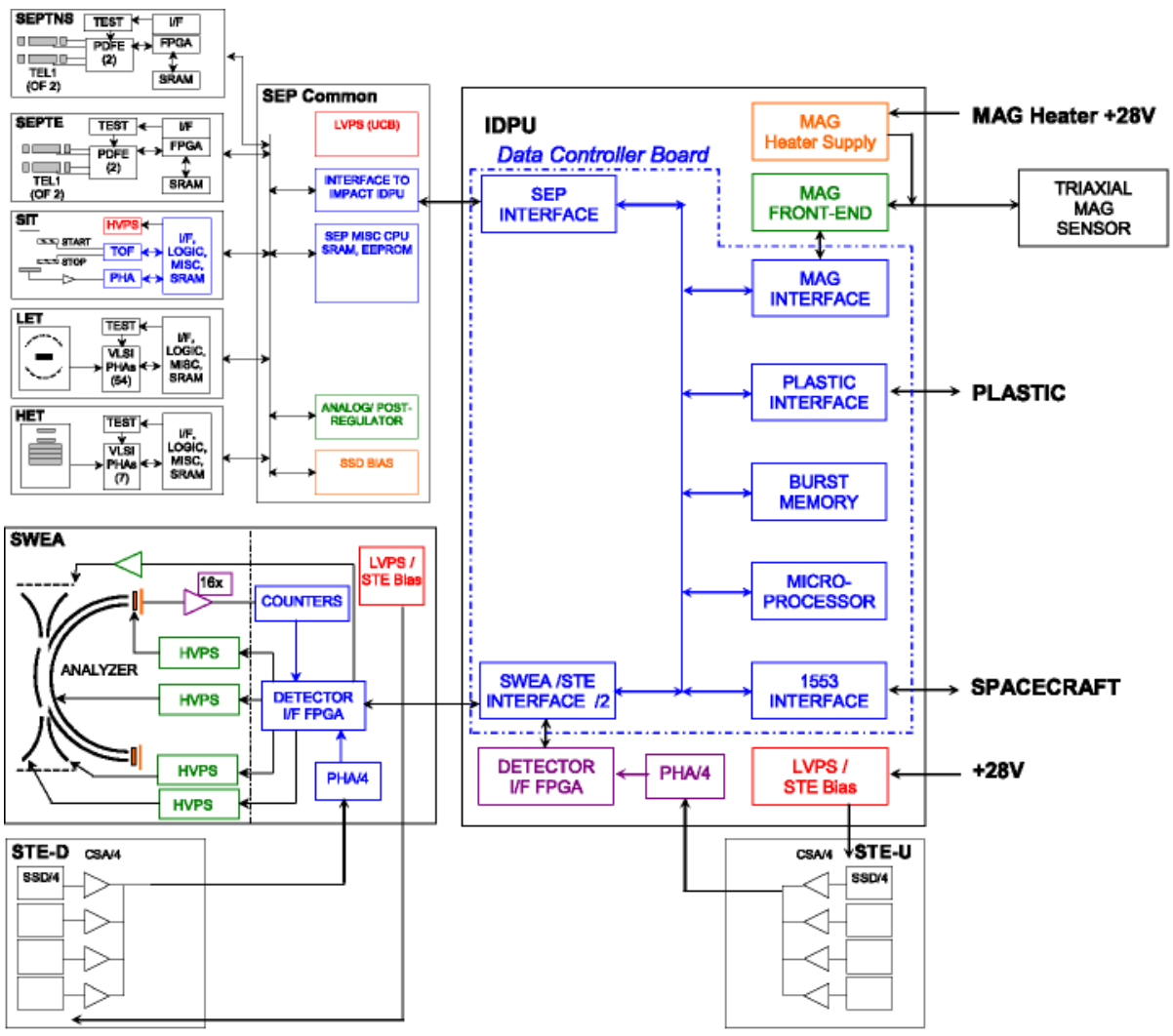

(b)

Fig. 17 a Photograph of an IDPU flight unit. The IDPU is mounted inside of the spacecraft. b Flow diagram showing the IDPU interfaces with the IMPACT instruments 
identical serial instrument interfaces. The 1553 interface connects to the Spacecraft bus for commands and telemetry. Serial instrument interfaces connect the IDPU to the IMPACT instrument suite (MAG, SEP, SWEA/STE-D, STE-U), and to PLASTIC, providing commands, timing, and telemetry. The DCB PROM contains boot code which can load flight code from either the EEPROM or from the spacecraft interface (in the event of EEPROM failure or corruption). The EEPROM contains 4 copies of the flight code. The RAM is used for code, variables, and buffers. The majority of the DCB RAM is used for a burst memory to save high time resolution interval snapshots from MAG, SWEA, and STE, which are then played out slowly via telemetry. The Burst System captures interesting events based on criteria extracted from the IMPACT instrument data streams, plus SWAVES and PLASTIC instruments. Ten minute segments of high time resolution data are monitored onboard for the "best" event of interest within three hour intervals. SWAVES and IMPACT exchange burst criteria via the 1553 bus so that burst data capture can be coordinated on board. The telemetered burst data for every three hours is then the saved high resolution data in the 10 minutes centered on the best triggering event. The triggering criteria can be changed by commanding from the ground.

The IDPU LVPS provides power to the DCB, MAG, and STE-U. The other instruments are powered separately by the spacecraft. In addition to analog and digital supply voltages, the IDPU LVPS provides a programmable bias supply $(0-150 \mathrm{~V})$ for the STE-U detectors. The MAG heater supply provides AC power to the MAG sensor heater to maintain its temperature. AC power is required to avoid DC magnetic fields at the MAG sensor. The supply provides a $50 \mathrm{kHz}$ pulse-width modulated supply controlled by a feedback loop using a temperature sensor in the MAG sensor. The MAG heater supply is independently powered by the spacecraft and functions independent of instrument power.

The IDPU was designed and constructed at the University of California, Berkeley, with the exception of the magnetometer front-end electronics, which were designed and fabricated by GSFC. It is conceptually identical to similar units successfully flown by UCB on previous spacecraft, such as the FAST SMEX mission. As on FAST, the system provides interfaces to diverse instrument types. Bringing all instruments to a common IDPU saves both instrument and spacecraft bus resources and complexity (only a single interface to the instrument complement). It also allows a more flexible sharing of instrument resources such as bit-rate, and enables collaborative science such as the coordinated burst data acquisition and shared/synchronized IMPACT and PLASTIC data sets. The common part of the IDPU was designed to be especially reliable and robust, with individual subsystems electrically isolated so that a failure in one does not impact the remainder.

Table 2 summarizes the properties and spacecraft resource requirements for the IMPACT investigation, including the IDPU, while Figs. 18a-c illustrate the overall scope of the IMPACT investigation particles measurements.

IMPACT, together with the PLASTIC ion composition and SWAVES plasma waves investigations, satisfies STEREO in situ measurement goals. The basic IMPACT data products, including the real time beacon data, are covered in a later section together with details about the IMPACT operations.

\section{IMPACT Operations and Data Plans}

\subsection{IMPACT Payload Operations Center}

STEREO mission operations are conducted from the MOC (Mission Operations Center) at APL (see Kaiser et al. and Thompson et al., this volume, 2007). However, the MOC receives 
Table 2 IMPACT instrument resource requirements

\begin{tabular}{llll}
\hline Instrument & Mass & Power & Data rate \\
\hline SEP: & & & \\
LET & $1.32 \mathrm{~kg}^{\mathrm{e}}$ & $1.18 \mathrm{~W}$ & $577 \mathrm{bps}^{\mathrm{g}}$ \\
HET & $0.60 \mathrm{~kg}$ & $0.36 \mathrm{~W}$ & $218 \mathrm{bps}^{\mathrm{g}}$ \\
SEP central & $2.19 \mathrm{~kg}$ & $4.06 \mathrm{~W}$ & $9 \mathrm{bps}^{\mathrm{h}}$ \\
SIT & $1.63 \mathrm{~kg}$ & $1.65 \mathrm{~W}$ & $424 \mathrm{bps}^{\mathrm{g}}$ \\
SEPT-E & $0.80 \mathrm{~kg}$ & $0.60 \mathrm{~W}$ & $40 \mathrm{bps}^{\mathrm{g}}$ \\
SEPT-NS & $1.18 \mathrm{~kg}$ & $0.60 \mathrm{~W}$ & $40 \mathrm{bps}^{\mathrm{g}}$ \\
Boom: & & & \\
Boom & $11.25 \mathrm{~kg}$ & & $398 \mathrm{bps}^{\mathrm{a}}$ \\
MAG sensor & $0.27 \mathrm{~kg}$ & $0 \mathrm{~W}$ & $534 \mathrm{bps}^{\mathrm{b}}+230 \mathrm{bps}^{\mathrm{c}}$ \\
SWEA/STE-D & $1.97 \mathrm{~kg}$ & $3.81 \mathrm{~W}$ & $697 \mathrm{bps}^{\mathrm{d}}$ \\
STE-U & $0.40 \mathrm{~kg}$ & $0.21 \mathrm{~W}$ & \\
IDPU: & $1.90 \mathrm{~kg}$ & $5.67 \mathrm{~W}$ & \\
Harness: & $1.34 \mathrm{~kg}$ & & $3200 \mathrm{bps}^{\mathrm{a}}$ \\
Operational heaters: & & $5.30 \mathrm{~W}$ & \\
Blankets: & $2.40 \mathrm{~kg}$ & $23 \mathrm{~W}$ & \\
Total & $27 \mathrm{~kg}$ &
\end{tabular}

${ }^{a}$ Excluding electronics in IDPU

bSWEA

${ }^{\mathrm{c}} \mathrm{STE}-\mathrm{U}+\mathrm{STE}-\mathrm{D}$

${ }^{\mathrm{d}}$ Bitrate includes Housekeeping + Burst + Beacon

e Includes $0.46 \mathrm{~kg}$ for LET mounting bracket

${ }^{\mathrm{f}}$ Includes $0.38 \mathrm{~kg}$ for SEPT-NS mounting bracket

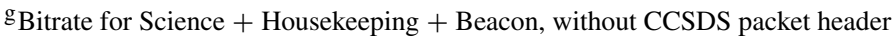

${ }^{\mathrm{h}}$ Bitrate for Housekeeping + Beacon, without CCSDS packet header

instrument commands from distributed investigation POCs (Payload Operation Centers) for upload to the spacecraft, and also distributes the Level Zero spacecraft data obtained from the Deep Space Network to the SSC (STEREO Science Center, discussed in this volume by Thompson et al., this volume, 2007) and the POCs. The IMPACT Payload Operations Center (POC) is located at the Space Sciences Laboratory at UCB. A second backup POC is located at the APL MOC in the event of a network failure at UCB.

\subsubsection{Security}

Both the UCB and APL IMPACT POCs are located in secure locations and are heavily firewalled to prevent misuse or accidental interference. The UCB POC is located in SSL's Mission Operations Center which currently supports secure operations for several missions including RHESSI and FAST.

The UCB Mission Operations Center has a dedicated backup generator in the event of a power failure. The diesel generator system delivers power to the Center within 10 seconds of power loss. Each IMPACT POC computer is connected to an uninterruptible power supply 
Fig. 18 a Nominal

interplanetary electron spectrum, showing how IMPACT

measurements cover a large energy range, including thermal, suprathermal and energetic components. b Energy and species coverage of all of the in situ particle instruments on STEREO, showing the IMPACT contributions to both ion and electron coverage. Colors indicate protons (blue), electrons (red) and heavier ions (green). The PLASTIC investigation is only roughly indicated by the blue line (solar wind proton and alpha spectrum) and blue bar at plasma energies. Details of its broader energy and ion composition coverage are described by Galvin et al. (this volume, 2007). c IMPACT SEP suite instrument coverage of suprathermal and energetic ion composition. Here the colors indicate the instrument: SIT (black), LET (blue) and HET (red). SEPT (green) measures protons and alphas only

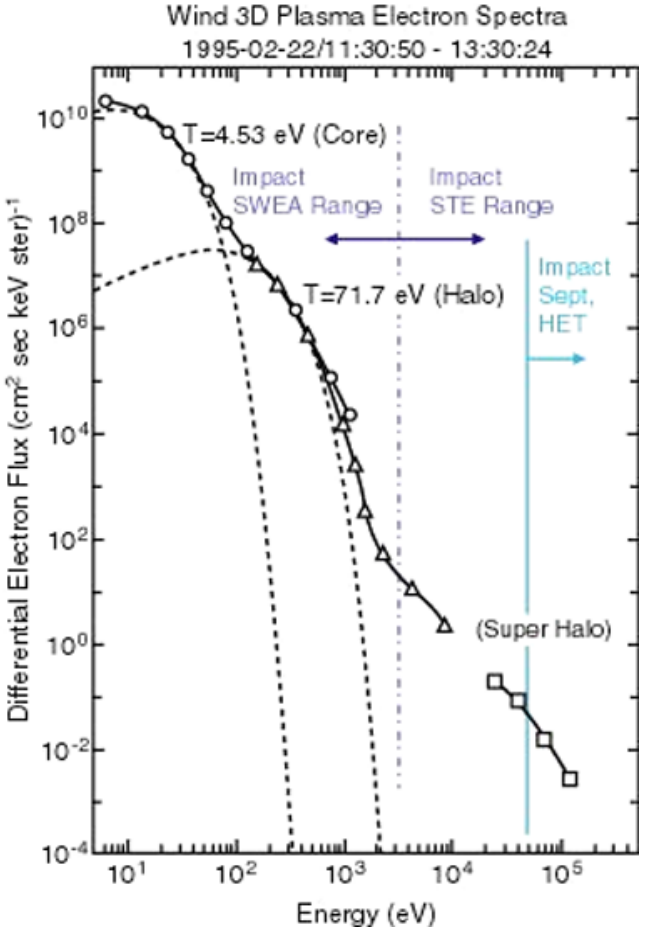

(a)

(UPS) to provide power during the potential 10 second gap in power. The diesel generator and UPS systems are tested twice monthly. Enough fuel for 24 hours of diesel generator operation is located onsite at all times.

\subsubsection{Design of POC System}

POC Operations System The IMPACT POC is an enhanced version of the existing IMPACT Command and Telemetry GSE which was used extensively in instrument testing and in Observatory I\&T. The POC consists of two Windows XP, PC-based systems, one for each spacecraft, connected to the MOC over a TCP/IP network connection through secure shell (SSH) tunneling. It is capable of generating IMPACT commands and monitoring IMPACT's state of health using the real-time telemetry stream provided by the MOC.

The POC uses a system of command mnemonics that are translated into Supplemented Command Messages (SCMs) and forwarded to the MOC for uplink. The software is capable of encoding time-tagged commands and can run command scripts written in a subset of STOL. SCMs developed by the SEP team can be saved and forwarded to the MOC after being filtered for potentially hazardous commands and approved by the local operator at the POC. The software uses a closed-loop system for verifying command receipt by the IMPACT IDPU. Commands are verified by the IDPU via instrument housekeeping and noted by the POC software. A log of all commands sent to the MOC is maintained by the software.

The POC software collects, decodes, displays and performs limit checks on the instrument state of health telemetry. The POC automatically notifies the POC operator if it detects a limit violation or other error. A log of these limit violations and errors is also maintained. 


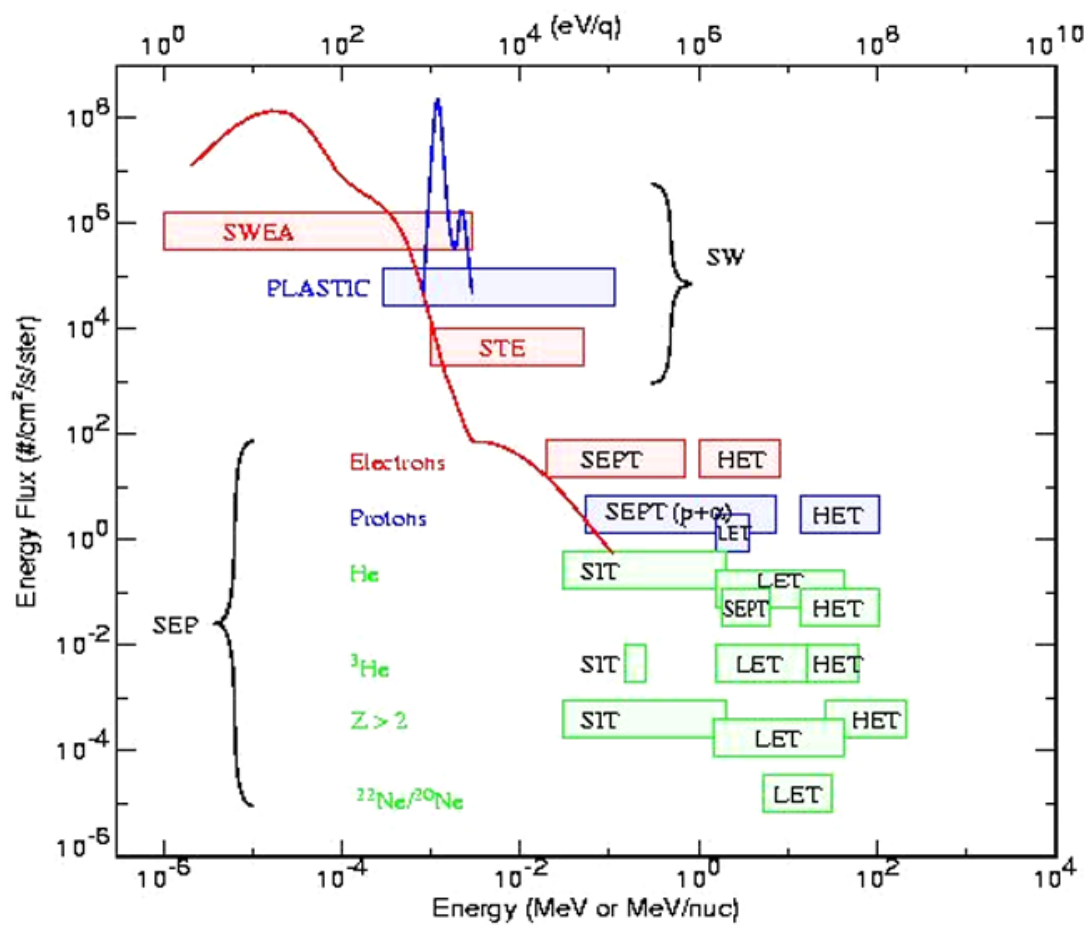

(b)

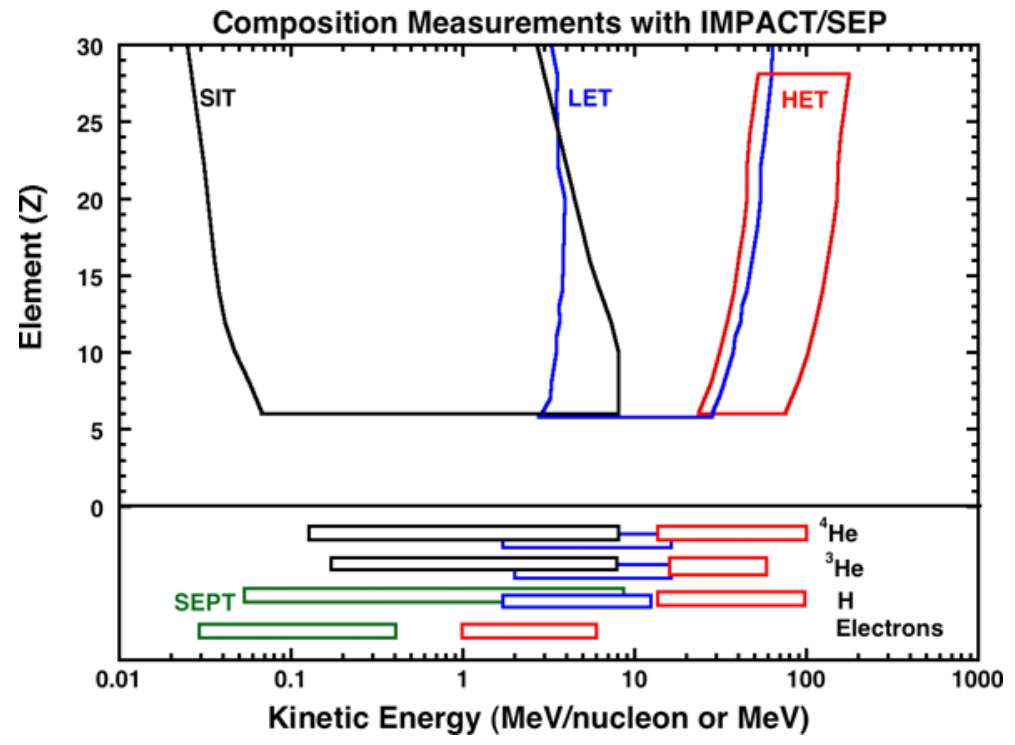

(c)

Fig. 18 (Continued) 
Real-time telemetry is saved to a local archive. The POC can generate trend plots of selected telemetry points and can display periodic instrument parameter table dumps. Separate software modules for display of IMPACT science data are provided to the IMPACT team. These modules have the ability to connect to the IMPACT POC in real time over a TCP/IP connection. In this way, team members at their home institutions can both verify instrument state of health and monitor science data in real time.

POC Data System The data processing plan is designed to minimize cost, optimize data quality and enable efficient dissemination with one point of contact, the Operations/Data Manager (ODM) at UCB. P. Schroeder is the current IMPACT ODM. The instrument providers deliver specialized software to the ODM for the routine processing of the raw spacecraft data, as well as updates based on ongoing evaluations of the received data and software performance. The ODM maintains a secure website for data and software exchange, tracks versions and anomalies, and works with the STEREO project and the IMPACT team to optimize the data sets and enable their use. The main portals to the IMPACT comprehensive data set are at UCB-SSL and the SSC. SSL maintains a website with browse and download capabilities. SSL is also responsible for delivering the archive of processed IMPACT data to the SSC. Higher level, science-quality, key parameter data that includes merged 1-min resolution subsets of IMPACT, PLASTIC and SWAVES key parameter data, together with selected event data identifications and characterizations, are made available through another data server at UCLA.

Figure 19 summarizes the data flow from receipt from the STEREO MOC through to the data archive. The Level zero data are unpacked, time sorted, and distributed to the STEREO teams by the MOC. The IMPACT Level zero data are downloaded to SSL, catalogued, and distributed by the ODM to the individual IMPACT instrument teams. The instrument provider is responsible for quality checking their data and informing the ODM of any irregularities. The ODM analyzes and reports any such irregularities in either the instrument or IDPU level zero data and records them in a database to be archived with Level 1 data. The Level zero archive includes the space weather beacon data.

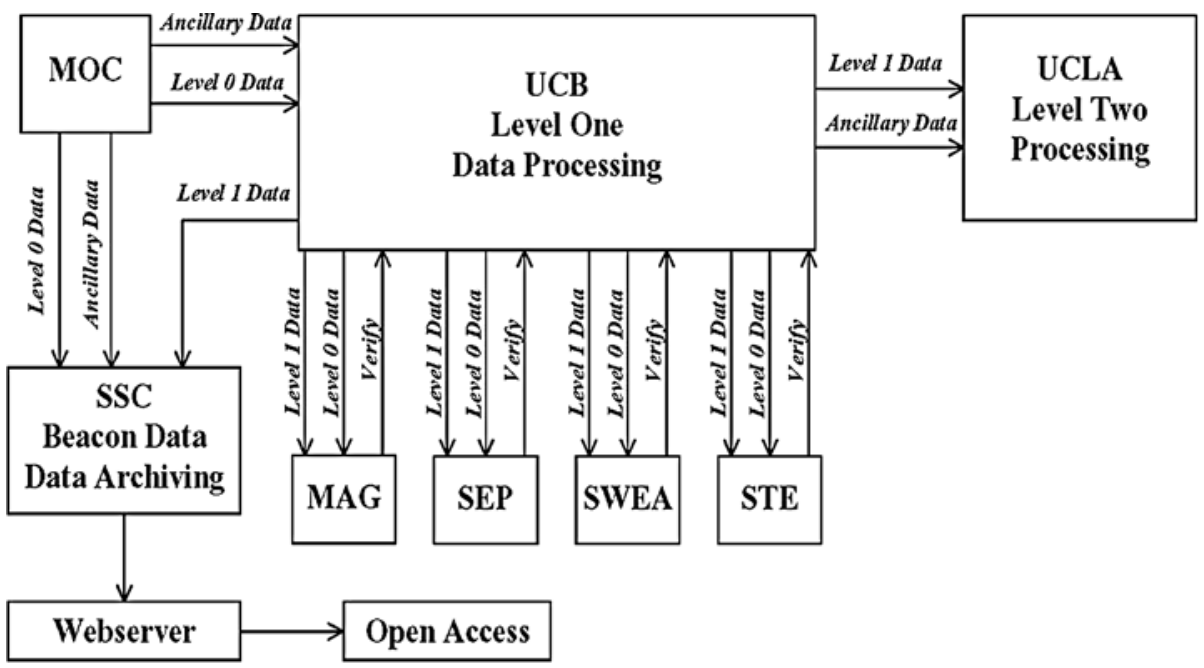

Fig. 19 Diagram of the IMPACT data processing and products flow 
Level 1 data are obtained by running the Level zero data through the calibration and IMPACT data processing software at UCB. Original versions collected by the ODM from each instrument provider prior to launch are updated as necessary by the developer. Both the Level zero and Level 1 data, together with calibration and basic processing software for the full investigation, are regularly submitted to the designated project archives at the SSC by the IMPACT ODM. These Level 1 data are also referred to as high (time) resolution data, and contain all of the measurement results available from the IMPACT instruments in physical units.

The second stage of IMPACT data processing, at an in situ STEREO data access site at UCLA, generates Level 2 and 3 products for serving and archiving. The Level 2 or key parameter data consist of synchronized one-minute summary data sets containing the most often used quantities from IMPACT, PLASTIC and SWAVES, such as plasma moments and magnetic fields. Level 3 products result from basic analyses of the in situ data that identify and characterize events such as shocks, ICMEs and SEP events.

The Level 2 processing software creates the merged, averaged data useful for much of the research involving combinations of STEREO in situ data. These summary data include all of the IMPACT and PLASTIC Beacon data contents in final, calibrated form (see IMPACT's list of Beacon data below). They also include additional derived products. Web access is provided to the Level 2 summary data through the on-line UCLA solar wind data server (http://www-ssc.igpp.ucla.edu), while specialized data products and displays can be accessed through hyperlinked instrument contributor Web sites.

The Level 3 data products are available for users interested in specific types of in situ events. Team members review the database to identify and characterize features and events of interest. These Level 3 data are also made available for the STEREO project archival database. The overall timeline for the complete data processing chain is shown in Table 3 .

While the routine processing steps are centralized to minimize costs, we emphasize that each cognizant CoI develops the calibration factors and software for the individual data sets, performs quality checks, calculates moments as appropriate and compares these with the real-time space weather data that are transmitted separately. This critical validation process is placed under cognizant CoInvestigator control, both to minimize the possibility of errors, and to maximize the utilization of experience and existing software. As soon as the data have passed the data quality checks, the ODM is notified and the data are documented and submitted to the archive. Software is included with any level of archived IMPACT data for access and display, making the IMPACT data readily usable by the scientific community.

Some minor Level 1 processing is desirable for the space weather Beacon data from IMPACT, for example the calculation of the magnetic field in various coordinate systems such as GSM and GSE, or the calculation of SEP fluxes using improved geometric factors and updated efficiencies. For this purpose, the IMPACT team supplies the necessary software to the SSC which is the distribution point for the real-time STEREO Beacon data.

Table 3 Nominal process time from release of Level zero data (location)

\begin{tabular}{ll}
\hline Check and verify Level 0, catalogue & $<3$ hours (UCB) \\
Run calibration on Level 0 and Level 1 & 24 hours (UCB) \\
Level 0 and 1 data + software to SSC archive & $1-2$ months (UCB) \\
Process Level 2 (summary database) & 2.5 months (UCLA) \\
Level 2 products to SSC and archive & 2.5 months (UCLA) \\
Create Level 3 value-added products & 3 months (UCLA) \\
Level 3 products to SSC archive & 3 months (UCLA \\
\hline
\end{tabular}




\subsubsection{Configuration Management}

Revisions to the POC operations software and hardware replacements require approval by a committee composed of the technical Project Manager, the Operations/Data Manager, any affected instrument leads and CoInvestigators as designated. Such changes to the operations system are tested and verified by this committee before implementation. A version numbering system tracks each change. Hardware configuration changes are similarly recorded in a web-accessible database.

As noted above, the IMPACT instrument teams provide software to the SSC to produce corrected Beacon data, and to UCB-SSL to process the Level 1 data and submit to the archive. Each data processing routine provided by the instrument teams to process Beacon, Level 1 Data, or Level 2 Data is associated with a software version number. Each time that a routine is revised, that routine's software version number is incremented. All original routines have a software version number of 1 . Every data product created by one of these routines includes a field for the software version number which indicates the number of the software revision used to create that product. The Level 2 Data products also include the software revision number that created the Level 1 Data product from which they are derived.

When an instrument team revises a Beacon Data, Level 1 Data, or Level 2 Data processing routine, the revised routine undergoes a review process to demonstrate that the revised routine performs correctly. During the review of a revision of Beacon Data or Level 1 Data processing software, the ODM uses the revised routine to produce data products for at least three days of data. During the revision of Level 2 processing software, UCLA personnel similarly use the revised routine to produce data products for at least three days of data. The dates that are processed are chosen for their differing characteristics (i.e. One date during an ICME event, one date during which no events occur, one date within the Earth's magnetosphere, etc.). A committee consisting of the ODM, the Co-Investigator in charge of the instrument, and ad hoc members chosen by the ODM and Co-Investigator such as instrument engineers and software developers determine the dates to be tested and whether the data products created with the revised routine are correct. If the products created are not correct, the revision is rejected. If the committee determines that the routine is functioning correctly, the ODM archives the previous version of the routine, keeping it available for retrieval by team members and the public by a web interface from UCB-SSL. The new routine replaces the previous routine in the data processing stream. Data products created by the routine are reprocessed from the beginning of the mission to the present. These data products replace the products created by the old routine. If a Level 1 processing routine is modified and new Level 1 data produced, UCLA is notified of the updated data products so that new Level 2 Data are generated.

Each IMPACT instrument calibration data file provided by the developer is also associated with a calibration version number. Every data product created using one of these calibration data files includes a field for the calibration version number which indicates the number of the calibration revision used to create that product. When an instrument team revises a calibration data file, the revised calibration data file must undergo a review process identical to that of a processing software version. The ODM, the Co-Investigator in charge of the instrument, and others chosen by the ODM and Co-Investigator such as instrument engineers and software developers will approve the revised calibration data file after testing its use on at least three days of data. A publicly accessible web page maintained at UCB by the ODM contains a history of calibration revisions. The web page indicates the latest calibration version number for each calibration data file, the dates that revisions, past and present, were approved, and notes on the revisions made to the calibration data files. 


\subsection{Phasing Orbit Operations}

During the phasing orbits (see Thompson et al., this volume, 2007), the IMPACT technical Project Manager who was responsible for the instrument development and delivery phase was in charge of IMPACT's operations with the assistance of the ODM. Routine operations were conducted from the UCB POC. During instrument commissioning, web-accessible displays were available to team members to monitor events remotely. The UCB POC was connected to the STEREO MOC's voicenet during critical IMPACT operations and events. Critical operations during the phasing orbits included:

- Day 3: Powering up of the IMPACT IDPU, SEP Central, and the MAG and SWEA instruments. The SWEA cover was opened on Day 3. These systems were then checked out for the following few days.

- Day 9: Deployment of the IMPACT boom.

- Day 19: Powering up and checking out of STE and the SEP instruments.

- Day 25: The first MAG calibration roll manoeuver. The spacecraft was rotated to calibrate MAG post-launch.

Release of the first IMPACT data products from the phasing orbits occurred approximately 2 months after launch.

\subsection{Normal Operations}

Once the STEREO spacecraft entered heliocentric orbit, the ODM became responsible for IMPACT operations. Transition of control from the Project Manager to ODM began in the late stages of the phasing orbits. The ODM has an alternate who is able to conduct IMPACT operations when the ODM is unavailable.

The ODM is responsible for the creation of IMPACT command sequences for all IMPACT instruments except the SEP suite. When commands are needed for SWEA, STE or MAG, the ODM uses the POC operations console to assemble, test, and submit commands to the MOC. When SEP commands are required, the SEP instrument providers send the ODM a full Supplemented Command Message. The ODM tests and submits these commands to the MOC. The IMPACT suite requires very few commands, on the order of three per spacecraft per day. These generally consist of minor threshold adjustments and small changes to the burst mode criteria to better catch important events.

The POC system is capable of monitoring real time telemetry without user intervention. During these unattended periods, the POC system monitors for limit violations and other errors. If a limit violation or error is detected, the ODM receives an automatically produced text page through an automated email/pager system with information about the error condition. The ODM maintains a list of responses including contact information for subsystem personnel for each error condition. If the designated subsystem personnel are unavailable when an anomaly occurs, the ODM performs a backup action that was agreed upon before launch. This list of contingency responses and contact information is also available to the ODM's alternate and is regularly updated.

As noted above, under normal operations, processed Level 1 data are expected to appear on the IMPACT data server website within $\sim 30$ days of receipt on the ground. Users who are interested in plasma microphysics may be particularly interested in the special Burst Mode observations. 
Burst Mode The IMPACT burst mode is used to capture high time resolution data during "events", and play it back slowly as part of the IMPACT telemetry system. Instrument modules provide a continuous stream of high time resolution data to the Burst System, which is in a circular RAM buffer. A criterion function based on IMPACT, PLASTIC and SWAVES data indicates when an event occurs and the event size. The best event over each consecutive three-hour interval is then sent as the next burst. This involves three buffers; one for the transmitting burst event, one for the current best burst event, and one for the current data while looking for a better burst event. Most of the 3 Mbyte RAM is used for these Burst buffers. The buffered burst data include data from $5 \mathrm{~min}$ before to $5 \mathrm{~min}$ after the sample satisfying the preprogrammed burst criteria. The time resolution of the IMPACT burst mode data is $2 \mathrm{~s}$ for SWEA and STE, and $32 \mathrm{~Hz}(\sim 0.03 \mathrm{~s})$ for MAG. Saved burst data are passed to the Burst System by the instrument modules in the form of CCSDS telemetry packets. Burst data are processed in the IDPU in parallel with the normal SWEA, STE and MAG data, so there is no interruption to the normal Level Zero data stream from IMPACT.

The data are subjected to the burst event criteria computation that is part of the Instrument software. The burst criteria are passed to the Burst System, which uses them to decide what data are kept for transmission. At the time of launch, the burst criteria were set to be a weighted sum of individual burst criteria from MAG, SWEA, STE, PLASTIC, and SWAVES:

- The MAG criteria requires sudden changes in the MAG field vector.

- The SWEA criteria requires sudden changes in the SWEA count rates around $400 \mathrm{eV}$.

- The STE criteria requires increases in the STE count rate, integrated over energy and direction.

- The PLASTIC criteria requires sudden changes in the computed solar wind velocity vector.

- The SWAVES criteria requires increases in electric field fluctuation power in selected frequency bands.

These criteria can be reprogrammed after experience is gained with the data.

\section{Data Products, Access, and Analysis Plans}

\subsection{Data Product Descriptions and Content}

\subsubsection{Level Zero}

Level Zero data from the spacecraft via DSN are provided by the STEREO MOC to the IMPACT POC via a secure ftp interface. These data consist of raw telemetry frames in a format defined by the STEREO MOC/POC/SSC ICD. The telemetry provided in Level Zero files are cleaned, merged and time-ordered by the MOC before they are sent to the POC and the SSC.

\subsubsection{Level 1}

As mentioned above, Level One data are produced at UCB-SSL with software and calibration data provided by the IMPACT instrument teams. Each IMPACT instrument has a designated individual responsible for ensuring the delivery of Level 1 processing software 
and for validating the Level 1 data products. Level 1 data are divided into instrument products and consist of all science data at highest time resolution in scientific units and, in the case of MAG data, rotated into heliographic-radial-tangent-normal coordinates (HGRTN). The Level 1 data are stored in ISTP-compliant CDF files whose structure and content as of launch are given below. Separate data files for burst mode data are produced. A webaccessible database is available to indicate burst mode time intervals. Table 4 summarize the content of IMPACT Level 1 data files.

Table 4 IMPACT Level 1 data

\begin{tabular}{|c|c|}
\hline Instrument & Quantities in Level 1 data \\
\hline \multirow[t]{3}{*}{ MAG } & Bx, By, Bz, B (all in nanoteslas) \\
\hline & $\begin{array}{l}\text { Coordinate system: Spacecraft coordinates, STEREO } \\
\text { Solar Orbital Coordinates, HGRTN }\end{array}$ \\
\hline & Time resolution: $0.1 \mathrm{~s}$ \\
\hline \multirow[t]{17}{*}{ SWEA } & $\begin{array}{l}\text { Pitch angle distributions in flux units }\left(1 / \mathrm{cm}^{2} / \mathrm{s} / \mathrm{ster} / \mathrm{eV}\right) \\
\text { including: }\end{array}$ \\
\hline & Look angles [NA] in degrees \\
\hline & Energy steps $[\mathrm{NE}]$ in eV \\
\hline & Time resolution: $10 \mathrm{~s}$ \\
\hline & $\begin{array}{l}\text { 3D electron distributions in flux units }\left(1 / \mathrm{cm}^{2} / \mathrm{s} / \mathrm{ster} / \mathrm{eV}\right) \\
\text { including: }\end{array}$ \\
\hline & Look angles [NA] in degrees \\
\hline & Energy steps $[\mathrm{NE}]$ in eV \\
\hline & $\mathrm{NE}=16, \mathrm{NA}=80$ \\
\hline & Time resolution: $30 \mathrm{~s}$ \\
\hline & Electron moments: \\
\hline & 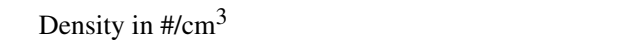 \\
\hline & Velocity in km/s \\
\hline & Pressure tensor in $\mathrm{eV} / \mathrm{cm}^{3}$ \\
\hline & Heat flux in $\mathrm{eV} / \mathrm{cm}^{2} / \mathrm{s}$ \\
\hline & SC potential in volts \\
\hline & Time resolution: $2 \mathrm{~s}$ \\
\hline & $\begin{array}{l}\text { Coordinate systems: STEREO Solar Orbital } \\
\text { Coordinates, HGRTN }\end{array}$ \\
\hline \multirow[t]{6}{*}{ STE } & Electron flux [NE, NA] in units of flux $\left(1 / \mathrm{cm}^{2} / \mathrm{s} / \mathrm{eV}\right)$ \\
\hline & Look angles [NA] in degrees \\
\hline & Energy steps $[\mathrm{NE}]$ in eV \\
\hline & $\mathrm{NE}=32, \mathrm{NA}=8$ \\
\hline & $\begin{array}{l}\text { Coordinate systems: STEREO Solar Orbital } \\
\text { Coordinates, HGRTN }\end{array}$ \\
\hline & Time resolution: $10 \mathrm{~s}$ \\
\hline \multirow[t]{3}{*}{ SEPT } & $\begin{array}{l}\text { Electron count rates (counts/s) in two look directions at } \\
32 \text { energies }\end{array}$ \\
\hline & $\begin{array}{l}\text { Ion count rates (counts/s) in two look directions at } 32 \\
\text { energies }\end{array}$ \\
\hline & Time resolution: $1 \mathrm{~min}$ \\
\hline
\end{tabular}


Table 4 (Continued)

\begin{tabular}{|c|c|}
\hline Instrument & Quantities in Level 1 data \\
\hline 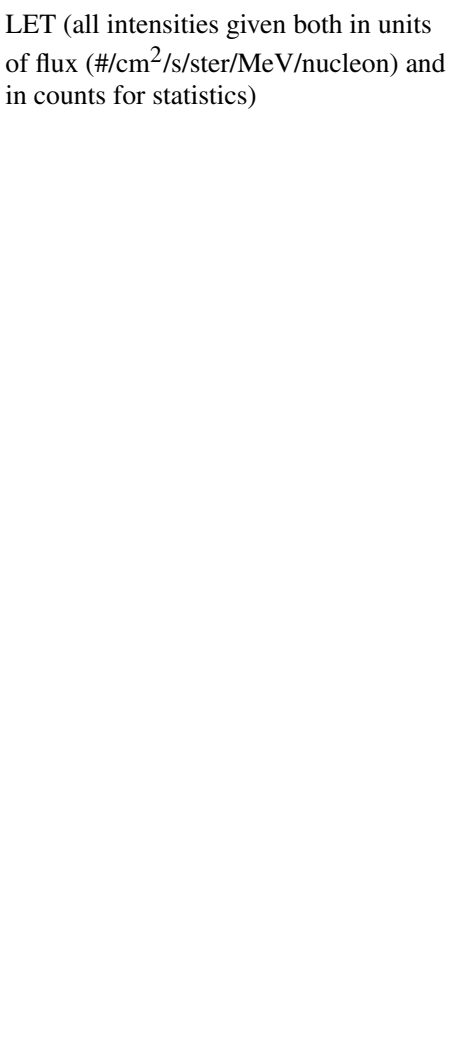 & $\begin{array}{l}\text { Unsectored data: } \\
\text { Proton intensities at } 12 \text { energies } \\
3 \mathrm{He} \text { intensities at } 11 \text { energies } \\
4 \mathrm{He} \text { intensities at } 12 \text { energies } \\
\mathrm{C} \text { intensities at } 12 \text { energies } \\
\mathrm{N} \text { intensities at } 12 \text { energies } \\
\mathrm{O} \text { intensities at } 12 \text { energies } \\
\mathrm{Ne} \text { intensities at } 13 \text { energies } \\
\mathrm{Na} \text { intensities at } 8 \text { energies } \\
\mathrm{Mg} \text { intensities at } 14 \text { energies } \\
\mathrm{Al} \text { intensities at } 9 \text { energies } \\
\mathrm{Si} \text { intensities at } 14 \text { energies } \\
\mathrm{S} \text { intensities at } 13 \text { energies } \\
\mathrm{Ar} \text { intensities at } 13 \text { energies } \\
\mathrm{Ca} \text { intensities at } 13 \text { energies } \\
\text { Fe intensities at } 16 \text { energies } \\
\mathrm{Ni} \text { intensities at } 9 \text { energies } \\
\text { Sectored data (all in } 16 \text { look directions): } \\
\text { Proton intensity at } 1 \text { energy } \\
3 \mathrm{He} \text { intensity at } 1 \text { energy } \\
4 \mathrm{He} \text { intensities at } 2 \text { energies } \\
\mathrm{CNO} \text { intensities at } 2 \text { energies } \\
\mathrm{NeMgSi} \text { intensities at } 2 \text { energies } \\
\text { Fe intensities at } 2 \text { energies } \\
\text { Time resolution: } 1 \text { min }\end{array}$ \\
\hline HET & $\begin{array}{l}\text { Much like the LET set with unsectored, high energy } \\
\text { resolution data and sectored, low energy resolution } \\
\text { data. } \\
\text { Intensities for } \mathrm{H}, 3 \mathrm{He}, 4 \mathrm{He}, \mathrm{C}, \mathrm{O}, \mathrm{Ne}, \mathrm{Mg}, \mathrm{Si} \text { and } \\
\mathrm{Fe}-\text { exact numbers of energy bins still being } \\
\text { determined as of this writing. Same units as LET } \\
\text { Time resolution: } 1 \mathrm{~min}\end{array}$ \\
\hline SIT & $\begin{array}{l}\text { Intensities for } \mathrm{H}, 3 \mathrm{He}, 4 \mathrm{He}, \mathrm{C}, \mathrm{O}, \mathrm{NeS} \text { (range } \mathrm{Ne} \\
\text { through } \mathrm{S} \text { ), and } \mathrm{Fe} \\
\text { Exact energy ranges TBD } \\
\text { Units: \#/cm } / \mathrm{s} / \mathrm{ster} / \mathrm{MeV} / \text { nucleon } \\
\text { Time resolution: } 1 \mathrm{~min}\end{array}$ \\
\hline
\end{tabular}

\subsubsection{Level 2}

Level 2 data are produced at the STEREO in situ data access site at UCLA. These in situ summary or "key parameter" data consist of a subset of IMPACT's measured quantities averaged to a synchronized one minute cadence. For several reasons, the content of the 
Beacon data, described below, was chosen as the baseline for the Level 2 data set. Quantities from the PLASTIC and SWAVES instruments are also included as well as derived quantities such as solar wind dynamic, thermal and magnetic pressures and plasma beta. The key parameter data are available in ASCII and binary flatfile formats and are calibrated, verified and publishable data products.

The beacon data quantities were selected by the STEREO IMPACT, PLASTIC and WAVES teams as concise, representative subsets of their most widely and generally used measurements. Together with the derived parameters that often depend on the results from several instruments, they are intended for wide use, statistical studies, and beacon data retrospective validations.

\subsubsection{Level 3}

Level 3 data are also produced at UCLA with assistance from various IMPACT, PLASTIC and SWAVES team members. These data include feature and event lists of scientific interest. Shock identifications are accompanied by an estimate of their properties such as strength, shock normal angle, and angle of incidence. ICME start and stop times are recorded together with any derived flux rope or other model fit parameters. SEP event start and stop times and peak fluxes are noted together with related flares and CMEs based on STEREO and other available information. They are provided in ASCII table format.

\subsubsection{Beacon Data}

Beacon data are produced at the STEREO Science Center (SSC) where final processing and correction steps are made using software provided by, and updated by, the IMPACT team. These data provide access to near realtime solar wind electron, interplanetary magnetic field, and SEP quantities particularly useful for space weather predictions and predictive modeling. The 1-min averaged IMPACT beacon data are listed in Table 2 and in more detail in Table 5. These data will reside natively in the CDF format but will be available in other formats such as ASCII.

\subsubsection{Complementary L1 In Situ Data Sets}

Complementary data sets from spacecraft located at L1 are made available in parallel with the IMPACT data at SSL. In situ products from the ACE and Wind spacecraft can be browsed and downloaded through the SSL web interfaces described below. As much as possible, data from the L1 spacecraft are tightly integrated with IMPACT's data services to enable immediate comparison and analysis of analogous data quantities.

\subsection{Data Access and Browsers}

\subsubsection{STEREO Science Center Beacon and Archive Access}

The STEREO Science Center provides access to the IMPACT Beacon data set. It also archives all IMPACT science products (see Thompson et al., this volume, 2007). Users interested in beacon data history and performance can access the IMPACT beacon data at the usual IMPACT data access sites and compare it to the IMPACT key parameter (Level 2) data at UCLA, or they can compare the two in the archived IMPACT data. 
Table 5 IMPACT Beacon Data

Beacon Data from IMPACT include the following:

MAG:

B vectors in nT, 6 samples/minute in spacecraft coordinates. Beacon Data Processing software transforms the data into other coordinate systems as well (e.g. STEREO Solar Orbital and HGRTN).

STE:

For STE-U:

Solar electron fluxes at 5 (modifiable) energies at 1 sample/minute.

Non-solar electron fluxes at 5 energies at 1 sample/minute.

For STE-D:

Electron fluxes at 5 energies at 1 sample/minute.

Units $\# / \mathrm{cm}^{2} / \mathrm{s}$

SWEA:

Moments calculated from 2 second integration once per minute. Moments include:

Electron density in cnts $/ \mathrm{cm}^{3}$

Electron bulk velocity in km/s (in STEREO Solar Orbital and HGRTN)

Electron pressure tensor in $\mathrm{eV} / \mathrm{cm}^{3}$

Electron heat flux vector in $\mathrm{eV} / \mathrm{cm}^{2} / \mathrm{s}$ (in STEREO Solar Orbital and HGRTN)

Pitch Angle Distributions (with respect to B field) at 2 (modifiable) energies in 12 look directions once per minute, units cnts $/ \mathrm{cm}^{2} / \mathrm{s}$

SEP:

SEP status

Note: For all SEP fluxes, units are $1 / \mathrm{cm}^{2}$-s-ster-MeV or $1 / \mathrm{cm}^{2}-\mathrm{s}-\mathrm{ster}-\mathrm{MeV}$-nucleon.

In addition all SEP quantities given in raw counts for statistical analysis

SEP-SEPT:

Electron flux at 2 energies in 4 look directions averaged over 1 minute.

Electron flux at 2 energies summed over 4 look directions averaged over 1 minute.

Ion flux at 2 energies in 4 look directions averaged over 1 minute.

Ion flux at 2 energies summed over 4 look directions averaged over 1 minute.

SEP-LET:

Proton flux at 1 energy in 2 look directions averaged over 1 minute.

Proton flux at 2 energies summed over all look angles averaged over 1 minute.

He flux at 2 energies in 2 look directions averaged over 1 minute.

He flux at 1 energy summed over all look angles averaged over 1 minute.

3 He flux at 2 energies summed over all look angles averaged over 1 minute.

CNO flux at 3 energies summed over all look angles averaged over 1 minute.

Fe flux at 4 energies summed over all look angles averaged over 1 minute.

\section{SEP-HET:}

Electron flux at 1 energy averaged over 1 minute.

Proton flux at 3 energies averaged over 1 minute.

He flux at 3 energies averaged over 1 minute. 
Table 5 (Continued)

CNO flux at 2 energies averaged over 1 minute.

Fe flux at 1 energy averaged over 1 minute.

SEP-SIT:

HE flux at 4 energies averaged over 1 minute.

CNO flux at 4 energies averaged over 1 minute.

Fe flux at 4 energies averaged over 1 minute.

IMPACT (in general):

Instrument status.

As an element of the overall STEREO beacon resources, UCB also hosts a browser for visualization of the in situ Beacon data, linked from the SSC website http://stereo-SSC. nascom.nasa.gov. This browser is highly customizable allowing users to plot desired quantities for a given time period. It includes Beacon data from IMPACT, PLASTIC and SWAVES.

\subsubsection{UCB Data Portal}

UCB provides several ways for visualizing and accessing IMPACT data. Routine static plots are created from a fixed subset of IMPACT, PLASTIC and SWAVES quantities in IMPACT Level 1 data and its equivalents for the other instruments. These plots are available for 1-day, 7-day, and Carrington rotation time intervals at the site http://sprg.ssl.berkeley.edu/impact/. These are not customizable by the user, and are intended as quick-look resources. Orbit plots are provided to indicate the STEREO spacecraft locations for each browse period.

UCB also provides a highly flexible interface to IMPACT Level 1 data and data from PLASTIC and SWAVES based on the CDAWeb model at http://cdaweb.gsfc.nasa.gov. This interface allows the user to specify quantities and time intervals for plotting. It also gives the user the ability to customize a plot's layout and look. Once a user has created a plot layout, the user is able to save that layout for use in future sessions. The web interface at http://sprg.ssl.berkeley.edu/impact/ is also seamlessly connected with a Level 1 data server. This server provides direct access to the Level 1 data and performs on-the-fly translation to formats other than CDF such as ASCII, HDF, and FITS. Wind and ACE data are also served through this interface for easy comparisons with their similar STEREO counterparts.

A SOAP-based application programming interface (API) is available as well. This API allows for the creation of custom applications by the user community, supporting such activities as advanced data queries by spacecraft separation and by specific content criteria.

\subsubsection{UCLA Data Portal}

UCLA provides a web portal for the Level 2 and Level 3 data sets. The UCLA digital service allows users to choose quantities for plotting and enables access to data in ASCII and binary flatfile formats. Users can go to this site at http://www_ssc.igpp.ucla.edu to obtain the synchronized 1 min key parameters from PLASTIC and SWAVES merged with IMPACT key parameter data, as well as Level 3 information such as ICME identifications and SEP event lists (see previous discussion of Level 2 and Level 3 data products). 


\subsection{Data Analysis Plans}

As noted earlier, the Helios twin spacecraft mission, a cooperative venture led by the Federal Republic of Germany during portions of solar cycle 21, is the most direct forerunner of STEREO. The timing of the Helios mission was such that images from the SMM and Solwind mission coronagraphs could be exploited to study the solar sources of the detected shocks and ICMEs. In the same spirit as STEREO and ACE, IMP-8 provided regular, complementary near-Earth measurements for comparisons.

Many of the results from the Helios observations are described in a two volume book: Physics of the Inner Heliosphere (eds. R. Schwenn and E. Marsch, Springer-Verlag, Berlin, 1990). Helios measurements were used to investigate the structure of the undisturbed inner heliosphere (e.g. Rhodes and Smith 1975; Behannon et al. 1989), as well as its disturbed state. Especially relevant to STEREO are the results on the speeds and heliolongitude extents of interplanetary shocks resulting from imaged Solwind CMEs (e.g., Sheeley et al. 1985), and the confirmation of the interplanetary shock origins and heliolongitude distribution of CME-related solar energetic particles (e.g., Beeck et al. 1987). A number of these and other seminal studies were carried out on the Helios data set as recently as the nineties (e.g., Kallenrode 1993, 1995; Reames et al. 1996; Richardson 1994; Bothmer and Schwenn 1998). The radial sampling afforded by the Helios elliptical orbits, reaching inward to within $0.3 \mathrm{AU}$ of the Sun and out to $1 \mathrm{AU}$, provided a further dimension to Helios-based studies of solar wind structure, ICMEs and SEPs that will continue to distinguish these observations, even from those obtained with STEREO.

However, while Sheeley et al. (1985) and Bothmer and Schwenn (1996) made use of the Solwind coronagraph images of CMEs in their studies of Helios interplanetary shock sources and ICME sources, they could only obtain suggestive results on solar causes and interplanetary effects, rather than detailed interpretations connecting specific solar structures with in situ events. The STEREO project has the great advantage of state-of-the-art stereoscopic imaging from SECCHI, of updated analysis capabilities and knowledge of both the solar wind and CMEs/ICMEs from Yohkoh, SOHO, ACE and WIND, and of the involvement of sophisticated, realistic modeling efforts.

Some instrument-specific data analysis tools based on decades of team experience are available at the individual hardware provider sites (see the accompanying papers on SWEA, STE, MAG, SIT, SEPT, LET and HET by Sauvaud et al., Lin et al., Acuna et al., Mason et al., Mewaldt et al., von Rosenvinge et al., Mueller-Mellin et al., all in this volume). In contrast, IMPACT's main data portal is a gateway to the combined investigation designed to take advantage of all of the complementary in situ measurements on STEREO plus connections to the imaging and radio remote sensing results. Our first step toward enabling IMPACT data interpretation beyond the provision of the browse plots and the Level 1 data access and plotting capability (described in the previous section) is the routine merging of key STEREO in situ data in the Level 2 data files described above. Our second step is to provide an easy interface to complementary near-Earth in situ measurements from ACE and WIND to add additional multipoint perspectives and better connect STEREO events to geoeffects. Our third step is to provide links to selected SECCHI and other imaging results and additional complementary information (e.g. archived solar magnetograms and magnetogram-derived synoptic maps). Our ultimate step will be to merge the in situ results with the results from the realistic solar wind and CME/ICME modeling efforts. Toward this end, the Level 3 event and feature lists will provide a useful catalogue from which to select desirable periods.

The ability to analyze in situ space weather events in detail depends on the uninterrupted availability of all of the basic measurements: solar wind plasma density, ion and 
elemental composition, bulk velocity, ion and electron temperatures, the vector magnetic field, and energetic particle fluxes. The set browse plots of Carrington Rotation-size intervals, mentioned earlier, provide a natural starting point for scanning for events and large scale structures. Week-long and daily set plots allow examination of further detail, including transient events. The spacecraft locations are displayed for each plot interval. Although the slowly separating motion of the STEREO spacecraft will generally not matter for the few-day or longer periods of interest in CME case studies, their separation is a critical piece of information. The science quality (as distinguished from Beacon) plasma moments, magnetic fields, and basic SEP data available from IMPACT and PLASTIC as 1-min averages in the Level 2 data are the next natural point of contact. Note that these data, in contrast to their predecessors, include the heat flux $(>100 \mathrm{eV})$ electron angular distributions and ion composition. Counterstreaming heat flux electrons, like low ion temperatures and large magnitude, low variance, smoothly rotating magnetic fields, have become widely accepted diagnostics of ICME ejecta (e.g., Neugebauer and Goldstein 1997; Shodhan et al. 2000). Similarly, the suprathermal electron anisotropy is used to infer the topology of the interplanetary magnetic field, including the field near the heliospheric current sheet (e.g., Kahler et al. 1996; Crooker et al. 1993, 1998). In the past, it was usual to exclude these data from routine displays of interplanetary plasma and field moments in online browsers, but advancements in interactive plotting allow this to be routine for IMPACT data access sites, and SWEA's nearly $4 \pi$ coverage of the heat flux electron distribution allows a more accurate interpretation of the electron anisotropies. Level 1 data can be used for even more detail. Recently Gosling et al. (2006b) searched the multipoint plasma and magnetic field measurements from Helios for signatures of extended magnetic reconnection X-lines in the solar wind. IMPACT data contents and displays make such studies easily possible.

SEPs are considered the number one priority for space weather prediction at NOAA SEC (T. Onsager, NOAA SEC, personal communication, 2002), and are another way to remotely sense events and structures in the corona. On Helios, SEP observation studies made the most use of the multipoint sampling. However, many of these early Helios SEP event analyses did not use the context provided by the solar wind and interplanetary magnetic field observations, with the exception of confirming the timing of shock passages (e.g. Kahler et al. 1984; Beeck et al. 1987). In more recent studies using Helios data (e.g. Reames et al. 1996) the advantage of co-analyzing these measurements is clearly demonstrated. IMPACT data include SEP fluxes, compositions, and anisotropies as part of the in situ merged data sets to enable analyses of their heliospheric context at the two STEREO locations at all times.

It is anticipated that by the end of the STEREO 2-year prime mission in 2009, we will have accumulated a significant sample of well-observed events for analysis. Figure 20 contains information on previous solar cycle frequencies of observed CMEs, ICMEs, and SEP enhancements. As STEREO's prime mission is on the leading edge of the rising phase of cycle 24 , extending observations for only a few years will more than double the available library of events for study.

Near-Earth Data for Comparisons Schwenn et al. (1978), Burlaga et al. (1978) and Reames et al. (1996) demonstrated the value of comparisons between near-Earth data sets and the Helios plasma, field, and SEP observations. The near Earth data offer both an additional multipoint measurement location and ground-truth for the potential geospace effects of an event seen by other spacecraft. The most complementary data sets from ACE and WIND for STEREO comparisons are listed in Table 6. ACE and WIND observations are 

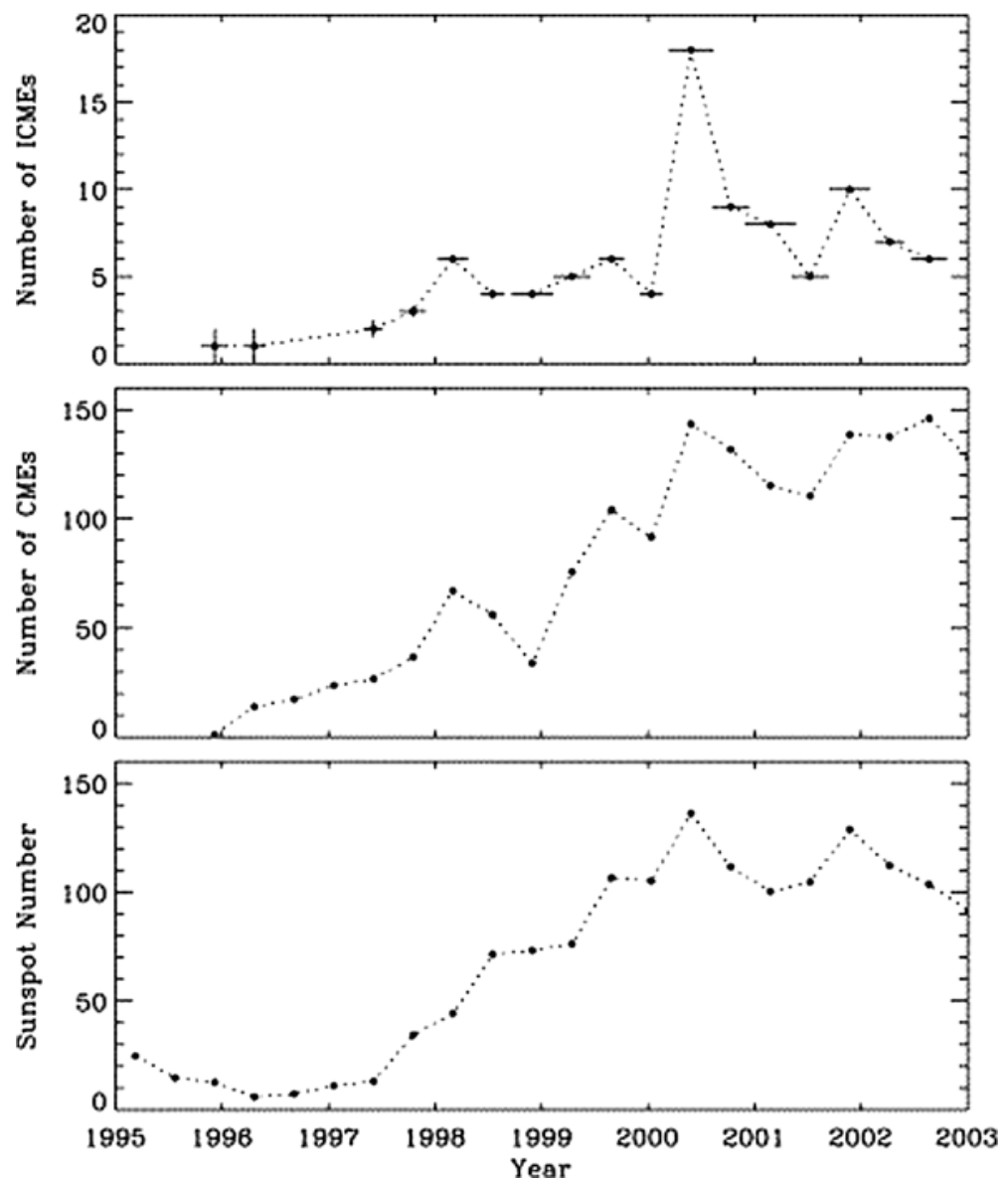

(a)

Fig. 20 a CME and ICME rate survey statistics from Liu et al. (2005) for a large portion of the previous solar cycle. b SEP event statistics (from Mewaldt, personal communication 2005)

available through the IMPACT Level 1 and Level 2 data access sites to provide the in situ Earth-location perspective throughout the STEREO mission. GOES proton data links are provided as a reminder of these complementary magnetospheric measurements which are a measure of the geospace consequences of solar events.

Integration with Coronagraph and Other Imaging Observations As mentioned earlier, Sheeley et al. (1985) and Bothmer and Schwenn (1994) used available coronagraph observations to interpret the solar sources of the Helios in situ events, including shocks, ICMEs and SEPs. The SMM Coronagraph imaged a quadrant of the Sun between 1.6 and 4.1 solar radii, with full Sun images obtained by pasting four sequential quadrant images together (Burkepile and St. Cyr 1993). Unfortunately, SMM experienced a period of nonoperation during the years 1981-mid 1984, losing a large fraction of its potential common observing period with Helios. However, the full-Sun, but lower spatial resolution, Solwind Coronagraph covered the Helios period during the greater part of Cycle 21, including the 


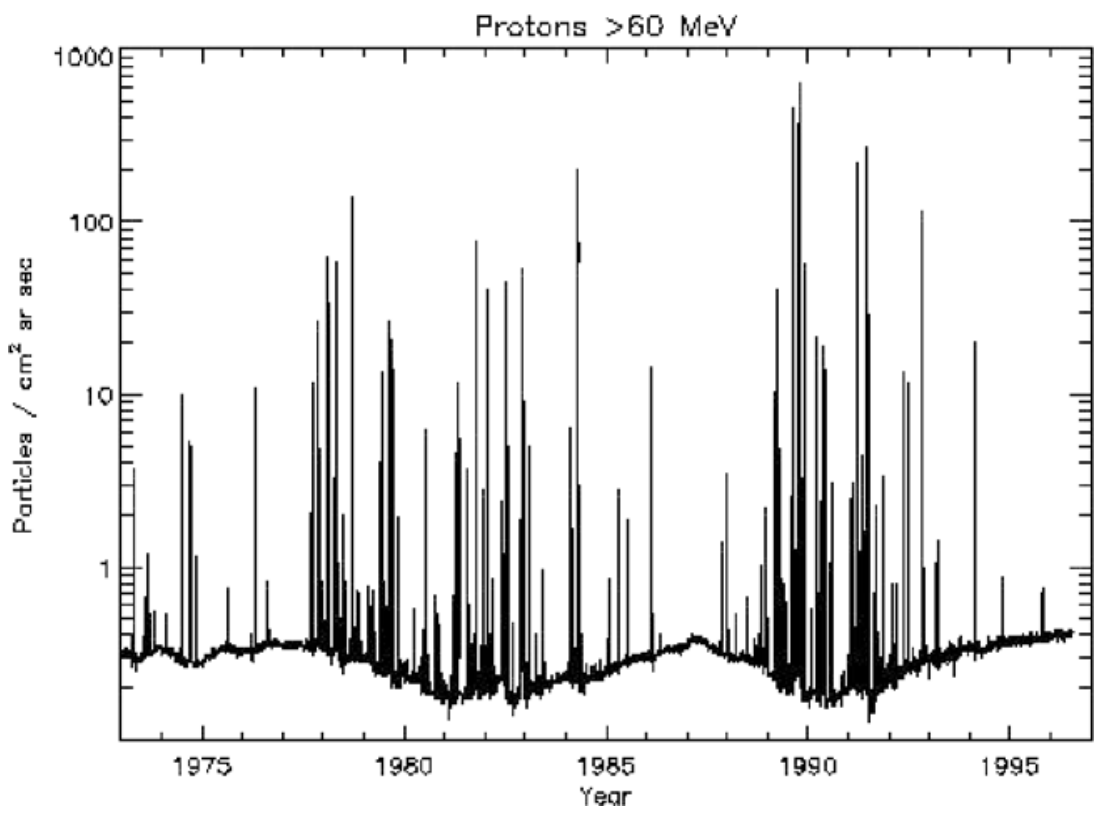

(b)

Fig. 20 (Continued)

Table 6 IMPACT instrument/measurement counterparts on ACE and WIND

\begin{tabular}{llll}
\hline Measurement & IMPACT & ACE & WIND \\
\hline Magnetic field & MAG & MAG & MFI \\
Solar wind plasma & SWEA, PLASTIC & SWEPAM, SWICS & SWE, 3DP \\
Suprathermal e- & SWEA, STE & SWEPAM & SWE, 3DP \\
Suprathermal ions & SIT & ULEIS & EPACT/STEP \\
Energetic electrons & SEPT, HET & EPAM & \\
Energetic ions & SEPT, SIT, & ULEIS, CRIS, & EPACT/STEP \\
& LET, HET & SIS, EPAM & \\
\hline
\end{tabular}

solar maximum which was observed in part by SMM. The state-of-the-art SECCHI data, including SOHO EIT-like EUV images, represent major advances in high-quality, multispectral coronal imaging over the Helios era observations. The IMPACT data access sites take advantage of this by providing links to relevant SECCHI (and SOHO) images. Sites where RHESSI data, TRACE and SXI EUV solar images, and Solar-B soft X-ray images can be obtained are also linked to the IMPACT data server site, with potential for closer integrations and event studies.

Tools for Connecting Multipoint In Situ Data and Solar Data A sample page from our preliminary web interface to the IMPACT browse plots is shown in Fig. 21. Spacecraft orbit locations for the period of the time series plot (here using Helios data) are included at the bottom. These plots are all clickable for magnification and printing or downloading. In 

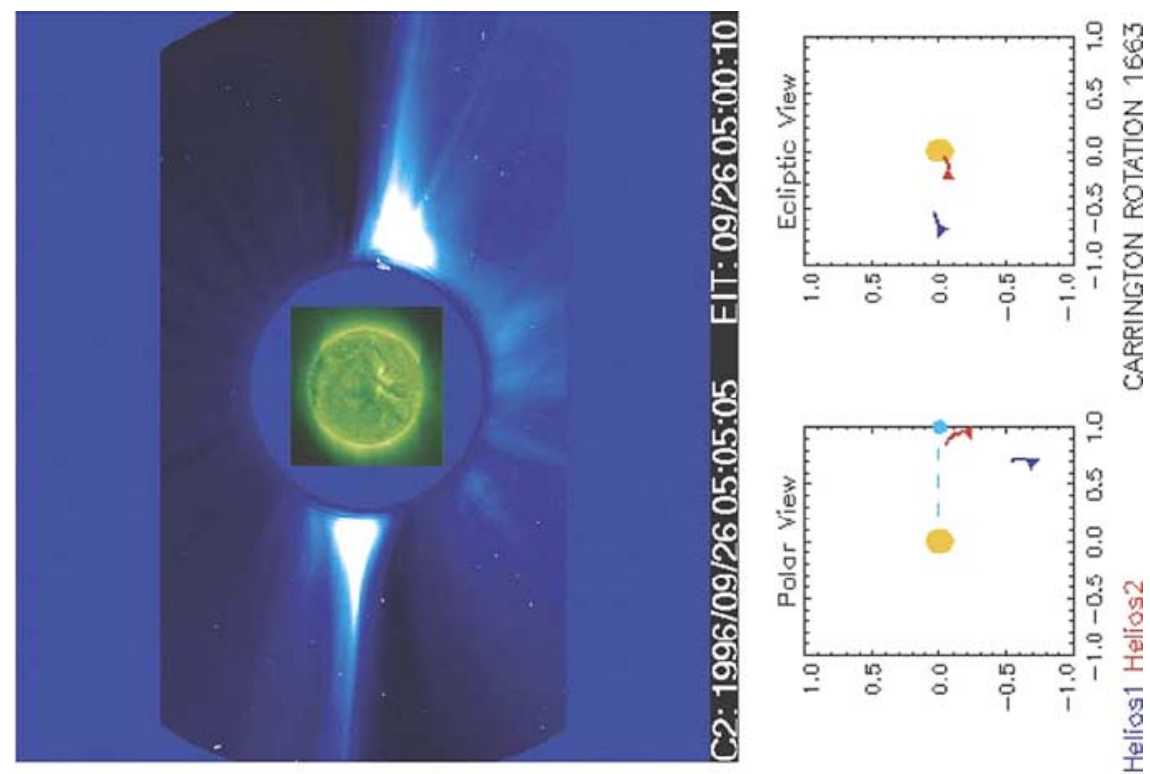

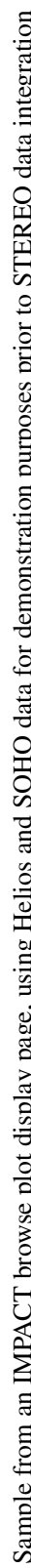

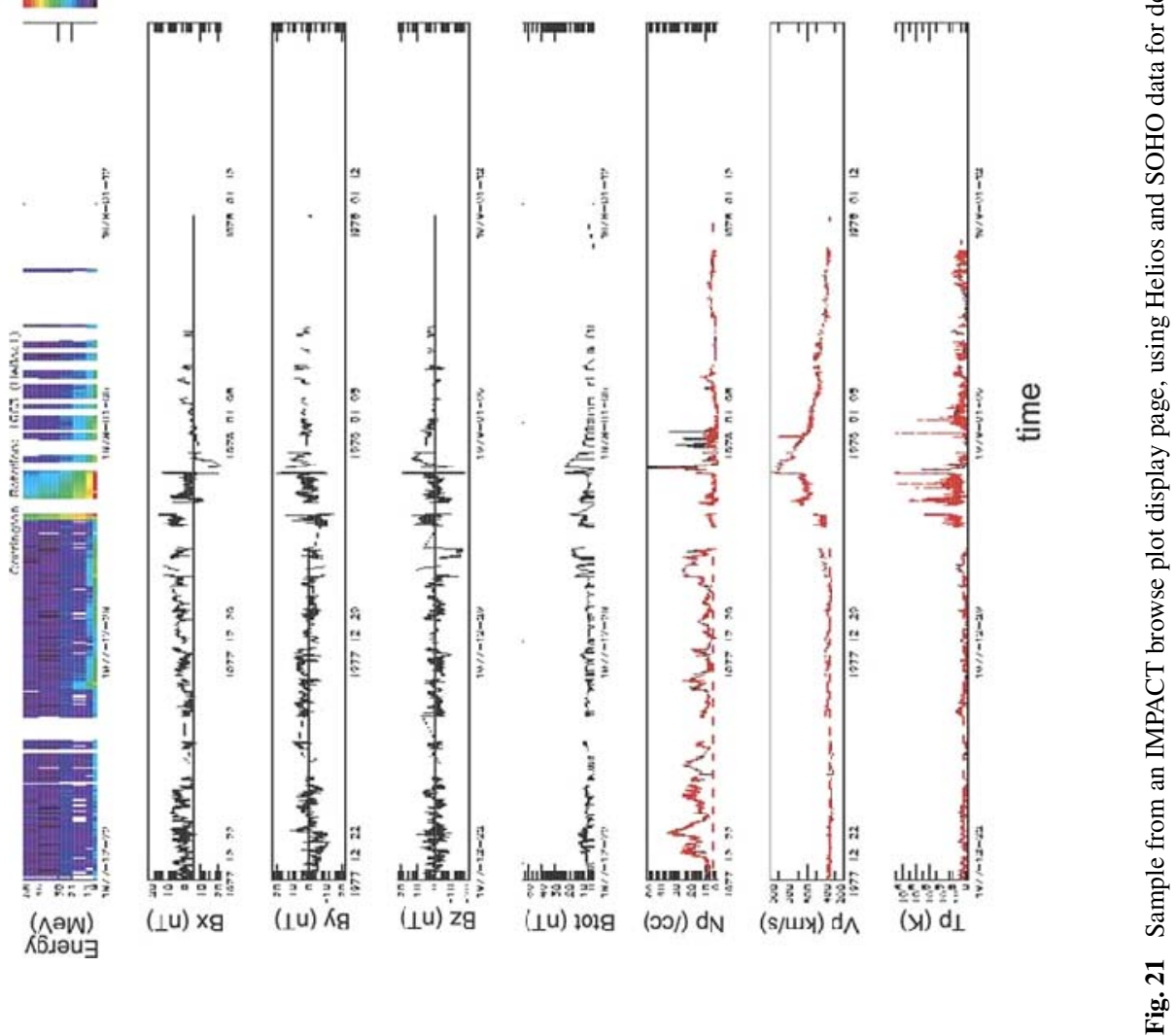

望 Springer 
addition, toggling between displays for the two spacecraft or adjacent Carrington Rotations (CRs) is possible as a means of scanning for features and patterns. Note that we display the SEP data as time vs. energy vs. flux spectrograms as well as time series. Suprathermal electron pitch angle distributions are also included in similar spectrogram form as time vs. angle vs. flux panels. IMPACT's more customizable plot generating tool was described in the previous section on Data Products. That tool also allows timeline data overlays including lags and leads of the timeline for each data set plotted-a useful capability for testing corotation and other timing delays.

Our challenge has been the design of additional displays that allow the solar connection to be made in an insightful way. While it is a simple matter to link the events that correspond to each browse plot interval (allowing for 4-5 days quiet solar wind transit times and minutes to days for transient events), it is not as obvious how to allow the user to tell, for example, where on the Sun the spacecraft map to along the interplanetary field at a given time, and how a mapped field line relates to the inferred location of a CME seen in a coronagraph image.

The standard and well-tested approach for first-order connection of the corona to points in the inner heliosphere is the potential field source surface model of the coronal magnetic field, mapped kinematically or quasi-kinematically outward. A version of such a model developed by Arge and Pizzo (2000), based on Sheeley and Wang's (1991) empirical relationship between coronal open field divergence and solar wind speed, is currently used to predict solar wind sources, solar wind velocities, and interplanetary field polarity at L1 at the NOAA SEC Rapid Prototyping Center (http://solar.sec.noaa.gov/ws/). Figure 22 shows sample products from this model, which is based on synoptic maps of the photospheric field available from a number of ground based observatories. The model can be applied to take

Derived Coronal Holes from National Solar Observatory/SOLIS

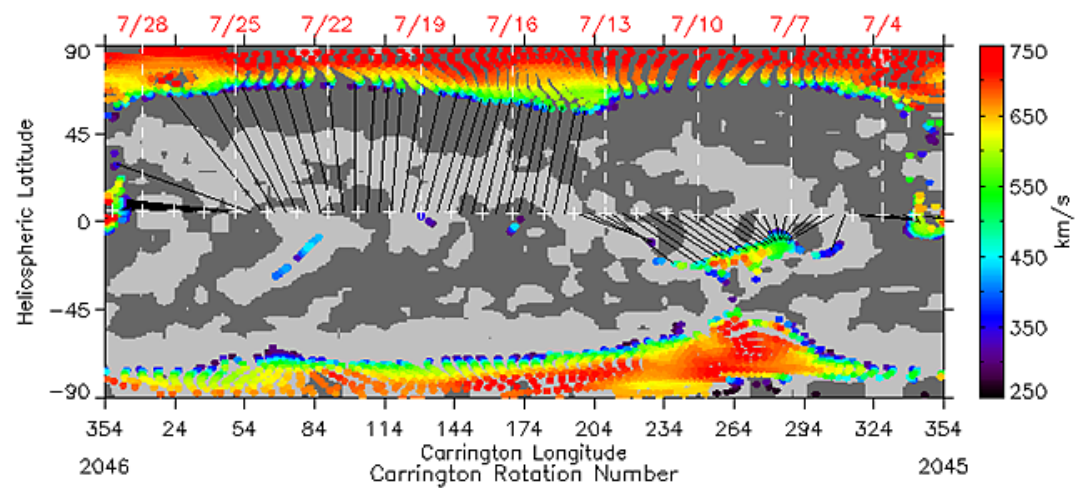

Created 2006 Jul 251625 UTC

NOAA/SEC, BOULDER, CO, USA

(a)

Fig. 22 Sample plots of WSA (Wang-Sheeley-Arge) solar wind model results from the NOAA SEC website. a Coronal hole areas (colored) superposed on a synoptic map of the photospheric magnetic field. The color code indicates the model solar wind velocity arising from a particular open coronal field location. The black lines indicate what maps to the ecliptic plane and thus affects the Earth. b The L1 time series of velocity inferred from the model results in a. The various color points are from models based on the magnetic field maps from different solar observatories. The black line shows the ACE measurements. b The interplanetary magnetic field polarity inferred from the results in $\mathbf{a}$ 


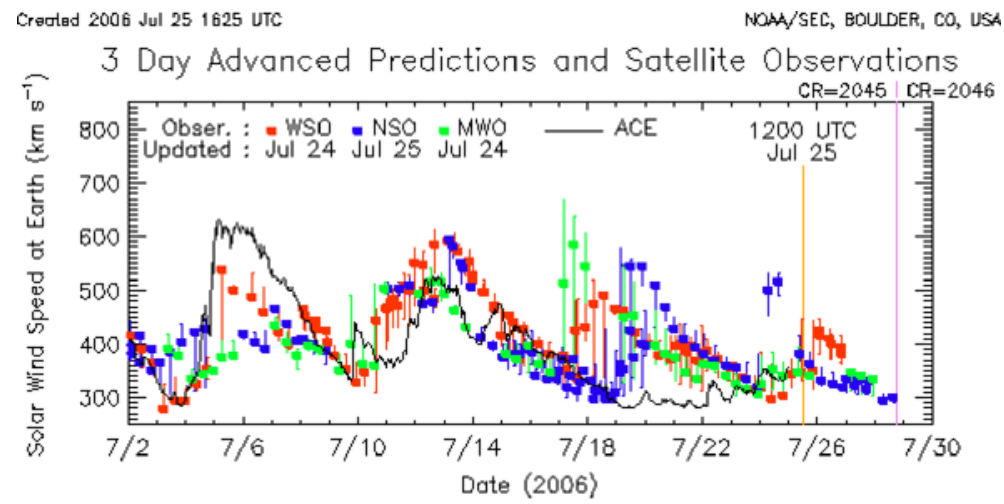

Created 2006 Jul 251621 UTC

NOAA/SEC, BOULDER, CO, USA

(b)

3 Day Advanced Predictions and Satellite Observations

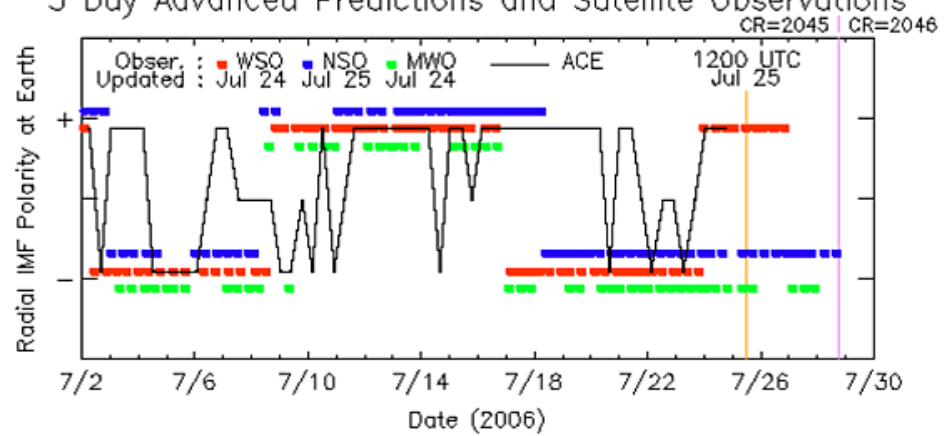

Created 2006 Jul 251621 UTC

NOAA/SEC, BOULDER, CO, USA

Fig. 22 (Continued)

advantage both of distributed in situ measurements for assessing conditions near Earth, and for relating a coronagraph image and EUV maps of coronal holes to the multipoint in situ solar wind and interplanetary magnetic field time series.

Analysis of solar wind structure and transient events The analysis of 3D solar wind structure, especially before solar activity increases toward the next cycle maximum, is greatly enhanced by access to the combination of SECCHI and SOHO EUV images of coronal holes and 3D models of the solar wind structure such as the Wang/Sheeley/Arge (WSA) model discussed above. The WSA model is now available at the CCMC (Community Coordinated Modeling Center) for general use, along with several other IMPACT team coronal and heliospheric models. Routine STEREO spacecraft data comparisons with this model, in parallel with the ACE and WIND in situ data comparisons (of solar wind speed and interplanetary field polarity) are expected to be added to the SEC WSA model displays shown earlier (N. Arge, personal communication). These model applications and others can be used to trace sources of solar wind streams and boundaries with different attributes, such as those displaying counterstreaming or sunward streaming suprathermal electron features, or exhibiting unusual densities, composition, velocities, or magnetic fields seen by IMPACT and PLASTIC. They can also give insight into the STEREO spaceraft magnetic connectivity to the vicinity of active regions. The great advantage of using the models is that they provide 
a physics-based global framework for co-analyzing and integrating the multipoint data sets from STEREO, ACE and WIND (and other available spacecraft).

Armed with the on-disk solar signatures from SECCHI of the coronal morphology of the $\mathrm{CME}$, and the potential field source surface or MHD models of the prevailing large scale coronal magnetic field and related solar wind structure, one can examine the relationship between the involved active region, the overlying larger scale magnetic fields, and the orientation of the fields in the ejecta structure observed at both spacecraft locations. The inward extensions of these models allow us to analyze the spacecraft magnetic connections to the coronal eruption site for the purpose of remotely probing the coronal shock with SEPs. While the possibilities provided by the models are promising, we have not yet developed concepts for their routine application to STEREO transient events.

Special considerations apply to quadrature studies when what is imaged by one SECCHI coronagraph at the limb is detected by the other STEREO spacecraft (or ACE or WIND). The STEREO spacecraft will be 90 degrees apart at the end of the prime mission (see Kaiser et al., this volume, 2007). If an extended mission occurs, each STEREO spacecraft will be in quadrature with ACE, WIND and SOHO in $~ 2,010$, the approximate time of the next solar maximum. Sheeley et al. (1985) created a list of 56 matches of Solwind CMEs and interplanetary shocks observed by either Helios spacecraft when it was within 30 degrees of a limb. They also noted H-alpha flares and GOES X-ray events occurring before the Helios shocks. Bothmer and Schwenn (1996) selected a subset of these when ICME ejecta were also detected, and a smaller subset where solar observations of an erupting polar crown filament preceded detection of a magnetic cloud flux rope. Similar event and feature tables are being collected for STEREO as part of the SSC activities (Thompson et al., this volume, 2007) and IMPACT Level 3 data sets (see previous section).

Development of potential operational schemes One of the outstanding questions for STEREO mission planning is how best to use in situ multipoint measurements to enhance space weather forecasting capabilities. The most obvious application is the monitoring of corotating solar wind structure on the Behind spacecraft that trails Earth in its orbit structure that may survive until that part of the solar wind corotates to Earth (e.g., Burlaga et al. 1987). Toward this application, and also as a test of solar wind structure steadiness, and hence predictability, we provide for time-shifted time series plots at both IMPACT Level 1 and Level 2 data server sites. The WSA model described above will provide a useful framework for organizing and interpreting corotated and translated features.

Another possible application of the IMPACT data is the deduction from its multipoint SEP measurements of the likelihood that the ICME shock nose will either magnetically connect to or impact Earth, implying maximum SEP fluxes and a storm sudden commencement with its associated geospace effects. Reames et al. (1996) showed that SEP time profiles at spaced locations in the heliosphere follow a pattern that depends on relative shock nose location. Observation-based versions of their classic picture, reproduced in Fig. 4, can be used to qualitatively estimate the SEP and storm impact of events at Earth from what is seen at the STEREO locations. We also use corotational shifts to monitor whether SEP events observed on the Behind spacecraft, like solar wind structure, provide some prediction of SEP behavior when that particular interplanetary flux channel connects to Earth. As SEP events are typically several days long, this may prove most useful for separations of $\sim 30 \mathrm{deg}$ or less. On the other hand, the prompt SEPs observed at the Behind spacecraft when the separation is greater may provide warning of the approach to Earth of a strong shock with its associated ESP increase, given the fact that the spiral interplanetary field lines threading it sample the corona at low altitude in the subsolar region. 
Finally, applications of IMPACT data to studies of the solar wind interactions with the planets are enabled both by plots of the STEREO spacecraft locations relative to the planets available at SSC (Thompson et al.), and merged SWEA-type data sets from Mars Express and Venus Express available at the IMPACT SWEA site at CESR.

\subsection{Expected Impact and Relevance to Heliophysics}

The STEREO mission was initiated explicitly for improving our understanding of the origin, propagation, and $1 \mathrm{AU}$ consequences of CMEs, as well as the origins of other solar wind structures.

As a result of the IMPACT investigation, a new, more comprehensive $1 \mathrm{AU}$ in situ data set is available for several heliolongitudes. In particular, it includes the information on suprathermal electron angular distributions often used in the analysis of solar wind structure and CME/ICME events, together with solar wind electron, magnetic field and SEP data. Coordinations with PLASTIC and SWAVES provide subsets of the complete STEREO in situ data on a routine basis. The corresponding near-Earth data from ACE and WIND are moreover made easily accessible through IMPACT data access sites for coordinated analyses. Complementary coronagraph images from SECCHI and SOHO, and solar magnetograph information important for mapping the interplanetary medium to the Sun, are also linked from the IMPACT data access site and displayed in ways that encourage analyses of the physical connections. This resource is made available to the heliophysics community for the lifetime of the STEREO mission and is also integrated into the developing Virtual Heliospheric Observatory for long-term exploitation. STEREO is the testbed for future Heliospheric Constellations as well as space weather monitoring missions. Should it survive its 2013-2015 transit of the farside of the Sun, IMPACT can again become an active participant in providing widespread heliospheric observations for the new rising phase of solar cycle 25. Coordinations with SDO and ESA's Solar Orbiter missions, and a supporting role for any ongoing human exploration activities, will be among its future objectives and goals.

Acknowledgements The IMPACT team is grateful for the support provided by many colleagues in many capacities, including: M. Mandell, G. Ginet, J.B. Blake, J. Fennell, H. Maldonado, H. Culver, A. Harper, M. Delmont, N. Chrissotimos, E. Reynolds, A. Driesman, W. Thompson, E. Christian, B. Anderson, M. Kaiser, R. Jackson, S. McBride, M. Colby, P. Berg, S. Heavner, R. Ullrich (Boom Designer), P. Turin, K. Harps, P. Dobson, S. Lam, M. Hashii, H. Yuan, C. Ingraham, I. Sircar, L. Guhathakurta, R. Fisher. IMPACT is supported by NASA contract NAS5-00133.

\section{References}

Acuna et al. (this volume, 2007)

S.K. Antiochos, C.R. DeVore, J.A. Klimchuk, Astrophys. J. 510, 485 (1999)

C.N. Arge, V. Pizzo, J. Geophys. Res. 105, 10465 (2000)

Aschwanden et al. (this volume, 2006). doi: 10.1007/s11214-006-9027-8

C.C. Balch, Radiat. Meas. 30, 231 (1999)

S.D. Bale, P.J. Kellogg, D.E. Larson, R.P. Lin, K. Goetz, R.P. Lepping, Geophys. Res. Lett. 25, 2929 (1998a)

S.D. Bale, P.J. Kellogg, K. Goetz, S.J. Monson, Geophys. Res. Lett. 25, 9 (1998b)

S.D. Bale, M.J. Reiner, J.-L. Bougeret, M.L. Kaiser, S. Krucker, D.E. Larson, R.P. Lin, Geophys. Res. Lett. 26, 1573 (1999)

S.J. Bame, J.R. Asbridge, W.C. Feldman, E.E. Fenimore, J.T. Gosling, Solar Phys. 62, 179 (1979)

J. Beeck et al., Astrophys. J. 322, 1052 (1987)

K.W. Behannon, L.F. Burlaga, J.T. Hoeksema, L.W. Klein, J. Geophys. Res. 94, 1245 (1989)

Biesecker et al. (this volume, 2007). doi: 10.1007/s11214-007-9165-7

V. Bothmer, R. Schwenn, Space Sci. Rev. 70, 215 (1994) 
V. Bothmer, D.M. Rust, in Coronal Mass Ejections, ed. by N. Crooker, J. Joselyn, J. Feynman (AGU, 1997), p. 139

V. Bothmer, R. Schwenn, Adv. Space Res. 17, 322 (1996)

V. Bothmer, R. Schwenn, Annales Geophysicae 16, 1 (1998)

Bougeret et al. (this volume, 2007)

H.H. Breneman, E.C. Stone, Astrophys. J. Lett. 299, L57 (1985)

J.T. Burkepile, O.C. St. Cyr, A revised and expanded catalogue of mass ejections observed by the solar maximum mission coronagraph. NCAR Tech. Note NCAR/TN-369+STR, January 1993

L.F. Burlaga et al., J. Geophys. Res. 83, 5167 (1978)

L.F. Burlaga, K.W. Behannon, L.W. Klein, J. Geophys. Res. 92, 5725 (1987)

H.V. Cane, Space Sci. Rev. 93, 55 (2000)

H.V. Cane, D.V. Reames, T.T. Rosenvinge, J. Geophys. Res. 93, 9555 (1988)

H.V. Cane, T.T. von Rosenvinge, C.M.S. Cohen, R.A. Mewaldt, Geophys. Res. Lett. 30 (2003). doi:2002GL016580

R.C. Canfield, H.S. Hudson, D.E. McKenzie, Geophys. Res. Lett. 26, 627-630 (1999)

P.J. Cargill, J. Chen, D.S. Spicer, S.T. Zalesak, J. Geophys. Res. 101, 4855 (1996)

N.U. Crooker, G.L. Siscoe, S. Shodhan, D.F. Webb, J.T. Gosling, E.J. Smith, J. Geophys. Res. 98, 9371 (1993)

N.U. Crooker, D.S. Intriligator, J. Geophys. Res. 101, 24343 (1996)

N.U. Crooker, A.H. McAllister, R.J. Fitzenreiter, J.A. Linker, D.E. Larson, R.P. Lepping, A. Szabo, J.T. Steinberg, A.J. Lazarus, Z. Mikic, R.P. Lin, J. Geophys. Res. 103, 26,859 (1998)

M.I. Desai, G.M. Mason, J.E. Mazur, J.R. Dwyer, Astrophys. J. 645, L81 (2006)

T. Detman, J. Joselyn, in Solar Wind Nine, ed. by S. Habbal, R. Esser, J. Hollweg, P. Isenburg. AIP Conference Proceedings, vol. 471 (1999), p. 729

J.R. Dwyer, G.M. Mason, J.E. Mazur, J.R. Jokipii, T.T. von Rosenvinge, R.P. Lepping, Astrophys. J. (Lett.) 490, L115 (1997)

W.C. Feldman et al., Phys. Rev. Lett. 49, 199 (1982)

J. Feynman, A.J. Hundhausen, J. Geophys. Res. 99, 8451 (1994)

J. Feynman, S.F. Martin, J. Geophys. Res. 100, 3355 (1995)

L.A. Fisk, B. Kozlovsky, R. Ramaty, Astrophys. J. 190, L35 (1974)

N.J. Fox, M. Peredo, B.J. Thompson, Geophys. Res. Lett. 25, 2461 (1998)

H.O. Funsten, J.T. Gosling, P. Riley, O.C. St. Cyr, R. Forsyth, R.A. Howard, R. Schwenn, J. Geophys. Res. 104, 6679 (1999)

Galvin et al. (this volume, 2007)

T.L. Garrard, A.J. Davis, J.S. Hammond, S.R. Sears, Space Sci. Rev. 86, 649 (1998)

M. Gedalin, Geophys. Res. Lett. 26, 1239 (1999)

S.E. Gibson, B.C. Low, Astrophys. J. 493, 460 (1998)

G. Gloeckler, L.A. Fisk, S. Hefti, N.A. Schwadron, T.H. Zurbuchen, F.M. Ipavich, J. Geiss, P. Bochsler, R.F. Wimmer-Schweingruber, Geophys. Res. Lett. 26, 157 (1999)

C.C. Goodrich, J.D. Scudder, J. Geophys. Res. 89, 6654 (1984)

N. Gopalswamy, S. Yashiro, G. Michalek, M.L. Kaiser, R.A. Howard, R. Leske, T. von Rosenvinge, D.V. Reames, in Solar Wind Ten, ed. by M. Velli, R. Bruno, F. Malara, Wind Conference, AIP Conference Proceedings, vol. 679 (2003), pp. 608

J.T. Gosling, E. Hildner, R.M. MacQueen, R.H. Munro, A.I. Poland, C.L. Ross, Solar Phys. 48, 389 (1976)

J.T. Gosling, J.R. Asbridge, S.J. Bame, W.C. Feldman, R.D. Zwickl, J. Geophys. Res. 85, 3431 (1980)

J.T. Gosling, D.N. Baker, S.J. Bame, W.C. Feldman, R.D. Zwickl, E.J. Smith, J. Geophys. Res. 92, 8519 (1987)

J.T. Gosling, D.J. McComas, Geophys. Res. Lett. 14, 355 (1987)

J.T. Gosling, in Physics of Magnetic Flux Ropes, ed. by C.T. Russell, E.R. Priest, L.C. Lee, Geophysical Monograph, vol. 58 (AGU, 1990), pp. 343-364

J.T. Gosling, S.J. Bame, D.J. McComas, J.L. Phillips, Geophys. Res. Lett. 17, 901 (1990)

J.T. Gosling, D.J. McComas, J.L. Phillips, S.J. Bame, J. Geophys. Res. 96, 7831 (1991)

J.T. Gosling, D.J. McComas, J.L. Phillips, S.J. Bame, J. Geophys. Res. 97, 6531 (1992)

J.T. Gosling, J. Geophys. Res. 98, 18,937-18,949 (1993)

J.T. Gosling, S.J. Bame, D.J. McComas, J.L. Phillips, B.E. Goldstein, M. Neugebauer, Geophys. Res. Lett. 21, 1105 (1994a)

J.T. Gosling, D.J. McComas, J.L. Phillips, L.A. Weiss, V.J. Pizzo, B.E. Goldstein, R.J. Forsyth, Geophys. Res. Lett. 21, 2271 (1994b)

J.T. Gosling, J. Birn, M. Hesse, Geophys. Res. Lett. 22, 869-872 (1995a)

J.T. Gosling, D.J. McComas, J.L. Phillips, V.J. Pizzo, B.E. Goldstein, R.J. Forsyth, R.P. Lepping, Geophys. Res. Lett. 22, 1753 (1995b) 
J.T. Gosling, Annu. Rev. Astron. Astrophys. 34, 35 (1996)

J.T. Gosling, P. Riley, Geophys. Res. Lett. 23, 2867-2870 (1996)

J.T. Gosling, in Coronal Mass Ejections, ed. by N.U. Crooker, J.A. Joselyn, J. Feynman, Geophysical Monograph, vol. 99 (AGU, 1997a), pp. 9-16

J.T. Gosling, in Robotic Exploration Close to the Sun: Scientific Basis, ed. by S. Habbal, AIP Conf. Proc., vol. 385 (Woodbury, New York, 1997b), p. 17

J.T. Gosling, V.J. Pizzo, Space Sci. Rev. 89, 21 (1999)

J.T. Gosling, R.M. Skoug, D.J. McComas, C.W. Smith, J. Geophys. Res. 110, A01107 (2005a). doi:10.1029/2004JA010809

J.T. Gosling, R.M. Skoug, D.J. McComas, C.W. Smith, Geophys. Res. Lett. 32, L05105 (2005b). doi:1029/2005GL022406

J.T. Gosling, S. Eriksson, R.M. Skoug, D.J. McComas, R.J. Forsyth, Astrophys. J. 644, 613 (2006a)

J.T. Gosling, S. Erikkson, R. Schwenn, J. Geophys. Res. 111, A10102 (2006b). doi: 10.1029/2006JA011863

D.K. Haggerty, E.C. Roelof, Astrophys. J. 579, 841 (2002)

R.A. Harrison, E. Hildner, A.J. Hundhausen, D.G. Sime, G.M. Simnett, J. Geophys. Res. 95,917 (1990)

Howard et al. (this volume, 2007)

A.J. Hundhausen, in Solar Wind, ed. by C.P. Sonnett, P.J. Coleman, J.M. Wilcox, NASA SP-308 (Washington, 1972), p. 393

A.J. Hundhausen, J. Geophys. Res. 98, 13,177 (1993)

A.J. Hundhausen, J. Burkepile, O.C. St. Cyr, J. Geophys. Res. 99, 6543 (1994)

B.V. Jackson et al., Solar Phys. 225, 177 (2004)

S.W. Kahler, D.V. Reames, J. Geophys. Res. 96, 9419 (1991)

S.W. Kahler et al., J. Geophys. Res. 89, 9683 (1984)

S.W. Kahler, in Proc. of the Third SOHO Workshop, ESA SP-373, December 1994, p. 253

S.W. Kahler, N.U. Crooker, J.T. Gosling, J. Geophys. Res. 101, 24373-24382 (1996)

S.W. Kahler, in Space Weather, ed. by P. Song et al., AGU Monograph, vol. 125 (American Geophysical Union, 2001), p. 109

Kaiser et al. (this volume, 2007)

M.-B. Kallenrode, Adv. Space Res. 13, 341 (1993)

M.-B. Kallenrode, Adv. Space Res. 15, 375 (1995)

P.J. Kellogg, K. Goetz, S.J. Monson, S.D. Bale, J. Geophys. Res. 104, 17069 (1999)

M.G. Kivelson, C.T. Russell, Introduction to Space Physics (Cambridge University Press, Cambridge, 1995)

K.-L. Klein, S. Krucker, G. Tottet, S. Hoang, Astron. Astrophys. 1047 (2005)

A.S. Krieger, A.F. Timothy, E.C. Roelof, Solar Phys. 29, 505 (1973)

S. Krucker, D.E. Larson, R.P. Lin, B.J. Thompson, Astrophys. J. 519, 864 (1999)

A. Kumar, D. Rust, J. Geophys. Res. 101, 15667 (1996)

D. Lario, B. Sanahuja, A.M. Heras, Astrophys. J. 509, 415 (1998)

D.E. Larson et al., Geophys. Res. Lett. 24, 1911 (1997)

D. Larson, R.P. Lin, J. Steinberg, Geophys. Res. Lett. 27, 157 (2000)

R.J. Leamon, R.C. Canfield, S.L. Jones, K. Lambkin, B.J. Lundberg, A.A. Pevstov, J. Geophys. Res. 109 (2004). doi:2003JA010324

M.A. Lee, J. Geophys. Res. 88, 6109 (1983)

M.A. Lee, Astrophys. J. (Suppl.) 158, 38 (2005)

R.P. Lepping et al., J. Geophys. Res. 95, 11957 (1990)

S. Lepri, T. Zurbuchen, L. Fisk, I. Richardson, H. Cane, G. Gloeckler, J. Geophys. Res. 106, 29231 (2001)

G. Li, G.P. Zank, W.K.M. Rice, J. Geophys. Res. 108 (2003). doi:2002JA009666

Y. Li, J. Luhmann, J. Atmos. Terr. Phys. 66, 323 (2004)

Y. Li, J.G. Luhmann, Astrophys. J. 648, 732 (2006)

Y. Li, J.G. Luhmann, T. Mulligan, J.T. Hoeksema, C.N. Arge, S.P. Plunkett, O.C. St. Cyr, J. Geophys. Res. 106, 25,103 (2001)

R.P. Lin, S.W. Kahler, J. Geophys. Res. 97, 8203 (1992)

Lin et al. (this volume, 2007)

G.M. Lindsay, C.T. Russell, J.G. Luhmann, P. Gazis, J. Geophys. Res. 99, 11-17 (1994)

G.M. Lindsay, C.T. Russell, J.G. Luhmann, J. Geophys. Res. 100, 16999-17013 (1995)

G.M. Lindsay, J.G. Luhmann, C.T. Russell, J.T. Gosling, J. Geophys. Res. 104, 12,515 (1999a)

G.M. Lindsay, C.T. Russell, J.G. Luhmann, J. Geophys. Res. 104, 10335-10344 (1999b)

J.A. Linker, Z. Mikic, Astrophys. J. 438, L45 (1995)

Y. Liu, J.D. Richardson, Planet. Space Sci. 53, 3 (2005)

Y. Liu, J.D. Richardson, J.W. Belcher, Planet. Space Sci. 53, 3-17 (2005)

J.G. Luhmann, in Coronal Mass Ejections, Geophysical Monograph, vol. 99 (American Geophysical Union, 1997), pp. 291-299 
J.G. Luhmann, J.T. Gosling, J.T. Hoeksema, X. Zhao, J. Geophys. Res. 103, 6585-6593 (1998)

J.G. Luhmann, Y.Li, C.N. Arge, P.R. Gazis, R. Ulrich, J. Geophys. Res. 107 (2002). doi:2001JA007550

J.G. Luhmann et al., Adv. Space Res. 36, 1534 (2005)

W.B. Manchester, T.I. Gombosi, D.L. De Zeeuw, I.V. Sokolov, I.I. Roussev, K.G. Powell, J. Kóta, G. Tóth, T.H. Zurbuchen, Astrophys. J. 622, 1225 (2005)

G.M. Mason, J.E. Mazur, J.R. Dwyer, D.V. Reames, T.T. von Rosenvinge, Astrophys. J. Lett. 486, L149 (1997)

Mason et al. (this volume, 2007). doi: 10.1007/s11214-006-9087-9

J.E. Mazur, G.M. Mason, J.R. Dwyer, T.T. von Rosenvinge, Geophys. Res. Lett. 25, 2521 (1998)

D.J. McComas et al., J. Geophys. Res. 94, 6907 (1989)

D.J. McComas et al., J. Geophys. Res. 97, 171 (1992)

D.J. McComas, Rev. Geophys., Suppl. 603 (1995)

D.J. McComas, in Solar Wind Ten, ed. by M. Velli, R. Bruno, F. Malara. AIP Conference Proceedings, vol. 679 (2003), p. 33

D.J. McComas, H.A. Elliott, N.A. Schwadron, J.T. Gosling, R.M. Skoug, B.E. Goldstein, Geophys. Res. Lett. 301910 (2003). doi:10.1029.2003GL017135

Mewaldt et al. (this volume, 2007)

M.D. Montgomery, J.R. Asbridge, S.J. Bame, W.C. Feldman, J. Geophys. Res. 79, 3103 (1974)

Mueller-Mellin et al. (this volume, 2007). doi: 10.1007/s11214-007-9204-4

T. Mulligan, C.T. Russell, J.G. Luhmann, Geophys. Res. Lett. 25, 2959-2962 (1998)

T. Mulligan, C.T. Russell, J. Geophys. Res., 10,581 (2001)

M. Neugebauer, R. Goldstein, in Coronal Mass Ejections, ed. by N.U. Crooker, J.A. Joselyn, J. Feynman. Geophysical Monograph, vol. 99 (AGU, 1997), pp. 245-251

M. Neugebauer et al., J. Geophys. Res. 103, 14,587 (1998)

C.K. Ng, D.V. Reames, A.J. Tylka, Geophys. Res. Lett. 26, 2145-2148 (1999)

D. Odstrcil, V.J. Pizzo, J. Geophys. Res. 104, 28,225 (1999a)

D. Odstrcil, V.J. Pizzo, J. Geophys. Res. 104, 483 (1999b)

D. Odstrcil, V.J. Pizzo, J. Geophys. Res. 104, 493 (1999c)

D. Odstrcil, J.A. Linker, R. Lionello, Z. Mikic, P. Riley, V.J. Pizzo, J.G. Luhmann, J. Geophys. Res. 107 (2002). doi:10.1029/2002JA009334

K.O. Ogilvie, L.F. Burlaga, D.J. Chornay, R.J. Fitzenreiter, J. Geophys. Res. 104, 22389 (1999)

V. Osherovich, L.F. Burlaga, in Coronal Mass Ejections, ed. by N. Crooker, J.A. Joselyn, J. Feynman. Geophysical Monogr. Ser., vol. 99 (AGU, 1997), pp. 157-168

K.I. Paularena, J.D. Richardson, G.N. Zastenker, P.A. Dalin, in Solar Wind Nine, ed. by S. Habbal, R. Esser, J. Hollweg, P. Isenberg (AIP, 1999), p. 585

Phan et al., Nature 439, 175 (2006)

Phillips et al., Geophys. Res. Lett. 22, 3301 (1995)

W.G. Pilipp, H. Miggenrieder, K.-H. Muhlhauser, H. Rosenbauer, R. Schwenn, F.M. Neubauer, J. Geophys. Res. 92, 1103 (1987)

V.J. Pizzo et al., Solar Phys. 226, 283 (2005)

M. Popecki, E. Moebius, B. Klecker, A.B. Galvin, L.M. Kistler, A.T. Bogdanov, in Acceleration and Transport of Energetic Particles Observed in the Heliosphere, ed. by R.A. Mewaldt, J.R. Jokipii, M.A. Lee, E. Moebius, T.H. Zurbuchen. AIP Conference Proceedings, vol. 528 (American Inst. of Physics, New York 2000), pp. 139-142

D.V. Reames, L.M. Barbier, C.K. Ng, Astrophys. J. 466, 473 (1996)

D.V. Reames, S.W. Kahler, C.K. Ng, Astrophys. J. 491, 414 (1997)

D.V. Reames, C.K. Ng, Astrophys. J. 504, 1002 (1998)

D.V. Reames, Space Sci. Rev. 90, 413 (1999)

D.J. Rhodes Jr., E.J. Smith, J. Geophys. Res. 80, 917 (1975)

I.G. Richardson, Astrophys. J. 420, 926 (1994)

J.D. Richardson, K.I. Paularena, Geophys. Res. Lett. 25, 2097 (1998)

J.D. Richardson, F. Dashevskiy, K.I. Paularena, J. Geophys. Res. 103, 14619 (1998)

P. Riley, J.T. Gosling, V.J. Pizzo, J. Geophys. Res. 102, 14,677 (1997)

P. Riley, J.A. Linker, Z. Mikic, D. Odstrcil, T.H. Zurbuchen, D. Lario, R.P. Lepping, J. Geophys. Res. 108 (2003). doi:10.1029/2002JA009760

I. Roth, M. Temerin, Astrophys. J. 477, 940 (1997)

I. Roussev, T.I. Gombosi, I.V. Sokolov, M. Velli, W. Manchester, DeZeeuw, P. Liewer, G. Toth, J.G. Luhmann, Astrophys. J. 595, L57 (2003)

D.M. Rust, Geophys. Res. Lett. 21, 241 (1994)

T.R. Sanderson, R. Reinhard, P. Van Nes, K.-P. Wenzel, E.J. Smith, B.T. Tsurutani, J. Geophys. Res. 90, 973 (1985) 
Sauvaud et al. (this volume, 2007). doi: 10.1007/s11214-007-9174-6

C.J. Schrijver, K. Harvey, Solar Phys. 150, 1 (1994)

R. Schwenn, M. Montgomery, H. Rosenbauer, H. Miggenrieder, K.H. Muhlhauser, S.J. Bame, W.C. Feldman,

R.T. Hansen, J. Geophys. Res. 83, 1011 (1978)

R. Schwenn, H. Rosenbauer, K.-H. Muhlhauser, Geophys. Res. Lett. 7, 201 (1980)

M.A. Shea, D. Smart, Adv. Space Res. 29, 325 (2002)

N.R. Sheeley Jr., et al., J. Geophys. Res. 90, 163 (1985)

N.R. Sheeley, Y.M. Wang, Solar Phys. 131, 165 (1991)

N.R. Sheeley Jr., et al., Astrophys. J. 484, 472-478 (1997)

S. Shodhan et al., J. Geophys. Res. 105, 27,261 (2000)

R.M. Skoug et al., Geophys. Res. Lett. 26, 161 (1999)

Stone et al., Space Sci. Rev. 86, 357 (1998a)

Stone et al., Space Sci. Rev. 86, 285 (1998b)

B.J. Thompson, E.W. Cliver, N. Nitta, C. Delannée, J.-P. Delaboudinière, Geophys. Res. Lett. 27, 1431 (2000)

Thompson et al. (this volume, 2007)

B.T. Tsurutani, Y.T. Lee, W.D. Gonzalez, F. Tang, Geophys. Res. Lett. 19, 73 (1992)

A.J. Tylka, D.V. Reames, C.K. Ng, Geophys. Res. Lett. 26, 2141 (1999)

Ullrich et al. (this volume, 2007)

T.T. von Rosenvinge, L.M. Barbier, J. Karsch, R. Liberman, M.P. Madden, T. Nolan, D.V. Reames, L. Ryan, S. Singh, H. Trexel, G. Winkert, G.M. Mason, D.C. Hamilton, P.H. Walpole, Space Sci. Rev. 71, 155-206 (1995)

von Rosenvinge et al. (this volume, 2007)

Y.-M. Wang Jr., N.R. Sheeley, Astrophys. J. 365, 372 (1990a)

Y.-M. Wang, N.R. Sheeley, Astrophys. J. 355, 726 (1990b)

Y.M. Wang et al., Astrophys. J. 498, L165-L168 (1998)

D.F. Webb, R.A. Howard, J. Geophys. Res. 99, 4201 (1994)

D.F. Webb, E.W. Cliver, N.U. Crooker, O.C. St. Cyr, B.J. Thompson, J. Geophys. Res. 105, 7491 (2000)

S. Yashiro, N. Gopalswamy, S. Akiyama, G. Michalek, R.A. Howard, J. Geophys. Res. 110 (2005). doi:10.1029/2005JA011151

C.J. Zeitlin, T. Cleghorn, F. Cuccinota, P. Saganti, V. Anderson, K. Lee, L. Pinsky, W. Atwell, R. Turner, G. Badhwar, Adv. Space Res. 33, 2204 (2004)

R.D. Zwickl, K.A. Doggett, S. Sahm, W.P. Barrett, R.N. Grubb, T.R. Detman, V.J. Raben, C.W. Smith, P. Riley, R.E. Gold, R.A. Mewaldt, T. Maruyama, Space Sci. Rev. 86, 633 (1998) 